Noam Berger

\title{
Slowdown estimates for ballistic random walk in random environment
}

Received March 1, 2009 and in revised form February 24, 2010

\begin{abstract}
We consider models of random walk in uniformly elliptic i.i.d. random environment in dimension greater than or equal to 4 , satisfying a condition slightly weaker than the ballisticity condition $\left(T^{\prime}\right)$. We show that for every $\epsilon>0$ and $n$ large enough, the annealed probability of linear slowdown is bounded from above by $\exp \left(-(\log n)^{d-\epsilon}\right)$. This bound almost matches the known lower bound of $\exp \left(-C(\log n)^{d}\right)$, and significantly improves previously known upper bounds. As a corollary we provide almost sharp estimates for the quenched probability of slowdown. As a tool, we show an almost local version of the quenched central limit theorem under the assumption of the same condition.
\end{abstract}

\section{Introduction}

\subsection{Background}

Let $d \geq 1$. A Random Walk in Random Environment (RWRE) on $\mathbb{Z}^{d}$ is defined as follows: Let $\mathcal{M}^{d}$ denote the space of all probability measures on $\left\{ \pm e_{i}\right\}_{i=1}^{d}$ and let $\Omega=$ $\left(\mathcal{M}^{d}\right)^{\mathbb{Z}^{d}}$. An environment is a point $\omega \in \Omega$. Let $P$ be a probability measure on $\Omega$. For the purposes of this paper, we assume that $P$ is an i.i.d. measure, i.e.

$$
P=Q^{\mathbb{Z}^{d}}
$$

for some distribution $Q$ on $\mathcal{M}^{d}$, and that $P$ is uniformly elliptic, i.e. there exist $\eta>0$ such that for every neighbor $v$ of the origin,

$$
Q(\{\omega: \omega(v)<\eta\})=0 .
$$

For an environment $\omega \in \Omega$, the Random Walk on $\omega$ is a time-homogeneous Markov chain with transition kernel

$$
P_{\omega}\left(X_{n+1}=z+e \mid X_{n}=z\right)=\omega(z, e) .
$$

The quenched law $P_{\omega}^{z}$ is defined to be the law on $\left(\mathbb{Z}^{d}\right)^{\mathbb{N}}$ induced by the kernel $P_{\omega}$ and $P_{\omega}^{z}\left(X_{0}=z\right)=1$. We let $\mathrm{P}^{z}=P \otimes P_{\omega}^{z}$ be the joint law of the environment and the walk, and the annealed law is defined to be its marginal

$$
\mathbb{P}^{z}=\int_{\Omega} P_{\omega}^{z} d P(\omega)
$$

For simplicity, we omit the superscript when the walk starts from zero.

N. Berger: Einstein Institute of Mathematics, The Hebrew University of Jerusalem, Jerusalem, 91904, Israel; e-mail: berger@math.huji.ac.il 
We use the notations $E_{\omega}^{z}$ and $\mathbb{E}^{z}$ for the expectations with respect to the measures $P_{\omega}^{z}$ and $\mathbb{P}^{z}$.

In [11] and [14], Sznitman and Zerner proved that the limiting velocity

$$
\mathbb{v}=\lim _{n \rightarrow \infty} \frac{X_{n}}{n}
$$

exists almost surely. A remaining open problem, which is one of the most important problems in this field, is whether this limiting velocity is always an almost sure constant.

We now introduce three important definitions:

Definition 1. The RWRE is said to be ballistic if the limiting velocity is a non-zero almost sure constant.

Definition 2. The local drift at a point $z$ is defined to be the (quenched) quantity

$$
\Delta_{\omega}(z):=\sum_{e \in\left\{ \pm e_{i}\right\}_{i=1}^{d}} e \omega(z, e)=E_{\omega}^{z}\left(X_{1}-z\right) .
$$

Definition 3. The RWRE is said to be plain nestling if zero is contained in the interior of the convex hull of the support of the random variable $\Delta_{\omega}(0)$. It is said to be marginally nestling if zero is on the boundary of the convex hull of the support, and non-nestling if zero is outside the convex hull of the support.

\subsection{Large deviations for RWRE}

In [12], Varadhan considered large deviations for the sequence of random variables $X_{n} / n$ under the annealed measure $\mathbb{P}$. He showed that a large deviation principle holds with a rate function $F$, and identified the zero set of the function $F$. For the ballistic case with limiting velocity $\mathbb{v}$, Varadhan showed that if the RWRE is non-nestling, then $F^{-1}(0)=$ $\{v\}$, while if the RWRE is plain nestling or marginally nestling, then $F^{-1}(0)=A$, with $A$ being the convex hull of 0 and $\mathbb{v}$. We note here that recently Yilmaz [13] and Peterson [7] obtained more information about the structure of the rate function $F$.

In other words, for every $a \notin A$ and $\epsilon>0$ small enough,

$$
\mathbb{P}\left(\left\|X_{n} / n-a\right\|_{\infty}<\epsilon\right)
$$

decays exponentially with $n$, and for every $a \in A$, (1.2) decays more slowly than exponentially. (Note that the choice of the $\ell^{\infty}$ norm is completely arbitrary, since in our finite-dimensional space, all norms are equivalent.)

It is therefore natural to ask what the decay rate of (1.2) is for $a \in A$.

In the marginally nestling case, Sznitman [8] showed that there exist $C_{1}$ and $C_{2}$ such that

$$
e^{-C_{1} n^{d /(d+2)}}<\mathbb{P}\left(\left\|X_{n} / n-a\right\|_{\infty}<\epsilon\right)<e^{-C_{2} n^{d /(d+2)}}
$$

for large enough $n$. In [8] Sznitman phrased (1.3) in the language of bounds on the distribution of the first regeneration time. However, the way it is presented here follows immediately from Sznitman's result using the appropriate large deviation estimates. 


\subsection{Main goal}

The purpose of this paper is to provide an estimate for the probability in (1.2) in the plain nestling case under some additional assumptions which we specify below.

\subsection{Ballisticity conditions}

In $[9,10]$ Sznitman introduced two criteria for ballisticity of the RWRE, which he called conditions $(T)$ and $\left(T^{\prime}\right)$. In order to define them, we need some preliminary definitions.

Definition 4. Let $\ell \in S^{d-1}$ be a direction in $\mathbb{R}^{d}$. Let $L>0$. For a sequence $\left\{X_{n}\right\}$, we define

$$
T_{L}^{(\ell)}\left(\left\{X_{n}\right\}\right)=\inf \left\{n \geq 0:\left\langle X_{n}, \ell\right\rangle \geq L\right\} .
$$

If no confusion can arise, we may omit $\ell$ and $\left\{X_{n}\right\}$ from this notation.

Equivalently to Definition 4, we also define the first hitting time of a set.

Definition 5. Let $A \subseteq \mathbb{Z}^{d}$. For a sequence $\left\{X_{n}\right\}$, we define

$$
T_{A}\left(\left\{X_{n}\right\}\right)=\inf \left\{n \geq 0: X_{n} \in A\right\} .
$$

Again, we may omit $\left\{X_{n}\right\}$ when no confusion can arise.

We now return to Sznitman's ballisticity conditions. We start by defining the condition $\left(T_{\gamma}\right), 0<\gamma \leq 1$, as follows:

Definition 6. We say that $P$ satisfies condition $\left(T_{\gamma}\right)$ in direction $\ell_{0}$ if for every $\ell$ in a neighborhood of $\ell_{0}$ there exists a constant $C$ such that for every $L$ large enough,

$$
\mathbb{P}\left(T_{L}^{(-\ell)}<T_{L}^{(\ell)}\right)<C \exp \left(-L^{\gamma}\right) .
$$

Definition 7. We say that $P$ satisfies condition $(T)$ if it satisfies condition $\left(T_{1}\right)$. We say that it satisfies condition $\left(T^{\prime}\right)$ if it satisfies condition $\left(T_{\gamma}\right)$ for some $\gamma>1 / 2$.

In [10], it is shown that the conditions $\left(T_{\gamma}\right)_{1 / 2<\gamma<1}$ are all equivalent.

The connection between the conditions mentioned above and ballisticity lies in the following theorem and conjecture:

Theorem 1.1 (Sznitman, [10]). If condition $\left(T^{\prime}\right)$ holds for some $\ell_{0}$, then the RWRE is ballistic, and the limiting velocity $\mathrm{v}$ satisfies $\left\langle\mathrm{v}, \ell_{0}\right\rangle>0$. Furthermore, in this case $\left(T^{\prime}\right)$ holds for all $\ell$ satisfying $\langle\mathrm{v}, \ell\rangle>0$.

Remark. This result was recently improved by Drewitz and Ramírez [4].

Conjecture 1.2 (Sznitman). Condition $\left(T^{\prime}\right)$ is equivalent to ballisticity. 


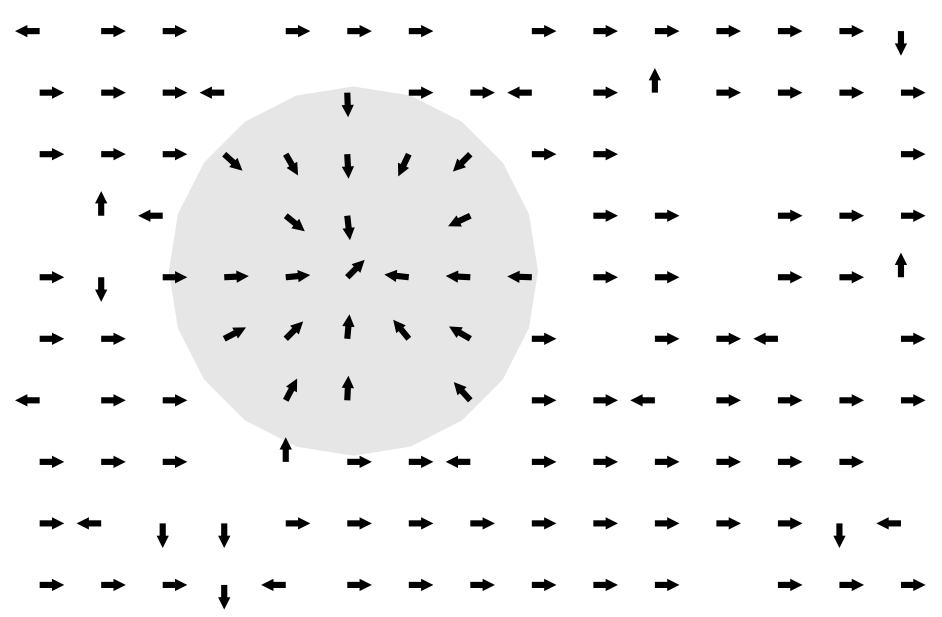

Fig. 1. A naïve trap. In the shaded ball of radius $C \log n$ around the origin all local drifts are pointing towards the origin, while outside the ball the local drift goes mostly to the right. If $C$ is appropriately chosen, then this causes a linear slowdown. The probability that such a configuration exists is exponential in $(\log n)^{d}$.

\subsection{Known slowdown results}

Let $d \geq 2$ and let $a \neq v$ be in the convex hull of 0 and $\mathrm{v}$. Then for $\epsilon>0$ small enough, the following is known.

Theorem 1.3 (Sznitman, [10]). Assume that $P$ is plain nestling, uniformly elliptic and satisfies condition $\left(T^{\prime}\right)$.

(1) There exist $C$ such that for $n$ large enough,

$$
\mathbb{P}\left(\left\|X_{n} / n-a\right\|_{\infty}<\epsilon\right)>e^{-C(\log n)^{d}} .
$$

(2) Let $\alpha<2 d /(d+1)$. There exist $C$ such that for $n$ large enough,

$$
\mathbb{P}\left(\left\|X_{n} / n-a\right\|_{\infty}<\epsilon\right)<e^{-C(\log n)^{\alpha}} .
$$

The easy bound in Theorem 1.3 is (1.5), which follows from the analysis of the so called naïve trap (see Figure 1).

\subsection{Ballisticity under $\left(T_{\gamma}\right)$}

We prove the following result:

Theorem 1.4. Assume that the dimension is at least 4. Fix $\gamma>0$. Under the assumption of uniform ellipticity, if condition $\left(T_{\gamma}\right)$ holds for some $\ell_{0}$, then the RWRE is ballistic, and the limiting velocity $\mathrm{v}$ satisfies $\left\langle\mathrm{v}, \ell_{0}\right\rangle>0$. Furthermore, in this case $\left(T_{\gamma}\right)$ holds for all $\ell$ satisfying $\langle\mathrm{v}, \ell\rangle>0$. 


\subsection{Main results}

Our main result is the following theorem:

Theorem 1.5. Let $d \geq 4$ and $\gamma>0$ and assume that $P$ is uniformly elliptic and satisfies condition $\left(T_{\gamma}\right)$. Let $a \neq v$ be in the convex hull of 0 and $\mathbb{v}$, and let $\epsilon>0$ be small enough so that $\mathbb{v}$ is not in the closed $\epsilon$-neighborhood of a. Let $\alpha<d$. Then for all $n$ large enough,

$$
\mathbb{P}\left(\left\|X_{n} / n-a\right\|_{\infty}<\epsilon\right)<e^{-(\log n)^{\alpha}}
$$

Comparing Theorem 1.5 and (1.5) shows that the remaining gap between the upper and the lower bounds is quite small.

Theorem 1.5 deals with the annealed probability of slowdown. However, one can deduce from it a quenched bound.

Corollary 1.6. With the same assumptions as in Theorem 1.5, for every $\alpha<d$, almost every $\omega$ and every large enough $n$,

$$
P_{\omega}\left(\left\|X_{n} / n-a\right\|_{\infty}<\epsilon\right)<\exp \left(-\frac{n}{\exp \left((\log n)^{\alpha^{-1}}\right)}\right) .
$$

Again, compare (1.8) to the known lower bound

$$
P_{\omega}\left(\left\|X_{n} / n-a\right\|_{\infty}<\epsilon\right)>\exp \left(-\frac{C n}{\exp \left((\log n)^{d^{-1}}\right)}\right),
$$

which is proven in [8]. Corollary 1.6 follows from Theorem 1.5 using the method developed by Gantert and Zeitouni [6] to transfer annealed slowdown estimates into quenched ones. This method was adjusted to the multi-dimensional case by Sznitman [8]. The proof of Corollary 1.6 is identical to the proof of (5.45) in [8], and is omitted.

\subsection{Remark about lower dimensions}

In this paper we only prove Theorem 1.5 for dimensions 4 and higher. Here we discuss the situation in lower dimensions.

For $d=1$, the annealed slowdown probability was calculated by Dembo, Peres and Zeitouni [3] in 1996 and the quenched slowdown probability was calculated by Gantert and Zeitouni [6] in 1998. These results give bounds that are significantly sharper than the bounds in Theorem 1.5 and Corollary 1.6. Nevertheless, a comparison between the results shows that the estimates in the present paper are true for dimension 1.

I conjecture that the results in this paper hold for dimensions 2 and 3. The difficulty in the proof occurs in Proposition 4.5, which is currently only proved for dimensions 4 and higher. In dimension 3 I expect that a more sophisticated version of the arguments in this paper should be able to work. In dimension 2, Proposition 4.5(2) does not hold, and therefore a new idea is needed. 


\subsection{Structure of the paper}

Section 2 brings the definition of regeneration times as introduced in [11]. We then reformulate Theorem 1.5 in the language of regenerations and get Proposition 2.2. Then in Section 3 we introduce some very useful notation and give some basic definitions. In Section 4 we give a number of CLT type results. In particular, we give an almost local version of the quenched central limit theorem (Proposition 4.5) and a general lemma about sums of approximately Gaussian variables (Lemma 4.16). In Section 5 we reformulate Theorem 1.5 as a statement about quenched exit properties from a large box. The first half of this construction is very similar to Sznitman's construction in [10]. Then in Section 6 we define an auxiliary walk $\left\{Y_{n}\right\}$ and explore its connection with the original walk $\left\{X_{n}\right\}$. In Section 7 we define an event regarding the walk $\left\{Y_{n}\right\}$, and in Section 8 we use all that information in order to prove the main result.

\subsection{Remark about the writing style}

In order to avoid notational overload, language is abused in three ways in this paper: (a) the value of a constant $C$ may change from one line to the next, (b) some of the inequalities only hold for $n$ large enough, without explicit mention, and (c) for a probability measure $\mu$ on $\mathbb{Z}^{d}$, we use the symbol $E_{\mu}$ for the expectation $\sum_{x} x \mu(x)$.

In addition we use the highly convenient notations from computer science $O(n), o(n)$, $\Omega(n)$ and $\xi(n)$, whose meanings are as described in the table below. Note that computer scientists write $\omega$ rather than $\xi$. However, due to the use of the letter $\omega$ for the environment in this paper, we use $\xi$ as described below.

\begin{tabular}{ll}
\hline Symbol & Meaning \\
\hline$k=O(n)$ & as the parameter goes to infinity, $\lim \sup \frac{k}{n}<\infty$ \\
$k=o(n)$ & as the parameter goes to infinity, $\lim \frac{k}{n}=0$ \\
$k=\Omega(n)$ & as the parameter goes to infinity, $\lim \inf \frac{k}{n}>0$ \\
$k=\xi(n)$ & as the parameter goes to infinity, $\lim \frac{k}{n}=\infty$ \\
\hline
\end{tabular}

For example, if we write $f(N)=N^{-\xi(1)}$, we mean that as $N$ goes to infinity, $f(N)$ goes to zero faster than any power of $N$.

Whenever the norm sign $\|\cdot\|$ appears without mentioning which norm we are referring to, we refer to the $\ell^{\infty}$ norm on $\mathbb{Z}^{d}$.

\section{Regeneration times}

We first define the notion of a regeneration time. Our definition is slightly different from that given by Sznitman and Zerner [11]. Nevertheless, all the lemmas that we quote from [10] and [11] and collect in Theorem 2.1 apply equally well to the definition below. 
Definition 8. Let $\left\{X_{n}\right\}$ be a nearest-neighbor sequence in $\mathbb{Z}^{d}$, and let $\ell \in S^{d-1}$ be a direction. We say that $t$ is a regeneration time for $\left\{X_{n}\right\}$ in direction $\ell$ if the following hold:

(1) $\left\langle X_{s}, \ell\right\rangle<\left\langle X_{t}, \ell\right\rangle$ for every $s<t$.

(2) $\left\langle X_{t+1}, \ell\right\rangle>\left\langle X_{t}, \ell\right\rangle$.

(3) $\left\langle X_{s}, \ell\right\rangle>\left\langle X_{t+1}, \ell\right\rangle$ for every $s>t+1$.

Theorem 2.1 ([11, 10]). Assume that $P$ satisfies condition $\left(T_{\gamma}\right)$ in direction $\ell_{0}$ for some $\gamma>0$. Then:

(1) With probability 1, there exist infinitely many regeneration times. We call them $\tau_{1}<$ $\tau_{2}<\cdots$.

(2) The ensemble

$$
\left\{\left(\tau_{n+1}-\tau_{n}, X_{\tau_{n+1}}-X_{\tau_{n}}\right)\right\}_{n \geq 1}
$$

is an i.i.d. ensemble under the annealed measure.

(3) There exists $C$ such that for every n,

$$
\mathbb{P}\left(\tau_{2}-\tau_{1}=n\right) \leq C \mathbb{P}\left(\tau_{1}=n\right),
$$

and for every $y \in \mathbb{Z}^{d}$,

$$
\mathbb{P}\left(X_{\tau_{2}}-X_{\tau_{1}}=y\right) \leq C \mathbb{P}\left(X_{\tau_{1}}=y\right) .
$$

(4) There exists $C$ such that for every $n$,

$$
\mathbb{P}\left(\exists_{k \leq \tau_{1}}\left\|X_{k}\right\|>n\right) \leq e^{-C n^{\gamma}} .
$$

The main technical statement in this paper is the following proposition.

Proposition 2.2. For any $\gamma>0$, if the dimension $d$ is greater than or equal to 4 , and $\mathbb{P}$ satisfies condition $\left(T_{\gamma}\right)$ in one of the $2 d$ principle directions, then for every $\alpha<d$ and every u large enough,

$$
\mathbb{P}\left(\tau_{1}>u\right) \leq \exp \left(-(\log u)^{\alpha}\right) .
$$

We now show how to prove Theorem 1.5 assuming Proposition 2.2. The rest of the paper will be dedicated to the proof of Proposition 2.2.

Proof of Theorem 1.4 assuming Proposition 2.2. Theorem 1.4 follows from Proposition 2.2 exactly the same way ballisticity is proved in [11] and [10].

Proof of Theorem 1.5 assuming Proposition 2.2. Fix $\alpha<d$. Assume without loss of generality that $\left\langle\mathrm{v}, e_{1}\right\rangle>0$. Note that in this case condition $\left(T_{\gamma}\right)$ holds with respect to the direction $e_{1}$. For simplicity, in this proof we denote $\bar{x}=\left\langle x, e_{1}\right\rangle$ for every $x \in \mathbb{R}^{d}$. Fix $a$ and $\epsilon$ as in the statement of Theorem 1.5. Let $\rho=\mathbb{E}\left(\tau_{2}-\tau_{1}\right)$ and let $\beta=\mathbb{E}\left(\bar{X}_{\tau_{2}}-\bar{X}_{\tau_{1}}\right)$. Let $r$ be such that $r<\overline{\mathrm{v}}$ but $r>\bar{x}$ for every $x$ in the $\epsilon$-neighborhood of $a$. Then it is sufficient to show that for all $n$ large enough,

$$
\mathbb{P}\left(\bar{X}_{n}<r n\right)<e^{-(\log n)^{\alpha}} .
$$


Choose $b$ so that $r / \overline{\mathrm{V}}<b<1$, and let $m=n b / \rho$. Then

$$
\mathbb{P}\left(\bar{X}_{n}<r n\right) \leq \mathbb{P}\left(\tau_{m+1}>n\right)+\mathbb{P}\left(\bar{X}_{\tau_{m+1}}<r n\right) .
$$

Now, remembering that $\overline{\mathrm{v}}=\beta / \rho$,

$$
\mathbb{P}\left(\bar{X}_{\tau_{m+1}}<r n\right) \leq \mathbb{P}\left(\bar{X}_{\tau_{m+1}}-\bar{X}_{\tau_{1}}<r n\right)=\mathbb{P}\left(\sum_{k=1}^{m} \bar{X}_{\tau_{k+1}}-\bar{X}_{\tau_{k}}<\frac{r}{b} \rho m\right) .
$$

Remembering that the sequence $\left\{\bar{X}_{\tau_{k+1}}-\bar{X}_{\tau_{k}}\right\}$ is i.i.d. and that $\bar{X}_{\tau_{k+1}}-\bar{X}_{\tau_{k}}$ is positive and its expectation $\beta$ is larger than $(r / b) \rho$, we get

$$
\mathbb{P}\left(\bar{X}_{\tau_{m+1}}<r n\right)<e^{-C n}
$$

for some constant $C$.

We now estimate $\mathbb{P}\left(\tau_{m+1}>n\right)$. Let $A$ be the event that $\tau_{k+1}-\tau_{k}<n^{1 / 8}$ for all $k=1, \ldots, m$ and that $\tau_{1}<n^{1 / 8}$. Then

$$
\mathbb{P}\left(\tau_{m+1}>n\right) \leq \mathbb{P}\left(A^{c}\right)+\mathbb{P}\left(\tau_{m+1}>n \mid A\right) .
$$

Fix $\alpha^{\prime}$ between $\alpha$ and $d$. Then, by Theorem 2.1(3) and Proposition 2.2, for all $n$ large enough,

$$
\mathbb{P}\left(A^{c}\right) \leq e^{-\left(\log n^{1 / 8}\right)^{\alpha^{\prime}}}+m C e^{-\left(\log n^{1 / 8}\right)^{\alpha^{\prime}}} \leq \frac{1}{2} e^{-(\log n)^{\alpha}} .
$$

Conditioned on $A$, the variables $\tau_{k+1}-\tau_{k}$ are independent, bounded by $n^{1 / 8}$ and their expectation is less than $\rho$. Then by Azuma's inequality, for $n$ large enough,

$$
\begin{aligned}
\mathbb{P}\left(\tau_{m+1}>n \mid A\right) & \leq \mathbb{P}\left(\tau_{m+1}-\tau_{1}>n-n^{1 / 8} \mid A\right) \leq \exp \left(-\frac{\left(n-n^{1 / 8}-\rho m\right)^{2}}{2 m n^{1 / 4}}\right) \\
& \leq \exp \left(-n^{3 / 4}\right) .
\end{aligned}
$$

(2.2) follows.

\section{Preliminaries}

We define $R_{k}(N)=\left[\exp \left((\log \log N)^{k+1}\right)\right]$ and $R(N)=R_{1}(N)$. Note that $R_{0}(N)=$ $[\log N]$ and that for all $k, M$ and all large enough $N$,

$$
R_{k}^{M}(N)<R_{k+1}(N)<N .
$$

We let

$$
\vartheta:=\lim _{n \rightarrow \infty} X_{n} /\left\|X_{n}\right\|
$$

be the direction of the speed. Note that the existence of $\vartheta$ follows from $T_{\gamma}$ even without the ballisticity assumption, and is always non-zero. We assume without loss of generality that $\left\langle\vartheta, e_{1}\right\rangle>0$. Note that, by the results of [9] and [10], $\left(T_{\gamma}\right)$ holds both in direction $e_{1}$ and in direction $\vartheta$. 
Definition 9. For $z \in \mathbb{Z}^{d}$ and $N \in \mathbb{N}$, we define the basic block of size $N$ around $z$ to be

$$
\mathcal{P}(z, N):=\left\{x \in \mathbb{Z}^{d}:\left|\left\langle x, e_{1}\right\rangle-\left\langle z, e_{1}\right\rangle\right|<N^{2},\|x-u(z, x)\|_{\infty}<N R_{5}(N)\right\}
$$

where

$$
u(z, x):=z+\vartheta \cdot \frac{\left\langle x-z, e_{1}\right\rangle}{\left\langle\vartheta, e_{1}\right\rangle} .
$$

The middle third of $\mathcal{P}(z, N)$ is defined to be

$$
\tilde{\mathcal{P}}(z, N):=\left\{x \in \mathbb{Z}^{d}:\left|\left\langle x, e_{1}\right\rangle-\left\langle z, e_{1}\right\rangle\right|<\frac{1}{3} N^{2},\|x-u(z, x)\|_{\infty}<\frac{1}{3} N R_{5}(N)\right\}
$$

We let

$$
\begin{aligned}
\partial \mathcal{P}(z, N) & :=\left\{x \in \mathbb{Z}^{d} \backslash \mathcal{P}(z, N): \exists_{y \in \mathcal{P}(z, N)}\|x-y\|_{1}=1\right\}, \\
\partial^{+} \mathcal{P}(z, N) & :=\left\{x \in \partial \mathcal{P}(z, N):\left\langle x, e_{1}\right\rangle-\left\langle z, e_{1}\right\rangle=N^{2}\right\} .
\end{aligned}
$$

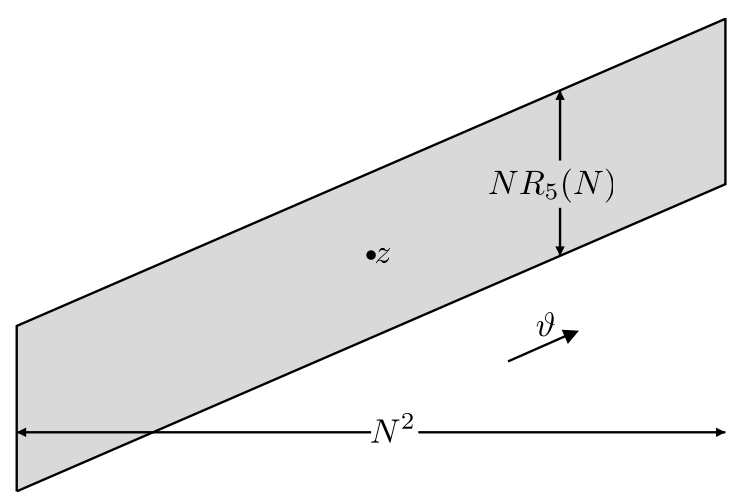

Fig. 2. The basic block $\mathcal{P}(z, N)$.

Definition 10. The basic lattice of size $N$ is defined to be

$$
\mathcal{L}_{N}:=N^{2} \mathbb{Z} \times\left(\left[\frac{N R_{5}(N)}{4}\right] \mathbb{Z}\right)^{d-1}
$$

The following simple fact will be useful in what follows.

Lemma 3.1. (1) For every $x \in N^{2} \mathbb{Z} \times \mathbb{Z}^{d-1}$ there exists $z \in \mathcal{L}_{N}$ such that $x \in \tilde{\mathcal{P}}(z, N)$. (2) $\mathcal{L}_{N}$ can be represented as the disjoint union of $9^{d}$ lattices such that if $\mathcal{L}$ is one of these lattices then for every $z_{1} \neq z_{2}$ in $\mathcal{L}$,

$$
\mathcal{P}\left(z_{1}, N\right) \cap \mathcal{P}\left(z_{2}, N\right)=\emptyset .
$$

\section{CLT type estimates}

In this section we derive two CLT type estimates that will be important for the proof of the main result. Throughout this section, we assume that our RWRE model satisfies the following requirements: 
1. There exists $\gamma>0$ such that $T_{\gamma}$ holds.

2. The RWRE is uniformly elliptic.

3. The dimension $d$ is at least 4 .

We begin with some preliminary estimates in Subsections 4.1 and 4.2 , and then prove CLT type estimates in Subsections 4.3 and 4.4.

\subsection{Regeneration radii}

We define $X^{*(1)}:=\max \left\{\left\|X_{t}-X_{0}\right\|: 0 \leq t \leq \tau_{1}\right\}$, and for $n>1$ we let $X^{*(n)}:=$ $\max \left\{\left\|X_{t}-X_{\tau_{n-1}}\right\|: \tau_{n-1} \leq t \leq \tau_{n}\right\}$. The following lemma appears in [10].

Lemma 4.1. Let $\gamma>0$ and assume that condition $\left(T_{\gamma}\right)$ holds. Then there exist $C$ and $c$ such that for every $L$ and $n=1,2, \ldots$,

$$
\mathbb{P}\left(X^{*(n)}>L\right) \leq C e^{-c L^{\gamma}}
$$

for every $n$.

We call $X^{*(n)}$ the radius of the $n$-th regeneration. Recall that $R(N)=R_{1}(N)=$ $\left[e^{(\log \log N)^{2}}\right]=\left[(\log N)^{\log \log N}\right]$. Let $A_{N}\left(\left\{X_{n}\right\}\right)$ be the event that the radii of the first $N$ regenerations are all smaller than $R(N)$, namely

$$
A_{N}\left(\left\{X_{n}\right\}\right):=\left\{\forall_{1 \leq n \leq N}, X^{*(n)}<R(N)\right\} .
$$

Then, by Lemma 4.1,

$$
\mathbb{P}\left(A_{N}\left(\left\{X_{n}\right\}\right)\right) \geq 1-C N e^{-c R(N)^{\gamma}}=1-N^{-\xi(1)} .
$$

\subsection{Derivatives of the annealed exit distribution}

In this subsection we show the following result on the annealed exit distribution from a block. The proof is standard and straightforward using regenerations.

Lemma 4.2. Assume the assumptions $1-3$ above hold. Fix $z \in \mathbb{Z}^{d}$ and $N$, and let $z_{1} \in$ $\tilde{\mathcal{P}}(z, N)$. Let $\left\{X_{n}\right\}$ be an RWRE starting at $z_{1}$, and let $u:=X_{T_{\partial \mathcal{P}(z, N)}}$. Then, for large enough $N$ :

(1) $\mathbb{P}^{z_{1}}\left(u \notin \partial^{+} \mathcal{P}(z, N)\right) \leq e^{-R_{5}(N)}+\mathbb{P}\left(A_{N}^{c}\right)=N^{-\xi(1)}$, where $A_{N}$ is as defined in (4.2).

(2) For every $x$ in $\partial^{+} \mathcal{P}(z, N)$,

$$
\mathbb{P}^{z_{1}}(u=x)<C N^{1-d} .
$$

(3) For every $x$ and $y$ in $\partial^{+} \mathcal{P}(z, N)$ such that $\|x-y\|_{1}=1$,

$$
\left|\mathbb{P}^{z_{1}}(u=x)-\mathbb{P}^{z_{1}}(u=y)\right|<C N^{-d} .
$$

(4) Let $\left\{X_{n}^{\prime}\right\}$ be an RWRE starting at $z_{1}+e_{1}$, and let $u^{\prime}:=X_{T_{\partial \mathcal{P}(z, N)}^{\prime}}$. Then for every $x$ in $\partial^{+} \mathcal{P}(z, N)$,

$$
\left|\mathbb{P}^{z_{1}}(u=x)-\mathbb{P}^{z_{1}+e_{1}}\left(u^{\prime}=x\right)\right|<C N^{-d} .
$$


(5) For every $x, y$ and $w$ in $\partial^{+} \mathcal{P}(z, N)$ such that $\|x-y\|_{1}=1$ and $w-y=y-x$,

$$
\left|\mathbb{P}^{z_{1}}(u=x)+\mathbb{P}^{z_{1}}(u=w)-2 \mathbb{P}^{z_{1}}(u=y)\right|<C N^{-d-1} .
$$

(6) For every $x, y$ and $w$ and $o$ in $\partial^{+} \mathcal{P}(z, N)$ such that there exist $i \neq j$ with $x-y=$ $w-v=e_{i}$ and $x-w=y-o=e_{j}$,

$$
\left|\mathbb{P}^{z_{1}}(u=x)+\mathbb{P}^{z_{1}}(u=o)-\mathbb{P}^{z_{1}}(u=y)-\mathbb{P}^{z_{1}}(u=w)\right|<C N^{-d-1} .
$$

Proof. To prove (1), we need to show that

$$
\mathbb{P}^{z_{1}}\left(u \notin \partial^{+} \mathcal{P}(z, N) \mid A_{N}\right) \leq e^{-R_{5}(N)} .
$$

To this end, note that conditioned on $A_{N}$, the regenerations are independent and are all bounded by $R(N)$. For $k=1, \ldots, N$ we now estimate the difference between $\mathbb{E}^{z_{1}}\left(X_{\tau_{k}} \mid A_{N}\right)$ and $\mathbb{E}^{z_{1}}\left(X_{\tau_{k}}\right)$. Let $\Xi_{k}=X_{\tau_{k}}-X_{\tau_{k-1}}$ with $\Xi_{1}=X_{\tau_{1}}$. Remember that $\mathbb{P}^{z 1}\left(A_{N}^{c}\right)=N^{-\xi(1)}$. For a given $k$,

$$
\begin{aligned}
\mathbb{E}^{z_{1}}\left(\left\|\Xi_{k}\right\| \cdot \mathbf{1}_{A_{N}^{c}}\right) & \leq \mathbb{E}^{z_{1}}\left(\left\|\Xi_{k}\right\| \cdot \mathbf{1}_{\exists_{j \neq k} X^{\star(j)} \geq R(N)}\right)+\mathbb{E}^{z_{1}}\left(\left\|\Xi_{k}\right\| \cdot \mathbf{1}_{X^{\star(k)} \geq R(N)}\right) \\
& \leq \mathbb{P}^{z_{1}}\left(A_{N}^{c}\right) \mathbb{E}^{z_{1}}\left(\left\|\Xi_{k}\right\|\right)+\sum_{h>R(N)} h \mathbb{P}^{z_{1}}\left(X^{\star(k)}=h\right) \\
& \leq \mathbb{P}^{z_{1}}\left(A_{N}^{c}\right) \mathbb{E}^{z_{1}}\left(\left\|\Xi_{k}\right\|\right)+\sum_{h>R(N)} h e^{-c h^{\gamma}}=N^{-\xi(1)}
\end{aligned}
$$

Therefore, for every $k=1, \ldots, N$,

$$
\begin{aligned}
\| \mathbb{E}^{z_{1}}\left(\Xi_{k} \mid A_{N}\right) & -\mathbb{E}^{z_{1}}\left(\Xi_{k}\right) \| \\
& \leq\left\|\mathbb{E}^{z_{1}}\left(\Xi_{k} \cdot \mathbf{1}_{A_{N}}\right)-\mathbb{E}^{z_{1}}\left(\Xi_{k}\right)\right\|+\left\|\mathbb{E}^{z_{1}}\left(\Xi_{k} \mid A_{N}\right)-\mathbb{E}^{z_{1}}\left(\Xi_{k} \cdot \mathbf{1}_{A_{N}}\right)\right\| \\
& \leq \mathbb{E}^{z_{1}}\left(\left\|\Xi_{k}\right\| \cdot \mathbf{1}_{A_{N}^{c}}\right)+\mathbb{E}^{z_{1}}\left(\left\|\Xi_{k}\right\| \mid A_{N}\right) \mathbb{P}^{z_{1}}\left(A_{N}^{c}\right)=N^{-\xi(1)} .
\end{aligned}
$$

Hence $\left\|\mathbb{E}^{z_{1}}\left(X_{\tau_{k}} \mid A_{N}\right)-\mathbb{E}^{z_{1}}\left(X_{\tau_{k}}\right)\right\|=N^{-\xi(1)}$, again for every $k=1, \ldots, N$.

Now, using Azuma's inequality,

$$
\begin{aligned}
\mathbb{P}^{z_{1}}\left(u \notin \partial^{+} \mathcal{P}(z, N) \mid A_{N}\right) & \leq \sum_{k=1}^{N^{2}} \mathbb{P}^{z_{1}}\left[\left\|X_{\tau_{k}}-\mathbb{E}^{z_{1}}\left(X_{\tau_{k}} \mid A_{N}\right)\right\|_{\infty}>\frac{1}{4} N R_{5}(N)\right] \\
& \leq d \sum_{k=1}^{N^{2}} \exp \left(\frac{-N^{2} R_{5}^{2}(N) / 16}{2 k R^{2}(N)}\right) \leq e^{-R_{5}(N)}
\end{aligned}
$$

for $N$ large enough.

To prove (2)-(6) we need the following standard claim.

Claim 4.3. Let $\left\{Y_{i}\right\}_{i=1}^{\infty}$ be d-dimensional independent random variables, with joint distribution $\mathbf{P}$, such that $\left\{Y_{n}\right\}_{n \geq 2}$ are identically distributed and such that there exists $v \in \mathbb{Z}^{d}$ such that $\mathbf{P}\left(Y_{2}=v\right)>0$ and $\mathbf{P}\left(Y_{2}=v+e_{i}\right)>0$ for $i=1, \ldots, d$. Let $S_{n}=\sum_{i=1}^{n} Y_{i}$. Then there exists $C<\infty$ which is determined by the distributions of $Y_{1}$ and $Y_{2}$ such that for every $n$ and every $x, y$ and $w$ with $\|x-y\|_{1}=1$ and $w-y=y-x$,

$$
\mathbf{P}\left(S_{n}=x\right) \leq C n^{-d / 2} \text {, }
$$




$$
\begin{gathered}
\left|\mathbf{P}\left(S_{n}=x\right)-\mathbf{P}\left(S_{n}=y\right)\right| \leq C n^{(-1-d) / 2}, \\
\left|\mathbf{P}\left(S_{n}=x\right)+\mathbf{P}\left(S_{n}=w\right)-2 \mathbf{P}\left(S_{n}=y\right)\right| \leq C n^{(-2-d) / 2} .
\end{gathered}
$$

In addition, for every $x, y$ and $w$ and $o$ such that there exist $i \neq j$ with $x-y=w-v=e_{i}$ and $x-w=y-o=e_{j}$,

$$
\left|\mathbf{P}\left(S_{n}=x\right)+\mathbf{P}\left(S_{n}=o\right)-\mathbf{P}\left(S_{n}=y\right)-\mathbf{P}\left(S_{n}=w\right)\right|<C n^{(-2-d) / 2} .
$$

We now use Claim 4.3; we will prove it later. For $k$ and $l$ in $\mathbb{N}$, we let $B(l, k)$ be the event that $\left\langle X_{\tau_{k}}, e_{1}\right\rangle=l$, we let $B(l)=\bigcup_{k} B(l, k)$ and we let $\hat{B}(l)$ be the event

$$
\hat{B}(l):=B(l) \cap \bigcap_{j=l+1}^{N^{2}} B^{c}(j) .
$$

In addition, we define $Z_{l}=X_{T_{l}}$. Then, for $x$ and $y$ such that $\|x-y\|_{1}=1$ and $\left\langle x, e_{1}\right\rangle=$ $\left\langle y, e_{1}\right\rangle=l$,

$$
\begin{aligned}
\mathbb{P}^{z_{1}}\left(Z_{l}=x \mid \hat{B}(l)\right)-\mathbb{P}^{z_{1}}\left(Z_{l}=y \mid \hat{B}(l)\right) & =\mathbb{P}^{z_{1}}\left(Z_{l}=x \mid B(l)\right)-\mathbb{P}^{z_{1}}\left(Z_{l}=y \mid B(l)\right) \\
& =\frac{1}{\mathbb{P}^{z_{1}}(B(l))} \sum_{k}\left(\mathbb{P}^{z_{1}}\left(X_{\tau_{k}}=x\right)-\mathbb{P}^{z_{1}}\left(X_{\tau_{k}}=y\right)\right) .
\end{aligned}
$$

Let

$$
M=\frac{l}{\mathbb{E}^{z_{1}}\left[\left\langle X_{\tau_{2}}-X_{\tau_{1}}, e_{1}\right\rangle\right]} .
$$

For $x$ and $y$ satisfying $\left\langle x, e_{1}\right\rangle=\left\langle y, e_{1}\right\rangle=l$ and $\|x-y\|=1$, and for $k \in \mathbb{N}$, we now estimate

$$
\left|\mathbb{P}^{z_{1}}\left(X_{\tau_{k}}=x\right)-\mathbb{P}^{z_{1}}\left(X_{\tau_{k}}=y\right)\right| .
$$

We consider two different cases: $k \geq M$ and $k<M$. Assume first that $k \geq M$. Then either $\left\langle X_{\tau_{[k / 2]}}, e_{1}\right\rangle \leq l / 2$ or $\left\langle X_{\tau_{k}}-X_{\tau_{[k / 2]}}, e_{1}\right\rangle \leq l / 2$. So

$$
\begin{aligned}
& \left|\mathbb{P}^{z_{1}}\left(X_{\tau_{k}}=x\right)-\mathbb{P}^{z_{1}}\left(X_{\tau_{k}}=y\right)\right| \\
& \leq \mathbb{P}^{z_{1}}\left(X_{\tau_{k}}=x ;\left\langle X_{\tau_{[k / 2]}}, e_{1}\right\rangle \leq l / 2\right)-\mathbb{P}^{z_{1}}\left(X_{\tau_{k}}=y ;\left\langle X_{\tau_{[k / 2]}}, e_{1}\right\rangle \leq l / 2\right) \\
& \quad+\mathbb{P}^{z_{1}}\left(X_{\tau_{k}}=x ;\left\langle X_{\tau_{k}}-X_{\tau_{[k / 2]}}, e_{1}\right\rangle \leq l / 2\right) \\
& \quad-\mathbb{P}^{z_{1}}\left(X_{\tau_{k}}=y ;\left\langle X_{\tau_{k}}-X_{\tau_{[k / 2]}}, e_{1}\right\rangle \leq l / 2\right) .
\end{aligned}
$$

We now estimate (4.8) ((4.9) is estimated the same way):

$$
\begin{aligned}
& \mathbb{P}^{z_{1}}\left(X_{\tau_{k}}=x ;\left\langle X_{\tau_{[k / 2]}}, e_{1}\right\rangle \leq l / 2\right)-\mathbb{P}^{z_{1}}\left(X_{\tau_{k}}=y ;\left\langle X_{\tau_{[k / 2]}}, e_{1}\right\rangle \leq l / 2\right) \\
& =\sum_{w:\left\langle w, e_{1}\right\rangle \leq l / 2} \mathbb{P}^{z_{1}}\left(X_{\tau_{[k / 2]}}=w\right)\left[\mathbb{P}^{z_{1}}\left(X_{\tau_{k}}=x \mid X_{\tau_{[k / 2]}}=w\right)-\mathbb{P}^{z_{1}}\left(X_{\tau_{k}}=y \mid X_{\tau_{[k / 2]}}=w\right)\right] \\
& \leq C k^{(-1-d) / 2} \sum_{w:\left\langle w, e_{1}\right\rangle \leq l / 2} \mathbb{P}^{z_{1}}\left(X_{\tau_{[k / 2]}}=w\right) \\
& =C k^{(-1-d) / 2} \mathbb{P}^{z_{1}}\left(\left\langle X_{\tau_{[k / 2]}}, e_{1}\right\rangle \leq l / 2\right)
\end{aligned}
$$

where the inequality follows from (4.5). 
With a similar calculation for $k<M$, we get

$$
\begin{aligned}
& \left|\mathbb{P}^{z_{1}}\left(Z_{l}=x \mid \hat{B}(l)\right)-\mathbb{P}^{z_{1}}\left(Z_{l}=y \mid \hat{B}(l)\right)\right| \\
& \leq C \sum_{k=1}^{M} k^{(-1-d) / 2} \mathbb{P}^{z_{1}}\left(\left\langle X_{\tau_{[k / 2]}}, e_{1}\right\rangle \geq l / 2\right)+C \sum_{k=M}^{\infty} k^{(-1-d) / 2} \mathbb{P}^{z_{1}}\left(\left\langle X_{\tau_{[k / 2]}}, e_{1}\right\rangle \leq l / 2\right) .
\end{aligned}
$$

From Lemma 4.1 we learn that $X^{\star(n)}$ has (in particular) a finite $2 d$ moment, and from standard estimates on sums of i.i.d. variables (namely that the $2 d$ moment of the sum of $k$ i.i.d. mean zero variables grows like $O\left(k^{d}\right)$ ), we find that for $k<M$,

$$
\mathbb{P}^{z_{1}}\left(\left\langle X_{\tau_{[k / 2]}}, e_{1}\right\rangle \geq l / 2\right) \leq \min \left[1, C \frac{k^{d}}{(M-k)^{2 d}}\right],
$$

and for $k \geq M$,

$$
\mathbb{P}^{z_{1}}\left(\left\langle X_{\tau_{[k / 2]}}, e_{1}\right\rangle \leq l / 2\right) \leq \min \left[1, C \frac{k^{d}}{(k-M)^{2 d}}\right]=\min \left[1, C \frac{k^{d}}{(M-k)^{2 d}}\right] .
$$

Combining this with (4.11) we get

$$
\begin{aligned}
&\left|\mathbb{P}^{z 1}\left(Z_{l}=x \mid \hat{B}(l)\right)-\mathbb{P}^{z_{1}}\left(Z_{l}=y \mid \hat{B}(l)\right)\right| \\
& \leq C \sum_{k=1}^{M} k^{(-1-d) / 2} \min \left[1, C \frac{k^{d}}{(M-k)^{2 d}}\right] \\
&+C \sum_{k=M}^{\infty} k^{(-1-d) / 2} \min \left[1, C \frac{k^{d}}{(k-M)^{2 d}}\right] \\
& \leq C \sum_{k=1}^{[M / 2]} k^{(-1-d) / 2} \min \left[1, C \frac{k^{d}}{(M-k)^{2 d}}\right] \\
&+C \sum_{k=[M / 2]+1}^{M-\left[M^{1 / 2}\right]} k^{(-1-d) / 2} \min \left[1, C \frac{k^{d}}{(M-k)^{2 d}}\right] \\
&+C \sum_{k=M-\left[M^{1 / 2}\right]+1}^{M+\left[M^{1 / 2}\right]} k^{(-1-d) / 2} \min \left[1, C \frac{k^{d}}{(M-k)^{2 d}}\right] \\
&+C \sum_{k=M+\left[M^{1 / 2}\right]+1}^{\infty} k^{(-1-d) / 2} \min \left[1, C \frac{k^{d}}{(k-M)^{2 d}}\right] \\
& \leq C l^{-d / 2} .
\end{aligned}
$$

To see the inequality (4.16), we bound each of the four sums (4.12)-(4.15) by $\mathrm{Cl}^{-d / 2}$. For the expression in (4.12), we note that we have $O(l)$ summands, each of which is 
bounded by $O\left(l^{-d}\right)$, so the sum is bounded by $O\left(l^{1-d}\right)$. The expression in (4.13) is (up to a constant) bounded by

$$
\begin{aligned}
\int_{M / 2}^{M-\sqrt{M}} x^{(d-1) / 2}(M-x)^{-2 d} d x & \leq M^{(d-1) / 2} \int_{\sqrt{M}}^{M / 2} y^{-2 d} d y \leq C M^{(d-1) / 2} \sqrt{M}^{1-2 d} \\
& =O\left(l^{-d / 2}\right) .
\end{aligned}
$$

The expression in (4.14) contains $O(\sqrt{l})$ summands, each of which is $O\left(l^{(-1-d) / 2}\right)$, so the sum is $O\left(l^{-d / 2}\right)$. The expression in (4.15) is taken care of similarly to the one in (4.13) - it is bounded by a constant times the integral

$$
\begin{aligned}
\int_{M+\sqrt{M}}^{\infty} x^{(d-1) / 2}(x-M)^{-2 d} d x & \\
& =\int_{M+\sqrt{M}}^{2 M} x^{(d-1) / 2}(x-M)^{-2 d} d x+\int_{2 M}^{\infty} x^{(d-1) / 2}(x-M)^{-2 d} d x \\
\leq & C M^{(d-1) / 2} \int_{\sqrt{M}}^{M} y^{-2 d} d y+C \int_{M}^{\infty} y^{(d-1) / 2-2 d} d y=O\left(l^{-d / 2}\right),
\end{aligned}
$$

where we substituted, for both integrals, $y=x-M$.

Let $H_{l}=\left\{w:\left\langle w, e_{1}\right\rangle=l\right\}$. Now,

$$
\mathbb{P}^{z_{1}}(u=x)=\sum_{l \leq N^{2}} \mathbb{P}^{z_{1}}(\hat{B}(l)) \sum_{w \in H_{l}} \mathbb{P}^{z_{1}}\left(X_{T_{l}}=w \mid \hat{B}(l)\right) \mathbb{P}^{z_{1}}\left(u=x \mid \hat{B}(l) ; X_{T_{l}}=w\right)
$$

and, using shift invariance and the fact that we condition on the occurrence of a regeneration at $l$, for $y=x+e_{j}(j \neq 1)$,

$\mathbb{P}^{z_{1}}(u=y)=\sum_{l \leq N^{2}} \mathbb{P}^{z_{1}}(\hat{B}(l)) \sum_{w \in H_{l}} \mathbb{P}^{z_{1}}\left(X_{T_{l}}=w+e_{j} \mid \hat{B}(l)\right) \mathbb{P}^{z_{1}}\left(u=x \mid \hat{B}(l) ; X_{T_{l}}=w\right)$.

Noting that due to shift invariance,

$$
\sum_{w \in H_{l}} \mathbb{P}^{z_{1}}\left(u=x \mid \hat{B}(l) ; X_{T_{l}}=w\right)=1,
$$

we get

$$
\begin{aligned}
\mid \mathbb{P}^{z_{1}}(u= & x)-\mathbb{P}^{z_{1}}(u=y) \mid \\
& \leq \sum_{l \leq N^{2}} \mathbb{P}^{z_{1}}(\hat{B}(l)) \max _{w \in H_{l}}\left|\mathbb{P}^{z_{1}}\left(X_{T_{l}}=w+e_{j} \mid \hat{B}(l)\right)-\mathbb{P}^{z_{1}}\left(X_{T_{l}}=w \mid \hat{B}(l)\right)\right|
\end{aligned}
$$

and breaking the last sum into $l \leq N^{2} / 2$ and $l>N^{2} / 2$, then controlling the former using Lemma 4.1 and the latter using (4.16), we get part (3) of the lemma. Part (2) follows from the exact same calculations using (4.4). Part (4) follows from (4.5) similarly. To see (5) and (6), we run the same calculation with one main difference: When we do the 
calculation equivalent to (4.10), instead of (4.5), we use (4.6) (for (5)) or (4.7) (for (6)). We then get a factor of $k^{(-2-d) / 2}$ instead of $k^{(-1-d) / 2}$ (this is because the inequalities (4.6) and (4.7) give an extra factor of $\sqrt{k}$ ), and we continue to carry this factor of $\sqrt{k}$ all the way through.

Proof of Claim 4.3. The proof is a standard Fourier calculation, and therefore we do not give complete details. By the assumptions, the characteristic function $\chi$ of $Y_{2}$ has period $2 \pi$ in every coordinate. In addition, since $\mathbf{P}\left(Y_{2}=v\right)>0$ and $\mathbf{P}\left(Y_{2}=v+e_{i}\right)>0$ for $i=1, \ldots, d$, there exist $D>0$ and $\delta>0$ such that

- $|\chi(x)|<1-D$ for every $x \in[-\pi, \pi]^{d}$ such that $\|x\| \geq \delta$, and

- $|\chi(x)|<1-D\|x\|^{2}$ for every $x$ such that $\|x\|<\delta$.

Let $S^{\prime}=\sum_{k=2}^{n} Y_{k}$ and let $A=\{x:\|x\|<\delta\}$. Now, to see (4.4), we note that

$$
\begin{aligned}
\mathbf{P}\left(S^{\prime}=z\right) & =\frac{1}{(2 \pi)^{d}} \int_{[-\pi, \pi]^{d}} e^{-i\langle x, z\rangle} \chi(x)^{n-1} d x \\
& \leq \int_{[-\pi, \pi]^{d}}|\chi(x)|^{n-1} d x \leq(1-D)^{n-1}+\int_{A}\left(1-D\|x\|^{2}\right)^{n-1} d x \leq C n^{-d / 2},
\end{aligned}
$$

and convolution with the distribution of $Y_{1}$ only decreases the supremum.

To see (4.5), we see that

$$
\begin{aligned}
\left|\mathbf{P}\left(S^{\prime}=z\right)-\mathbf{P}\left(S^{\prime}=z+e_{i}\right)\right| & =\frac{1}{(2 \pi)^{d}}\left|\int_{[-\pi, \pi]^{d}}\left(e^{-i\langle x, z\rangle}-e^{-i\left\langle x, z+e_{i}\right\rangle}\right) \chi(x)^{n-1} d x\right| \\
& \leq \frac{1}{(2 \pi)^{d}} \int_{[-\pi, \pi]^{d}}\left|e^{-i\langle x, z\rangle}-e^{-i\left\langle x, z+e_{i}\right\rangle}\right||\chi(x)|^{n-1} d x .
\end{aligned}
$$

Note that $\left|e^{-i\langle x, z\rangle}-e^{-i\left\langle x, z+e_{i}\right\rangle}\right| \leq\left|\left\langle x, e_{i}\right\rangle\right|$. Therefore,

$$
\begin{aligned}
\left|\mathbf{P}\left(S^{\prime}=z+e_{1}\right)-\mathbf{P}\left(S^{\prime}=z\right)\right| & \leq \frac{1}{(2 \pi)^{d}} \int_{[-\pi, \pi]^{d}}|\chi(x)|^{n-1}\left\langle x, e_{1}\right\rangle d x \\
& \leq(1-D)^{n-1}+C \int_{A} e^{-D n\|x\|^{2}}\left\langle x, e_{1}\right\rangle d x,
\end{aligned}
$$

and substituting $y=x \sqrt{n}$ we get $d x=n^{-d / 2} d y$ and $\left\langle x, e_{1}\right\rangle=n^{-1 / 2}\left\langle y, e_{1}\right\rangle$, so the last integral is bounded by

$$
\begin{aligned}
C n^{-d / 2-1 / 2} \int_{\sqrt{n} A} e^{-D\|y\|^{2}}\left\langle y, e_{1}\right\rangle d y & \leq C n^{-d / 2-1 / 2} \int_{\mathbb{R}^{d}} e^{-D\|y\|^{2}}\left\langle y, e_{1}\right\rangle d y \\
& =O\left(n^{(-d-1) / 2}\right) .
\end{aligned}
$$

To see (4.6), we note that $\left|e^{-i\left\langle x, z+e_{1}\right\rangle}+e^{-i\left\langle x, z-e_{1}\right\rangle}-2 e^{-i\langle x, z\rangle}\right| \leq\left\langle x, e_{1}\right\rangle^{2}$ for every $z \in \mathbb{Z}^{d}$. Then,

$$
\begin{aligned}
& \left|\mathbf{P}\left(S^{\prime}=z+e_{1}\right)+\mathbf{P}\left(S^{\prime}=z-e_{1}\right)-2 \mathbf{P}\left(S^{\prime}=z\right)\right| \\
& \quad \leq \frac{1}{(2 \pi)^{d}} \int_{[-\pi . \pi]^{d}}|\chi(x)|^{n-1}\left\langle x, e_{1}\right\rangle^{2} d x \leq(1-D)^{n-1}+C \int_{A} e^{-D n\|x\|^{2}}\left\langle x, e_{1}\right\rangle^{2} d x,
\end{aligned}
$$


and again substituting $y=x \sqrt{n}$, we still get $d x=n^{-d / 2} d y$ but this time $\left\langle x, e_{1}\right\rangle^{2}=$ $n^{-1}\left\langle y, e_{1}\right\rangle^{2}$. Therefore, this time the last integral is bounded by

$$
\begin{aligned}
C n^{-d / 2-1} \int_{\sqrt{n} A} e^{-D\|y\|^{2}}\left\langle y, e_{1}\right\rangle^{2} d y & \leq C n^{-d / 2-1} \int_{\mathbb{R}^{d}} e^{-D\|y\|^{2}}\left\langle y, e_{1}\right\rangle^{2} d y \\
& =O\left(n^{(-d-2) / 2}\right) .
\end{aligned}
$$

The way to see (4.7) is similar-this time what we need to notice is that $\mid e^{-i\langle x, z\rangle}-$ $e^{-i\left\langle x, z+e_{1}\right\rangle}-e^{-i\left\langle x, z+e_{2}\right\rangle}+e^{-i\left\langle x, z+e_{1}+e_{2}\right\rangle} \mid \leq\left\langle x, e_{1}\right\rangle \cdot\left\langle x, e_{2}\right\rangle$ and that when we substitute $y=\sqrt{n} x$ we get $\left\langle x, e_{1}\right\rangle \cdot\left\langle x, e_{2}\right\rangle=n^{-1}\left\langle y, e_{1}\right\rangle \cdot\left\langle y, e_{2}\right\rangle$.

Lemma 4.4. Assume the assumptions 1-3 from page 136. Let $\left\{X_{n}\right\}$ be a RWRE starting at the origin. Let $\sigma^{2}$ be the (annealed) covariance matrix of $X_{\tau_{2}}-X_{\tau_{1}}$, and let $U$ be the expectation of $X_{\tau_{2}}-X_{\tau_{1}}$. Let $\Sigma$ be the inverse matrix of $\sigma^{2}$. Let $\bar{U}=\mathbb{E}\left(X_{\left.T_{\partial \mathcal{P}(0, N)}\right)}\right)$. Fix $a>0$. There exists a constant $c$ such that for every $x \in \partial^{+} \mathcal{P}(0, N)$, if

$$
(x-\bar{U})^{T} \Sigma(x-\bar{U})<a \cdot \frac{N^{2}}{\left\langle U, e_{1}\right\rangle},
$$

then

$$
\mathbb{P}\left(X_{T_{\partial \mathcal{P}(0, N)}}=x\right)>c N^{1-d} e^{-3 a} .
$$

Proof. The proof is very similar to that of Lemma 4.2, but slightly simpler. We continue to use the notations $B(l, k), B(l)$ and $\hat{B}(l)$. Let $\delta=\delta(a)$ be a small number. By the local limit theorem (see e.g. [5]), for $l>N^{2} / 2$ and every $y \in \mathcal{P}(0, N)$ such that $\left\langle y, e_{1}\right\rangle=l$,

$$
\mathbb{P}\left(Z_{l}=y ; B(l, k)\right) \geq(2 \pi \beta)^{-1 / 2} k^{-d / 2}\left(e^{-(y-U k)^{T} \Sigma(y-U k) / 2 k}-\delta\right)
$$

where $\beta$ is the determinant of $\sigma^{2}$.

Fix $l=N^{2}$ and let $M=N^{2} /\left\langle U, e_{1}\right\rangle$. Then, using (4.17), for $x \in \partial^{+} \mathcal{P}(0, N)$,

$$
\begin{aligned}
\mathbb{P}\left(Z_{N^{2}}=x\right) & \geq \mathbb{P}\left(Z_{N^{2}}=x ; B\left(N^{2}\right)\right) \geq \sum_{k=\lceil M-\sqrt{M}\rceil}^{\lceil M+\sqrt{M}\rceil} \mathbb{P}\left(Z_{N^{2}}=x ; B\left(N^{2}, k\right)\right) \\
& \geq(\pi \beta)^{-1 / 2} M^{-d / 2} \sum_{k=\lceil M-\sqrt{M}\rceil}^{\lceil M+\sqrt{M}\rceil}\left(e^{-\frac{(x-U k)^{T} \Sigma(x-U k)}{M-\sqrt{M}}}-\delta\right) \\
& \geq(\pi \beta)^{-1 / 2} M^{-(d-1) / 2}\left(e^{-\frac{(x-\bar{U})^{T} \Sigma(x-\bar{U})}{M-\sqrt{M}}-\frac{(\sqrt{M} U)^{T} \Sigma(\sqrt{M} U)}{M-\sqrt{M}}}-\delta\right) \\
& \geq c N^{1-d} e^{-3 a} .
\end{aligned}
$$

\subsection{Quenched exit estimates}

In this subsection we show that with very high probability the quenched exit distribution from a basic block is similar to the annealed one. This is the only part of the paper that requires the high dimension assumption. 
The goal of this subsection is the following proposition:

Proposition 4.5. Assume the assumptions 1-3 from page 136. Fix $0<\theta \leq 1$. There exists an event $G(N)=G(\theta, N) \subseteq \Omega$ such that $P(G(N))=1-N^{-\xi(1)}$ and such that for all $\omega \in G(N)$ :

(1) For every $z \in \tilde{\mathcal{P}}(0, N)$,

$$
P_{\omega}^{z}\left(T_{\partial \mathcal{P}(0, N)} \neq T_{\partial+\mathcal{P}(0, N)}\right)=N^{-\xi(1)} .
$$

(2) For every $z \in \tilde{\mathcal{P}}(0, N)$,

$$
\left\|E_{\omega}^{z}\left[X_{T_{\partial \mathcal{P}(0, N)}}\right]-\mathbb{E}^{z}\left[X_{\left.T_{\partial \mathcal{P}(0, N)}\right] \|}\right]\right\| R_{3}(N) .
$$

(3) For every $z \in \tilde{\mathcal{P}}(0, N)$ and every $(d-1$-dimensional $)$ cube $Q \subseteq \partial^{+} \mathcal{P}(0, N)$ of side length $\left[N^{\theta}\right]$,

$$
\left|P_{\omega}^{z}\left[X_{T_{\partial \mathcal{P}(0, N)}} \in Q\right]-\mathbb{P}^{z}\left[X_{T_{\partial \mathcal{P}(0, N)}} \in Q\right]\right|<N^{(\theta-1)(d-1)-\theta \frac{d-1}{d+1}} .
$$

From Proposition 4.5 we get the following corollary:

Corollary 4.6. Assume the assumptions $1-3$ from page 136. Fix $\theta<1 / 2$ and let $G(N)$ be as in Proposition 4.5. Let $\omega \in G(N)$ and $z \in \tilde{\mathcal{P}}(0, N)$. Let $D=D(\omega, z)$ be the quenched exit distribution from $\mathcal{P}(0, N)$, and let $\bar{D}=\bar{D}(\omega, z)$ be $D$ conditioned on $\partial^{+} \mathcal{P}(0, N)$. Let $\mathbb{D}=\mathbb{D}(z)$ be the annealed exit distribution, and $\overline{\mathbb{D}}$ the annealed exit distribution conditioned on $\partial^{+} \mathcal{P}(0, N)$. Then:

(1) $D\left(\partial^{+} \mathcal{P}(0, N)\right)=1-N^{-\xi(1)}$.

(2) If $X \sim \bar{D}$, then $X$ can be written as $X=Y+Z$, where $\|Z\| \leq(d+1) N^{\theta}$ a.s. and $Y \sim \overline{\mathbb{D}}+D_{2}$, where $D_{2}$ is a signed measure such that:

(a) $\left\|D_{2}\right\|:=\sum_{x}\left|D_{2}(x)\right| \leq \lambda=N^{-\theta(d-1) / 2(d+1)}$.

(b) $\sum_{x} D_{2}(x)=0$.

(c) $\sum_{x} x D_{2}(x)=0$.

(d) $\sum_{x}\left|D_{2}(x)\right|\left\|x-E_{\overline{\mathbb{D}}}\right\|_{1}^{2} \leq \lambda N^{2}$, where $E_{\overline{\mathbb{D}}}$, a vector in $\mathbb{R}^{d}$, is the expectation of the probability distribution $\overline{\mathbb{D}}$.

Proof. Part (1) is trivial, and therefore we will prove (2). Partition $\partial^{+} \mathcal{P}(0, N)$ into disjoint cubes $Q_{1}, \ldots, Q_{n}$ of side length $N^{\theta}$. We get $n=R_{5}(N)^{d-1} N^{(d-1)(1-\theta)}$ such cubes. For every $1 \leq k \leq n$,

$$
\left|\bar{D}\left(Q_{k}\right)-\overline{\mathbb{D}}\left(Q_{k}\right)\right| \leq N^{(\theta-1)(d-1)-\theta \frac{d-1}{d+1}} .
$$

We define $Y^{\prime}$ as follows: For every $k$, we take $Y^{\prime}$ to be in $Q_{k}$ whenever $X \in Q_{k}$. Conditioned on the event $Y^{\prime} \in Q_{k}$, we take $Y^{\prime}$ to be independent of $X$, with

$$
\mathbf{P}\left(Y^{\prime}=x \mid Y^{\prime} \in Q_{k}\right)=\overline{\mathbb{D}}(x) / \overline{\mathbb{D}}\left(Q_{k}\right)
$$

for every $x \in Q_{k}$. Then clearly $\left\|X-Y^{\prime}\right\|<d N^{\theta}$. Therefore, $\left\|E\left(Y^{\prime}\right)-E(X)\right\|<d N^{\theta}$. By (4.18), $\left\|E(X)-E_{\overline{\mathbb{D}}}\right\| \leq R_{3}(N)$ and thus $\left\|E\left(Y^{\prime}\right)-E_{\overline{\mathbb{D}}}\right\|<(d+1) N^{\theta}$. Then there 
exists a variable $U$, independent of $Y^{\prime}$ and $X$, such that $\|U\|<(d+1) N^{\theta}+1$ and $E\left(Y^{\prime}+U\right)=E_{\overline{\mathbb{D}}}$. Define $Y=Y^{\prime}+U$. Then (2)(b) and (2)(c) are immediate.

To see (2)(a), we first note that

$$
\mathbf{P}(Y=x)=\sum_{u:\|u\|<(d+1) N^{\theta}+1} \mathbf{P}(U=u) \mathbf{P}\left(Y^{\prime}=x-u\right) .
$$

Therefore,

$$
\begin{aligned}
\sum_{x} \mid \mathbf{P}(Y=x) & -\overline{\mathbb{D}}(x) \mid \\
& \leq \sum_{u:\|u\|<(d+1) N^{\theta}+1} \mathbf{P}(U=u) \sum_{x}\left|\mathbf{P}\left(Y^{\prime}=x-u\right)-\overline{\mathbb{D}}(x)\right| .
\end{aligned}
$$

By Lemma 4.2(3), for all $x$ and $u$ such that $\|u\|<(d+1) N^{\theta}+1$,

$$
|\overline{\mathbb{D}}(x-u)-\overline{\mathbb{D}}(x)| \leq C(d+1) N^{\theta} \cdot N^{-d}=C(d+1) N^{\theta-d} .
$$

Therefore, with $D_{2}$ as defined in (2),

$$
\begin{aligned}
\sum_{x}\left|D_{2}(x)\right| & =\sum_{x}|\mathbf{P}(Y=x)-\overline{\mathbb{D}}(x)| \leq \sum_{x}\left(\left|\mathbf{P}\left(Y^{\prime}=x\right)-\overline{\mathbb{D}}(x)\right|+C(d+1) N^{\theta-d}\right) \\
& =\left(\sum_{k=1}^{n}\left|\bar{D}\left(Q_{k}\right)-\overline{\mathbb{D}}\left(Q_{k}\right)\right|\right)+R_{5}(N)^{d-1} N^{d-1} \cdot C(d+1) N^{\theta-d} \\
& \leq C(d+1) R_{5}(N)^{d-1} N^{\theta-1}+R_{5}(N)^{d-1} N^{(d-1)(1-\theta)} \cdot N^{(\theta-1)(d-1)-\theta \frac{d-1}{d+1}} \\
& =R_{5}(N)^{d-1}\left(C(d+1) N^{\theta-1}+N^{-\theta \frac{d-1}{d+1}}\right) \leq R_{6}(N) N^{-\theta \frac{d-1}{d+1}}<\lambda .
\end{aligned}
$$

To see $(2)(\mathrm{d})$, note that $\left\|x-E_{\overline{\mathbb{D}}}\right\|_{1} \leq d N R_{5}(N)$ for every $x$ in the support of $D_{2}$. Therefore,

$$
\begin{aligned}
\sum_{x}\left|D_{2}(x)\right|\left\|x-E_{\overline{\mathbb{D}}}\right\|_{1}^{2} & \leq d^{2} R_{5}(N)^{2} N^{2} \sum_{x}\left|D_{2}(x)\right| \\
& \leq d^{2} R_{5}(N)^{2} N^{2} \cdot R_{6}(N) N^{-\theta \frac{d-1}{d+1}} \leq \lambda N^{2} .
\end{aligned}
$$

Corollary 4.6 can be formulated slightly differently in the language of couplings. We need a definition.

Definition 11. For two probability measures $\mu_{1}$ and $\mu_{2}$ on $\mathbb{Z}^{d}$, and for $\lambda<1$ and $k \in \mathbb{N}$, we say that $\mu_{2}$ is $(\lambda, k)$-close to $\mu_{1}$ if there exists a joint distribution ("coupling") $\mu$ of three random variables $Z_{1}, Z_{2}$ and $Z_{0}$ such that:

(1) $Z_{1} \sim \mu_{1}$ and $Z_{2} \sim \mu_{2}$.

(2) $\mu\left(Z_{1} \neq Z_{0}\right) \leq \lambda$.

(3) $\mu\left(\left\|Z_{0}-Z_{2}\right\|<k\right)=1$.

(4) $\sum_{x} x\left[\mu\left(Z_{1}=x\right)-\mu\left(Z_{0}=x\right)\right]=E_{\mu}\left(Z_{1}\right)-E_{\mu}\left(Z_{0}\right)=0$.

(5) $\sum_{x}\left|\mu\left(Z_{1}=x\right)-\mu\left(Z_{0}=x\right)\right|\left\|x-E_{\mu}\left(Z_{1}\right)\right\|_{1}^{2} \leq \lambda \operatorname{var}\left(Z_{1}\right)$. 
Using Definition 11, Corollary 4.6(2) can be formulated as saying that if $\omega \in G(N)$, then $\bar{D}$ is $\left(N^{-\theta(d-1) / 2(d+1)},(d+1) N^{\theta}\right)$-close to $\overline{\mathbb{D}}$. (We need to see that the variance of a $\overline{\mathbb{D}}$ distributed variable is at least at the order of magnitude of $N^{2}$. This follows, e.g., from the annealed lower bound in Lemma 4.4.)

The following claim is immediate and useful.

Claim 4.7. In the language of Definition 11 , the distribution of $Z_{0}$ is $(\lambda, 0)$-close to $\mu_{1}$.

We now proceed to proving Proposition 4.5. We start with a version of Azuma's inequality. Let $\left\{M_{k}\right\}_{k=1}^{n}$ be a zero mean martingale with respect to a filtration $\left\{\mathcal{F}_{k}\right\}_{k=1}^{n}$ on the sample space $\Omega$. For simplicity we denote $M_{0}=0$ and $\mathcal{F}_{0}=\{\emptyset, \Omega\}$. For $k=1, \ldots, n$, let $D_{k}=M_{k}-M_{k-1}$. Set

$$
U_{k}=\operatorname{ess} \sup \left(\left|D_{k}\right| \mid \mathcal{F}_{k-1}\right)=\lim _{p \rightarrow \infty}\left[E\left(\left|D_{k}\right|^{p} \mid \mathcal{F}_{k-1}\right)\right]^{1 / p}
$$

and define the essential variance of the martingale to be

$$
U:=\operatorname{ess} \sup \left(\sum_{k=1}^{n} U_{k}^{2}\right)
$$

Lemma 4.8. For every $K$,

$$
\mathbf{P}\left(\left|M_{n}\right|>K\right) \leq 2 e^{-K^{2} / 2 U} .
$$

Proof. The proof is similar to that of Azuma's inequality: First we show that for every $k$,

$$
\mathbf{E}\left(e^{\sum_{j=k}^{n} D_{j}} \mid \mathcal{F}_{k-1}\right) \leq e^{\frac{1}{2} \operatorname{ess} \sup \left(\sum_{j=k}^{n} U_{j}^{2} \mid \mathcal{F}_{k-1}\right)} .
$$

Indeed, (4.21) is clear for $k=n$, and assuming it holds for $k+1$, we get

$$
\begin{aligned}
\mathbf{E}\left(e^{\sum_{j=k}^{n} D_{j}} \mid \mathcal{F}_{k-1}\right)=\mathbf{E}\left(e^{D_{k}} \mathbf{E}\left(e^{\sum_{j=k+1}^{n} D_{j}} \mid \mathcal{F}_{k}\right) \mid \mathcal{F}_{k-1}\right) \\
\quad \leq \mathbf{E}\left(e^{D_{k}} e^{\frac{1}{2} \operatorname{ess} \sup \left(\sum_{j=k+1}^{n} U_{j}^{2} \mid \mathcal{F}_{k}\right)} \mid \mathcal{F}_{k-1}\right) \leq \mathbf{E}\left(e^{D_{k}} e^{\frac{1}{2} \operatorname{ess} \sup \left(\sum_{j=k+1}^{n} U_{j}^{2} \mid \mathcal{F}_{k-1}\right)} \mid \mathcal{F}_{k-1}\right) \\
\quad=e^{\frac{1}{2} \operatorname{ess} \sup \left(\sum_{j=k+1}^{n} U_{j}^{2} \mid \mathcal{F}_{k-1}\right)} \mathbf{E}\left(e^{D_{k}} \mid \mathcal{F}_{k-1}\right) \leq e^{\frac{1}{2} \operatorname{ess} \sup \left(\sum_{j=k+1}^{n} U_{j}^{2} \mid \mathcal{F}_{k-1}\right)} e^{\frac{1}{2} U_{k}^{2}} \\
\quad=e^{\frac{1}{2} \operatorname{ess} \sup \left(\sum_{j=k}^{n} U_{j}^{2} \mid \mathcal{F}_{k-1}\right)}
\end{aligned}
$$

For $k=0$ this gives $\mathbf{E}\left(e^{M_{n}}\right) \leq e^{\frac{1}{2} U}$, and for every $\lambda$,

$$
\mathbf{E}\left(e^{\lambda M_{n}}\right) \leq e^{\frac{1}{2} \lambda^{2} U} .
$$

Using Markov's inequality once with $\lambda=K / U$ and once with $\lambda=-K / U$ gives the desired result.

Next we discuss the intersection structure of two independent walks in the same environment. 
Lemma 4.9. Assume the assumptions $1-3$ from page 136. Let $X^{(1)}:=\left\{X_{n}^{(1)}\right\}$ and $X^{(2)}$ $:=\left\{X_{n}^{(2)}\right\}$ be two independent random walks running in the same environment $\omega$. Let $\left[X^{(i)}\right]$ be the set of points visited by $\left\{X_{n}^{(i)}\right\}$. Then there exists $C$ such that for every $n$,

$E\left[P_{\omega, \omega}\left(\left\{\left|\left[X^{(1)}\right] \cap\left[X^{(2)}\right] \cap \mathcal{P}(0, N)\right|>n R_{1}(N)^{d}\right\} \cap A_{N}\left(X^{(1)}\right) \cap A_{N}\left(X^{(2)}\right)\right)\right]<e^{-C n}$.

Proof. Let $k \geq 0$ be such that $k+R_{1}(N)<N$. Then from the definition of the event $A_{N}$, for a random walk $X=\left\{X_{n}\right\}$,

$$
\mathbf{1}_{A_{N}(X)} \cdot\left|\left\{x: x \in[X], k<\left\langle x, e_{1}\right\rangle<k+R_{1}(N)\right\}\right|<2^{d} R_{1}(N)^{d} .
$$

For every $k$, let $Q_{k}^{-}=\mathcal{P}(0, N) \cap\left\{x:\left\langle x, e_{1}\right\rangle<k R_{1}(N)\right\}$ and $Q_{k}^{+}=\mathcal{P}(0, N) \cap\{x$ : $\left.\left\langle x, e_{1}\right\rangle \geq k R_{1}(N)\right\}$. In addition, let $\hat{A}_{N}=A_{N}\left(X^{(1)}\right) \cap A_{N}\left(X^{(2)}\right)$. Using Propositions 3.1, 3.4 and 3.7 of [1], as well as uniform ellipticity, and again recalling the definition of $A_{N}$, we see that there exists $\rho>0$ such that for every $k$,

$$
E\left[P_{\omega, \omega}\left(\left\{\left[X^{(1)}\right] \cap\left[X^{(2)}\right] \cap Q_{k+1}^{+}=\emptyset\right\} \mid \hat{A}_{N} ;\left[X^{(1)}\right] \cap Q_{k}^{-} ;\left[X^{(2)}\right] \cap Q_{k}^{-}\right)\right]>\rho .
$$

Remark. As stated in [1], Propositions 3.1, 3.4 and 3.7 of [1] require moment assumptions on the regeneration times. However, examining their proofs shows that all they need are moment assumptions on the number of sites visited before $\tau_{1}$, and these are satisfied by Lemma 4.1.

Now, let

$$
\begin{aligned}
J^{(\text {even })} & =\left\{k: k \text { is even and }\left[X^{(1)}\right] \cap\left[X^{(2)}\right] \cap Q_{k}^{+} \cap Q_{k+1}^{-} \neq \emptyset\right\}, \\
J^{(\text {odd })} & =\left\{k: k \text { is odd and }\left[X^{(1)}\right] \cap\left[X^{(2)}\right] \cap Q_{k}^{+} \cap Q_{k+1}^{-} \neq \emptyset\right\} .
\end{aligned}
$$

Then, by (4.23), conditioned on $\hat{A}_{N}$, both $J^{\text {(even) }}$ and $J^{\text {(odd) }}$ are dominated by a geometric variable with parameter $\rho$. The lemma now follows when we remember that by (4.22),

$$
\mathbf{1}_{\hat{A}_{N}} \cdot\left|\left[X^{(1)}\right] \cap\left[X^{(2)}\right] \cap \mathcal{P}(0, N)\right| \leq 2^{d} R_{1}(N)^{d}\left(J^{(\text {even })}+J^{(\text {odd })}\right) .
$$

As a corollary we get the following estimate:

Lemma 4.10. Assume the assumptions 1-3 from page 136. With the same notation as in Lemma 4.9,

$P\left[\omega: E_{\omega, \omega}\left(\left|\left[X^{(1)}\right] \cap\left[X^{(2)}\right] \cap \mathcal{P}(0, N)\right| \cdot \mathbf{1}_{A_{N}\left(X^{(1)}\right) \cap A_{N}\left(X^{(2)}\right)}\right) \geq R_{2}(N)\right]=N^{-\xi(1)}$.

Let $J(N) \subseteq \Omega$ be the event that for every starting point $z$ in the middle third of the block,

$$
E_{\omega, \omega}^{z, z}\left(\left|\left[X^{(1)}\right] \cap\left[X^{(2)}\right] \cap \mathcal{P}(0, N)\right| \cdot \mathbf{1}_{A_{N}\left(X^{(1)}\right) \cap A_{N}\left(X^{(2)}\right)}\right) \leq R_{2}(N) .
$$

Then, by Lemma 4.10, $P(J(N))=1-N^{-\xi(1)}$.

Fix $z \in \tilde{\mathcal{P}}(0, N)$. For every $\omega$ and $x \in \mathcal{P}(0, N)$, we let

$$
H^{z}(\omega, x):=P_{\omega}^{z}\left(x \in[X] \text { and } A_{N}\left(\left\{X_{n}\right\}\right)\right)
$$

be the hitting probability of $x$. Then for $\omega \in J(N)$,

$$
\sum_{x \in \mathcal{P}(0, N)}\left(H^{z}(\omega, x)\right)^{2} \leq R_{2}(N)
$$


Lemma 4.11. Assume the assumptions 1-3 from page 136. There exists an event $K(N)$ $\subseteq \Omega$ such that $P(K(N))=1-N^{-\xi(1)}$ and for every $\omega \in K(N)$ and $z \in \tilde{\mathcal{P}}(0, N)$,

$$
\left\|E_{\omega}^{z}\left[X_{T_{\partial \mathcal{P}(0, N)}}\right]-\mathbb{E}^{z}\left[X_{T_{\partial \mathcal{P}(0, N)}}\right]\right\| \leq R_{3}(N) .
$$

Proof. Define

$$
\begin{aligned}
& U(\omega, z):=\| E_{\omega}^{z}\left[X_{T_{\partial \mathcal{P}(0, N)}} \cdot \mathbf{1}_{A_{N}} \cdot \mathbf{1}_{T_{\partial \mathcal{P}(0, N)}=T_{\partial}+\mathcal{P}(0, N)}\right] \\
&-\mathbb{E}^{z}\left[X_{T_{\partial \mathcal{P}(0, N)}} \cdot \mathbf{1}_{A_{N}} \cdot \mathbf{1}_{T_{\partial \mathcal{P}(0, N)}=T_{\partial}+\mathcal{P}(0, N)} \mid J(N)\right] \| .
\end{aligned}
$$

It is sufficient to show that for a large enough set of $\omega$ 's,

$$
U(\omega, z) \leq R_{2}(N)^{2 d+2} .
$$

(4.27) is sufficient, because for a set $M$ of environments of measure $1-N^{-\xi(1)}$, for every $\omega \in M$ we have $P_{\omega}^{z}\left(T_{\partial \mathcal{P}(0, N)}=T_{\partial+\mathcal{P}(0, N))}\right)=N^{-\xi(1)}$. Since $\left\|X_{T_{\partial \mathcal{P}(0, N)}}\right\|_{\infty}<C N^{2}$, on every $\omega \in M$ the contribution of the event $\left.\left\{T_{\partial \mathcal{P}(0, N)} \neq T_{\partial}+\mathcal{P}(0, N)\right)\right\}$ to the expectation of $X_{T_{\partial \mathcal{P}(0, N)}}$ is bounded by 1 .

To show (4.27), we order the vertices in $\mathcal{P}(0, N)$ lexicographically, $x_{1}, x_{2}, \ldots$, with the first coordinate being the most significant. Let $\mathcal{F}_{n}$ be the $\sigma$-algebra on the sample space $(J(N) \subseteq \Omega, P(\cdot \mid J(N)))$ which is determined by $\left.\omega\right|_{x_{1}, \ldots, x_{n}}$ and let $\left\{M_{k}\right\}$ be the martingale

$$
M_{k}:=E\left[E_{\omega}\left[X_{T_{\partial \mathcal{P}(0, N)}} \cdot \mathbf{1}_{A_{N}} \cdot \mathbf{1}_{T_{\partial \mathcal{P}(0, N)}=T_{\partial}+\mathcal{P}(0, N)}\right] \mid \mathcal{F}_{k}\right] .
$$

Next we calculate ess $\sup \left(M_{k}-M_{k-1} \mid \mathcal{F}_{k-1}\right)$. The argument is similar to the one used in [1], which is based on ideas from [2]. Let

$$
B(x):=\left\{y:\left\langle y, e_{1}\right\rangle=\left\langle x, e_{1}\right\rangle-1 \text { and }\|y-x\| \leq R_{1}(N)^{2}\right\} .
$$

Note that if $x$ is visited and $A_{N}$ holds, then the first visit to the layer

$$
H(x):=\left\{y:\left\langle y, e_{1}\right\rangle=\left\langle x, e_{1}\right\rangle-1\right\}
$$

is in $B(x)$. Therefore,

$$
\begin{aligned}
U_{k} & =\operatorname{ess} \sup \left(M_{k}-M_{k-1} \mid \mathcal{F}_{k-1}\right) \leq R(N)^{2} \mathrm{P}\left(x_{k} \in[X] \mid \mathcal{F}_{k-1}\right) \\
& \leq R(N)^{2} \sum_{y \in B\left(x_{k}\right)} \mathrm{P}\left(X_{T_{H\left(x_{k}\right)}}=y \mid \mathcal{F}_{k-1}\right)=R(N)^{2} \sum_{y \in B\left(x_{k}\right)} P_{\omega}\left(X_{T_{H\left(x_{k}\right)}}=y\right) \\
& \leq R(N)^{2} \sum_{y \in B\left(x_{k}\right)} P_{\omega}(y \in[X])
\end{aligned}
$$

where the first inequality follows from the fact that the regeneration containing $x_{k}$ is of size no more than $R(N)^{2}$, and after this regeneration the distribution of the walk is the 
annealed distribution. Remembering that $\left|B\left(x_{k}\right)\right| \leq 2^{d} R_{2}(N)^{d}$ and that every $y$ is in $B(x)$ for at most $2^{d} R_{2}(N)^{d}$ points $x$, we have

$$
\begin{aligned}
\sum_{k=1}^{n} U_{k}^{2} & \leq \sum_{k=1}^{n} R(N)^{4}\left[\sum_{y \in B\left(x_{k}\right)} P_{\omega}(y \in[X])\right]^{2} \\
& \leq 2^{d} R_{2}(N)^{d} R(N)^{4} \sum_{k=1}^{n} \sum_{y \in B\left(x_{k}\right)} P_{\omega}(y \in[X])^{2} \\
& \leq 2^{2 d} R_{2}(N)^{2 d} R(N)^{4} \sum_{y \in \mathcal{P}(0, N)} H^{2}(\omega, y) \leq 2^{2 d} R_{2}(N)^{2 d} R(N)^{4} \cdot R_{2}(N) \\
& \leq R_{2}(N)^{2 d+2} .
\end{aligned}
$$

Therefore, by Lemma 4.8,

$$
P\left(\omega: U(\omega)>R_{2}(N)^{2 d+2} \mid J(N)\right)<2 e^{-\frac{R_{2}(N)^{4 d+4}}{2 R_{2}(N)^{2 d+2}}}=N^{-\xi(1)} .
$$

(4.27) follows.

We now estimate the quenched exit distribution from $\mathcal{P}(0, N)$. Fix a starting point for the walk $z \in \tilde{\mathcal{P}}(0, N)$. We start with the following lemma. Recall that for every $k$, we define $H_{k}$ to be the hyperplane $H_{k}=\left\{v \in \mathbb{Z}^{d}:\left\langle v, e_{1}\right\rangle=k\right\}$.

Lemma 4.12. Assume the assumptions $1-3$ from page 136. Fix $0<\theta \leq 1$. Let $B^{\theta}(N) \subseteq$ $\Omega$ be the event that for every $\frac{2}{5} N^{2} \leq M \leq N^{2}$ and every $(d-1$-dimensional $)$ cube $Q$ of side length $N^{\theta}$ which is contained in $H_{M}$,

$$
\left|P_{\omega}^{z}\left(X_{T_{M}} \in Q ; A_{N}\right)-\mathbb{P}^{z}\left(X_{T_{M}} \in Q ; A_{N}\right)\right| \leq N^{(\theta-1)(d-1)} .
$$

Then for $\theta>(d-1) / d$,

$$
P\left(B^{\theta}(N)\right)=1-N^{-\xi(1)} .
$$

Proof. Fix $\theta$, and let $(d-1) / d<\theta^{\prime}<\theta$. Let

$$
V=\left[N^{2 \theta^{\prime}}\right]
$$

Fix $\frac{2}{5} N^{2} \leq M \leq N^{2}$. Let $v \in H_{M+V}$, and let $\mathcal{G}$ be the $\sigma$-algebra that is determined by the configuration on

$$
\mathcal{P}^{M}(0, N)=\mathcal{P}(0, N) \cap\left\{x:\left\langle x, e_{1}\right\rangle \leq M\right\} .
$$

We are interested in the quantity

$$
J^{(M)}(v)=E\left[P_{\omega}\left(X_{T_{M+V}}=v ; A_{N}\right) \mid \mathcal{G}\right] .
$$

Similar to the proof of Lemma 4.11, we let $\left\{x_{i}\right\}_{i=1}^{n}$ be a lexicographic ordering of the vertices in $\mathcal{P}^{M}(0, N)$, and let $\left\{\mathcal{F}_{i}\right\}$ be the $\sigma$-algebra on $J(N)$ which is determined by $\left.\omega\right|_{x_{1}, \ldots, x_{i}}$. 


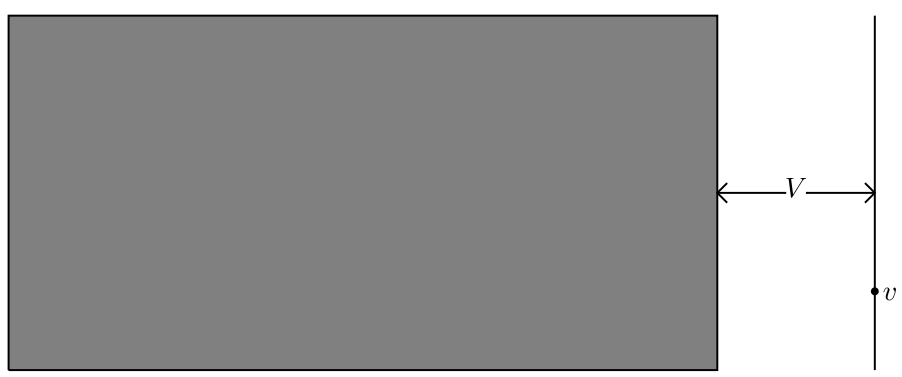

Fig. 3. The quantity $J^{(M)}(v)$ is the probability of hitting the point $v$, conditioned on the environment in the shaded area, and averaged over the environment elsewhere.

We consider the martingale $M_{i}=E\left[P_{\omega}\left(X_{T_{M+V}}=v ; A_{N}\right) \mid \mathcal{F}_{i}\right]$. In order to use Lemma 4.8, we will need to bound $U_{i}=\operatorname{ess} \sup \left(M_{i}-M_{i-1} \mid \mathcal{F}_{i-1}\right)$. Remember that $x_{i}$ is the vertex such that $\omega_{x_{i}}$ is measurable with respect to $\mathcal{F}_{i}$ but not with respect to $\mathcal{F}_{i-1}$. Then we claim that

$$
U_{i} \leq C R(N) E\left[P_{\omega}\left(x_{i} \text { is hit }\right) \mid \mathcal{F}_{i-1}\right] V^{-d / 2} .
$$

We now show the main estimate (4.30). Let $\omega^{\prime}$ be an environment that agrees with $\omega$ everywhere except possibly at $x_{i}$. We let $\mathbf{P}$ be the distribution of a walk that follows the law $\omega$ on $\left\{x_{k}: k \leq i\right\}$ and the annealed distribution on $\mathbb{Z}^{d} \backslash\left\{x_{k}: k \leq i\right\}$. Equivalently, let $\mathbf{P}^{\prime}$ be the distribution of a walk that follows the law $\omega^{\prime}$ on $\left\{x_{k}: k \leq i\right\}$ and the annealed distribution on $\mathbb{Z}^{d} \backslash\left\{x_{k}: k \leq i\right\}$. More precisely, for an event $B \subseteq\left(\mathbb{Z}^{d}\right)^{\mathbb{N}}$ on the space of possible paths for the walk,

$$
\mathbf{P}(B)=\mathrm{P}\left(B \times \Omega \mid \omega_{x_{1}}, \ldots, \omega_{x_{i}} ; A_{N}\right),
$$

and equivalently for $\mathbf{P}^{\prime}$. Then

$$
U_{i} \leq \sup _{\omega^{\prime}}\left|\mathbf{P}^{\prime}\left(X_{T_{M+V}}=v\right)-\mathbf{P}\left(X_{T_{M+V}}=v\right)\right|,
$$

where the supremum is taken over all environments $\omega^{\prime}$ that agree with $\omega$ on $\mathbb{Z}^{d} \backslash\left\{x_{i}\right\}$. Note that conditioned on the event that $x_{i}$ is not visited, the distributions $\mathbf{P}$ and $\mathbf{P}^{\prime}$ are the same. Now, for both measures $\mathbf{P}$ and $\mathbf{P}^{\prime}$, condition on the event that $x_{i}$ is visited. Let $u$ be the first regeneration point after $x_{i}$. Then $\mathbf{P}$ and $\mathbf{P}^{\prime}$ a.s, $\left\|u-x_{i}\right\|_{1}<d R(N)$. This follows from the conditioning on $A_{N}$. Therefore, from Lemma 4.2(3)\&(4) we get

$$
\begin{aligned}
\mid \mathbf{P}\left(X_{T_{M+V}}=v \mid x_{i} \text { is visited }\right)-\mathbb{P}^{x_{i}}\left(X_{T_{M+V}}=v\right) \mid<C R(N) V^{-d / 2}, \\
\mid \mathbf{P}^{\prime}\left(X_{T_{M+V}}=v \mid x_{i} \text { is visited }\right)-\mathbb{P}^{x_{i}}\left(X_{T_{M+V}}=v\right) \mid<C R(N) V^{-d / 2} .
\end{aligned}
$$

Hence

$$
U_{i} \leq C R(N) V^{-d / 2} \mathbf{P}\left(x_{i} \text { is visited }\right),
$$

and (4.30) follows. 
Using (4.30), conditioned on $J(N)$, and based on the same calculation as in (4.28) and (4.29), we get

$$
U=\operatorname{ess} \sup \left(\sum_{i=1}^{n} U_{i}^{2}\right) \leq R(N)^{6} V^{-d} .
$$

Therefore, by Lemma 4.8, for every $v \in H_{M+V}$ and every number $\delta$,

$$
\begin{aligned}
P\left(\mid E\left[P_{\omega}\left(X_{T_{M+V}}=v\right) ; A_{N} \mid \mathcal{G}\right]-\mathbb{P}\left(X_{T_{M+V}}=v ;\right.\right. & \left.\left.A_{N}\right) \mid>\delta\right) \\
& \leq 2 P\left(J(N)^{c}\right)+2 e^{-\frac{\delta^{2}}{2 R(N)^{6} V^{-d}}}
\end{aligned}
$$

In particular, if $\delta=\frac{1}{4} N^{1-d}=\frac{1}{4} V^{-d / 2} V^{\eta}$, with $\eta=\frac{d+(1-d) / \theta^{\prime}}{2}>0$, then we get

$$
P\left(\left|E\left[P_{\omega}\left(X_{T_{M+V}}=v ; A_{N}\right) \mid \mathcal{G}\right]-\mathbb{P}\left(X_{T_{M+V}}=v ; A_{N}\right)\right|>\frac{1}{4} N^{1-d}\right)=N^{-\xi(1)}
$$

and

$$
\begin{array}{r}
P\left(\left|E\left[P_{\omega}\left(X_{T_{M+V}}=v\right) \mid \mathcal{G}\right]-\mathbb{P}\left(X_{T_{M+V}}=v\right)\right|>\frac{1}{2} N^{1-d}\right) \leq P\left(\omega: P_{\omega}\left(A_{N}^{c}\right) \geq \frac{1}{4} N^{1-d}\right) \\
\quad+P\left(\left|E\left[P_{\omega}\left(X_{T_{M+V}}=v ; A_{N}\right) \mid \mathcal{G}\right]-\mathbb{P}\left(X_{T_{M+V}}=v ; A_{N}\right)\right|>\frac{1}{4} N^{1-d}\right)=N^{-\xi(1)} .
\end{array}
$$

Let $T(N)$ be the event that

$$
\left|E\left[P_{\omega}\left(X_{T_{M+V}}=v\right) \mid \mathcal{G}\right]-\mathbb{P}\left(X_{T_{M+V}}=v\right)\right| \leq \frac{1}{2} N^{1-d}
$$

for every $\frac{2}{5} N^{2} \leq M \leq N^{2}$ and every $v \in H_{M+V} \cap \mathcal{P}(0,2 N)$. Then $P(T(N))=1-$ $N^{-\xi(1)}$. Now consider $\omega \in T(N)$, and fix $\frac{2}{5} N^{2} \leq M \leq N^{2}$ and a cube $Q$ of side length $N^{\theta}$ which is contained in $H_{M}$. We want to estimate

$$
L(Q)=\left|P_{\omega}^{z}\left(X_{T_{M}} \in Q ; A_{N}\right)-\mathbb{P}^{z}\left(X_{T_{M}} \in Q ; A_{N}\right)\right| .
$$

Let $c(Q)$ be the center of the cube $Q$, and let $c^{\prime}(Q)=c(Q)+V \vartheta /\left\langle\vartheta, e_{1}\right\rangle$. Define

$$
\begin{aligned}
& Q^{(1)}=\left\{v \in H_{V+M}:\left\|v-c^{\prime}(Q)\right\|_{\infty}<\frac{1}{2}(0.9)^{1 / d} N^{\theta}\right\}, \\
& Q^{(2)}=\left\{v \in H_{V+M}:\left\|v-c^{\prime}(Q)\right\|_{\infty}<\frac{1}{2}(1.1)^{1 / d} N^{\theta}\right\} .
\end{aligned}
$$

Then by simple annealed estimates,

$$
\begin{aligned}
\mathbb{P}^{z}\left(X_{T_{V+M}} \in Q^{(1)}\right) & <\mathbb{P}^{z}\left(X_{T_{M}} \in Q\right)+N^{-\xi(1)}, \\
\mathbb{P}^{z}\left(X_{T_{V+M}} \in Q^{(2)}\right) & >\mathbb{P}^{z}\left(X_{T_{M}} \in Q\right)-N^{-\xi(1)}, \\
E\left[P_{\omega}^{z}\left(X_{T_{V+M}} \in Q^{(1)}\right) \mid \mathcal{G}\right] & <P_{\omega}^{z}\left(X_{T_{M}} \in Q\right)+N^{-\xi(1)}, \\
E\left[P_{\omega}^{z}\left(X_{T_{V+M}} \in Q^{(2)}\right) \mid \mathcal{G}\right] & >P_{\omega}^{z}\left(X_{T_{M}} \in Q\right)-N^{-\xi(1)} .
\end{aligned}
$$

From the definition of $T(N)$ and (4.33)-(4.36), it follows that $T(N) \subseteq B^{\theta}(N)$. Therefore, $P\left(B^{\theta}(N)\right) \geq P(T(N))=1-N^{-\xi(1)}$.

Using Lemma 4.12 as a building block, we can get a similar yet weaker result for every choice of $\theta$. 
Lemma 4.13. Assume the assumptions 1-3 from page 136. For every $0<\theta \leq 1$ and $h$ let $\bar{B}^{(\theta, h)}(N)$ be the event that for every $z \in \tilde{\mathcal{P}}(0, N)$, every $\frac{1}{2} N^{2} \leq M \leq N^{2}$ and every cube $Q$ of side length $N^{\theta}$ which is contained in $H_{M}$,

$$
P_{\omega}^{z}\left(X_{T_{M}} \in Q ; A_{N}\right) \leq R_{h}(N) N^{(\theta-1)(d-1)} .
$$

Then for every $0<\theta \leq 1$ there exists $h=h(\theta)$ such that $P\left(\bar{B}^{(\theta, h)}(N)\right)=1-N^{-\xi(1)}$.

Proof. We argue by descending induction on $\theta$. From Lemma 4.12, $P\left(\bar{B}^{(\theta, 1)}(N)\right)=$ $1-N^{-\xi(1)}$ for every $1 \geq \theta>(d-1) / d$. For the induction step, fix $\theta$ and assume that the statement of the lemma holds for some $\theta^{\prime}$ such that $\theta>\frac{d-1}{d} \theta^{\prime}$, and let $h^{\prime}=h\left(\theta^{\prime}\right)$. We write $\rho=\theta / \theta^{\prime}$. Let $\sigma$ be the natural shift of $\mathbb{Z}^{d}$. Let

$$
L=\bar{B}^{(\rho, 1)}(N) \cap \bigcap_{z \in \mathcal{P}(0,2 N)} \sigma_{z}\left(\bar{B}^{\left(\theta^{\prime}, h^{\prime}\right)}\left(\left[N^{\rho}\right]\right)\right) \cap T(N, \rho),
$$

where

$$
T(N, \rho)=\left\{\omega \in \Omega: \forall_{v \in \mathcal{P}(0, N)}, P_{\omega}^{v}\left(X_{T_{\partial \mathcal{P}\left(v,\left[N^{\rho}\right]\right)}} \notin \partial^{+} \mathcal{P}\left(v,\left[N^{\rho}\right]\right)\right)<e^{-R_{1}(N)}\right\} .
$$

Clearly, $P(L)=1-N^{-\xi(1)}$. Therefore, all we need to show is that for some $h$ and all $N$ large enough, we have $L \subseteq \bar{B}^{(\theta, h)}(N)$. To this end we fix $\omega \in L, z, \frac{1}{2} N^{2} \leq M \leq N^{2}$ and a cube $Q$ of side length $N^{\theta}$ in $\mathcal{P}(0, N) \cap H_{M}$. Let $x$ be the center of $Q$, and let $V=\left[N^{\rho}\right]^{2}$ and $x^{\prime}=x-V \vartheta /\left\langle\vartheta, e_{1}\right\rangle$.

Since

$$
\omega \in \bigcap_{z \in \mathcal{P}(0,2 N)} \sigma_{z}\left(\bar{B}^{\left(\theta^{\prime}, h^{\prime}\right)}\left(\left[N^{\rho}\right]\right)\right),
$$

we find that for every $v \in H_{M-V}$,

$$
P_{\omega}^{v}\left(X_{T_{M}} \in Q\right)<R_{h^{\prime}}(N) N^{\rho\left(\theta^{\prime}-1\right)(d-1)}=R_{h^{\prime}}(N) N^{(\theta-\rho)(d-1)} .
$$

We remember that by the Markov property and the fact that $\omega \in T(N, \rho)$,

$$
P_{\omega}^{z}\left(X_{T_{M}} \in Q\right)=\sum_{v \in H_{M-V} \cap \mathcal{P}\left(x^{\prime},\left[N^{\rho}\right]\right)} P_{\omega}^{z}\left(X_{T_{M-V}}=v\right) P_{\omega}^{v}\left(X_{T_{M}} \in Q\right)+N^{-\xi(1)} .
$$

Now, $H_{M-V} \cap \mathcal{P}\left(x^{\prime},\left[N^{\rho}\right]\right)$ is the union of $2^{d-1} R_{5}(N)^{d-1}<R_{6}(N)$ cubes of side length $N^{\rho}$.

Since $\omega \in \bar{B}^{(\rho, 1)}(N)$, for every cube $Q^{\prime}$ of side length $N^{\rho}$ that is contained in $H_{M-V} \cap$ $\mathcal{P}(0, N)$ we get

$$
P_{\omega}^{z}\left(X_{T_{M-V}} \in Q^{\prime}\right)<R_{1}(N) N^{(\rho-1)(d-1)} .
$$

Combining (4.38)-(4.40), we get

$$
\begin{aligned}
P_{\omega}^{z}\left(X_{T_{M}} \in Q\right) & \leq R_{6}(N) R_{h^{\prime}}(N) N^{(\theta-\rho)(d-1)} \cdot R_{1}(N) N^{(\rho-1)(d-1)}+N^{-\xi(1)} \\
& \leq R_{h}(N) N^{(\theta-1)(d-1)}
\end{aligned}
$$

for $h=\max \left(6, h^{\prime}\right)+1$.

Next we prove a lemma which significantly strengthens the previous one. For its proof we will use Lemma 4.13 and a more careful treatment of the proof technique of Lemma 4.12. We start with the following preliminary lemma: 
Lemma 4.14. Assume the assumptions 1-3 from page 136. Let $\mathcal{G}$ be the $\sigma$-algebra generated by $\left\{\omega(z):\left\langle z, e_{1}\right\rangle \leq N^{2}\right\}$. Let $\eta>0, V=\left[N^{\eta}\right]$ and let $B(N, V)$ be the event that for all $z \in \tilde{\mathcal{P}}(0, N)$ and all $v \in H_{N^{2}+V}$,

$$
\left|E\left[P_{\omega}^{z}\left(X_{T_{N^{2}+V}}=v\right) \mid \mathcal{G}\right]-\mathbb{P}^{z}\left[X_{T_{N^{2}+V}}=v\right]\right| \leq N^{1-d} V^{(1-d) / 6} .
$$

Then $P(B(N, V))=1-N^{-\xi(1)}$.

Proof. Let $v \in H_{N^{2}+V}$ and let $\theta>0$ be such that $\theta<\frac{1}{20} \eta$. Let $K$ be an integer such that $2^{-K} N^{2}>V \geq 2^{-K-1} N^{2}$, and for $1 \leq k<K$ define

$$
\mathcal{P}^{(k)}=\mathcal{P}(0, N) \cap\left\{x: 2^{-k-1} N^{2} \leq N^{2}-\left\langle x, e_{1}\right\rangle<2^{-k} N^{2}\right\} .
$$

In addition we take

$$
\begin{aligned}
\mathcal{P}^{(K)} & =\mathcal{P}(0, N) \cap\left\{x: 0 \leq N^{2}-\left\langle x, e_{1}\right\rangle<2^{-K} N^{2}\right\}, \\
\mathcal{P}^{(0)} & =\mathcal{P}(0, N) \cap\left\{x: N^{2} / 2 \leq N^{2}-\left\langle x, e_{1}\right\rangle\right\} .
\end{aligned}
$$

Moreover, we define

$$
F(v)=\left\{x \in \mathcal{P}(0, N):\|x-u(v, x)\| \leq\left|\left\langle v-x, e_{1}\right\rangle\right|^{1 / 2} R_{2}(N)\right\},
$$

where $u(v, x)$ is as in (3.3). Then, for $0 \leq k \leq K$, we define

$$
\mathcal{P}^{(k)}(v)=\mathcal{P}^{(k)} \cap F(v), \quad \hat{\mathcal{P}}^{(k)}(v)=\left\{y: \exists_{x \in \mathcal{P}^{(k)}(v)}\|x-y\|<R_{2}(N)\right\} .
$$

Note that $\mathcal{P}^{(k)}(v) \subseteq \hat{\mathcal{P}}^{(k)}(v)$

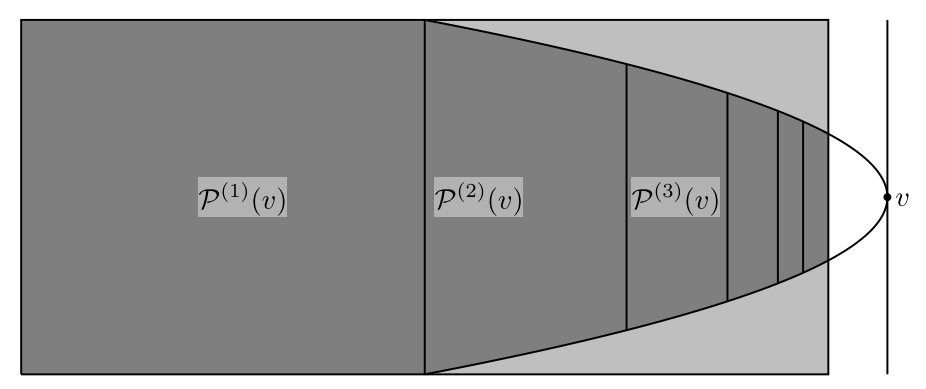

Fig. 4. The darker areas are $\mathcal{P}^{(k)}(v)$ for different values of $k$. The environment in the light-gray area has negligible influence on the probability of hitting $v$.

Condition on the event $\bar{B}^{(\theta, h)}$, with $h$ such that, by Lemma $4.13, P\left(\bar{B}^{(\theta, h)}\right)=$ $1-N^{\xi(1)}$.

For $0 \leq k \leq K$ and $\omega \in \bar{B}^{(\theta, h)}$, we want to estimate

$$
V(k)=E_{\omega, \omega}\left[\left[X^{(1)}\right] \cap\left[X^{(2)}\right] \cap \mathcal{P}^{(k)}(v)\right] .
$$

For $k=0$,

$$
V(0) \leq E_{\omega, \omega}\left[\left[X^{(1)}\right] \cap\left[X^{(2)}\right] \cap \mathcal{P}(0, N)\right] \leq R_{2}(N)
$$


For $k>0$,

$$
\begin{aligned}
V(k) & =\sum_{x \in \mathcal{P}^{(k)}(v)}\left[P_{\omega}^{z}(x \text { is visited })\right]^{2} \\
& \leq \sum_{x \in \mathcal{P}^{(k)}(v)}\left[\sum_{y:\|y-x\|<R(N)} P_{\omega}^{z}\left(X_{T_{\left\langle y, e_{1}\right\rangle}}=y\right)\right]^{2}+N^{-\xi(1)} \\
& \leq R(N)^{2 d} \sum_{y \in \hat{\mathcal{P}}^{(k)}(v)}\left[P_{\omega}^{z}\left(X_{T_{\left\langle y, e_{1}\right\rangle}}=y\right)\right]^{2}+N^{-\xi(1)} \\
& \leq R_{2}(N) \sum_{y \in \hat{\mathcal{P}}^{(k)}(v)} R_{h}(N) N^{2(1-\theta)(1-d)} \\
& \leq R_{h+1}(N) N^{2((d+1) / 2+(1-\theta)(1-d))} 2^{-k[(d+1) / 2]}
\end{aligned}
$$

where the inequality (4.41) follows from the fact that $\omega \in \bar{B}^{(\theta, h)}(N)$.

As before, we now use the same filtration $\left\{\mathcal{F}_{i}\right\}$ as in the proof of Lemma 4.11, and consider the martingale $M_{i}=E\left[P_{\omega}^{z}\left(X_{T_{N^{2}+V}}=v ; A_{N}\right) \mid \mathcal{F}_{i}\right]$. Again, in order to use Lemma 4.8, we need to bound $U_{i}=\operatorname{ess} \sup \left(\left|M_{i}-M_{i-1}\right| \mid \mathcal{F}_{i-1}\right)$. Let $x$ be such that $\omega_{x}$ is measurable with respect to $\mathcal{F}_{i}$ but not with respect to $\mathcal{F}_{i-1}$. Then $U_{i}=N^{-\xi(1)}$ if $x \notin F(v)$, while if $x \in F(v)$, then

$$
U_{i} \leq R(n) E\left[P_{\omega}^{z}(x \text { is hit }) \mid \mathcal{F}_{i-1}\right] D\left(N^{2}+V-\left\langle x, e_{1}\right\rangle\right)
$$

where $D(n)$ is the maximal first derivative of the annealed distribution at distance $n$. By Lemma 4.2, $D\left(N^{2}+V-\left\langle x, e_{1}\right\rangle\right) \leq C N^{-d} 2^{k d / 2}$ for $x \in \mathcal{P}^{(k)}(v)$. Therefore,

$$
\begin{aligned}
U & =\operatorname{ess} \sup \left(\sum_{i} U_{i}^{2}\right) \leq C \sum_{k=0}^{K} V(K) N^{-2 d} 2^{k d}+N^{-\xi(1)} \\
& \leq C R_{h+1}(N) N^{-2 d}+C R_{h+1}(N) N^{2((d+1) / 2+(1-\theta)(1-d))-2 d} \sum_{k=1}^{K} 2^{k d-k(d+1) / 2}+N^{-\xi(1)} \\
& \leq C R_{h+1}(N)\left(N^{-2 d}+N^{3-3 d+2(d-1) \theta} 2^{K(d-1) / 2}\right) \\
& \leq C R_{h+1}(N)\left(N^{-2 d}+N^{2-2 d+2(d-1) \theta} V^{-(d-1) / 2}\right) \leq C N^{2-2 d} V^{-(d-1) / 6+\epsilon}
\end{aligned}
$$

for small enough $\epsilon$.

Therefore, using Lemma 4.8, with probability $1-N^{-\xi(1)}$,

$$
\left|E\left[P_{\omega}^{z}\left(X_{T_{N^{2}+V}}=v ; A_{N}\right) \mid \mathcal{G}\right]-\mathbb{P}\left[X_{T_{N^{2}+V}}=v ; A_{N}\right]\right| \leq N^{1-d} V^{(1-d) / 6} .
$$

A simple union bound coupled with the fact that $\mathbb{P}\left(A_{N}\right)=N^{-\xi(1)}$ completes the proof of the lemma.

Lemma 4.15. Assume the assumptions 1-3 from page 136. For every $0<\theta \leq 1$ let $D^{(\theta)}(N) \subseteq \Omega$ be the event that for every $z \in \tilde{\mathcal{P}}(0, N)$ and every cube $Q$ of side length $N^{\theta}$ which is contained in $\partial^{+} \mathcal{P}(0, N)$,

$$
\left|P_{\omega}^{z}\left(X_{T_{\partial \mathcal{P}(0, N)}} \in Q\right)-\mathbb{P}^{z}\left(X_{T_{\partial \mathcal{P}(0, N)}} \in Q\right)\right| \leq N^{(\theta-1)(d-1)-\theta \frac{d-1}{d+1}} .
$$

Then $P\left(D^{(\theta)}(N)\right)=1-N^{-\xi(1)}$. 
Proof. Take $\frac{3}{4} \theta<\theta^{\prime}<\theta$ and $V=\left[N^{8 \theta^{\prime} /(d+1)}\right]$. Then by Lemma 4.14 we know that $P(B(N, V))=1-N^{-\xi(1)}$. As before, all we need to show is that $B(N, V) \subseteq D^{(\theta)}(N)$. The way we do this will be completely identical to the last step of the proof of Lemma 4.12. Let $\omega \in B(N, V)$, and let $Q$ be a cube of side length $N^{\theta}$ which is contained in $\partial^{+} \mathcal{P}(0, N)$. Let $x$ be the center of $Q$, and let $x^{\prime}=x+V \vartheta /\left\langle\vartheta, e_{1}\right\rangle$.

Let $Q^{(1)}$ and $Q^{(2)}$ be $d-1$-dimensional cubes that are contained in $H_{N^{2}+V}$ and centered at $x^{\prime}$, of side length $N^{\theta}-R_{3}(N) \sqrt{V}$ and $N^{\theta}+R_{3}(N) \sqrt{V}$ respectively. Then, on $B(N, V)$, for $i=1,2$,

$$
\left|E\left[P_{\omega}^{z}\left(X_{T_{N^{2}+V}} \in Q^{(i)}\right) \mid \mathcal{G}\right]-\mathbb{P}\left[X_{T_{N^{2}+V}} \in Q^{(i)}\right]\right| \leq\left|Q^{(i)}\right| N^{1-d} V^{(1-d) / 6} .
$$

In addition, exactly as in the proof of Lemma 4.12,

$$
\begin{aligned}
\mathbb{P}^{z}\left(X_{T_{V+N^{2}}} \in Q^{(1)}\right) & <\mathbb{P}^{z}\left(X_{T_{N^{2}}} \in Q\right)+N^{-\xi(1)}, \\
\mathbb{P}^{z}\left(X_{T_{V+N^{2}}} \in Q^{(2)}\right) & >\mathbb{P}^{z}\left(X_{T_{N^{2}}} \in Q\right)-N^{-\xi(1)}, \\
E\left[P_{\omega}^{z}\left(X_{T_{V+N^{2}}} \in Q^{(1)}\right) \mid \mathcal{G}\right] & <P_{\omega}^{z}\left(X_{T_{N^{2}}} \in Q\right)+N^{-\xi(1)}, \\
E\left[P_{\omega}^{z}\left(X_{T_{V+N^{2}}} \in Q^{(2)}\right) \mid \mathcal{G}\right] & >P_{\omega}^{z}\left(X_{T_{N^{2}}} \in Q\right)-N^{-\xi(1)} .
\end{aligned}
$$

Therefore, for $\omega \in B(N, V)$,

$$
\begin{aligned}
& \left|P_{\omega}^{z}\left(X_{T_{\partial \mathcal{P}(0, N)}} \in Q\right)-\mathbb{P}^{z}\left(X_{T_{\partial \mathcal{P}(0, N)}} \in Q\right)\right| \\
& \quad \leq\left(\left|Q^{(1)}\right|+\left|Q^{(2)}\right|\right) N^{1-d} V^{(1-d) / 6}+C\left(\left|Q^{(2)}\right|-\left|Q^{(1)}\right|\right) N^{1-d}+N^{-\xi(1)} \\
& \quad \leq C\left(N^{(1-\theta)(1-d)} V^{(1-d) / 6}+R_{3}(N) N^{(1-d)+(d-2) \theta} \sqrt{V}\right) .
\end{aligned}
$$

The lemma follows from the choice of $V$.

Proof of Proposition 4.5. The proposition follows from Lemmas 4.11 and 4.15.

\subsection{Sums of approximate gaussians}

The purpose of this subsection is to prove Lemma 4.16 below. Let $\mathcal{D}(N)$ be the annealed distribution starting from zero of $X_{T_{\partial \mathcal{P}(0, N)}}$ conditioned on $\partial^{+} \mathcal{P}(0, N)$.

Lemma 4.16. Assume the assumptions 1-3 from page 136. Let $0<\lambda<1$ and $n$ be so that $n<\lambda^{-1}$. Let $K$ be so that $N>K \geq 1$. Let $h \geq 5$. Assume further that $N>K^{4}$ and $N>\lambda^{-4}$, and that $\lambda N>2 K n R_{h+1}(N)$. Let $\left\{X_{i}\right\}_{i=1}^{n}$ be random variables such that for every $i$, conditioned on $X_{1}, \ldots, X_{i-1}$, the distribution of $X_{i}$ is $(\lambda, K)$-close to $\mathcal{D}(N)$. Let $S=\sum_{i=1}^{n} X_{i}$. Then the distribution of $S$ is $\left(\lambda R_{h+1}(N), 2 n K R_{h+1}(N)\right)$-close to $\mathcal{D}(N \sqrt{n})$.

Remark. We need the assumptions 1-3 because they give us some control over the distribution $\mathcal{D}(N)$.

We use the following simple fact, which follows from the decomposition of the annealed RWRE into regenerations. 
Claim 4.17. Assume the assumptions $1-3$ from page 136. For $j>1$, let $\hat{\mathcal{D}}^{(j)}$ be the convolution of $\mathcal{D}(N)$ and $\mathcal{D}(N \sqrt{j-1})$. Let $U \sim \hat{\mathcal{D}}^{(j)}$. Then $U$ can be represented as $U=\hat{U}+U^{\prime}$ with $\hat{U} \sim \mathcal{D}(N \sqrt{j})$ and for every $k$,

$$
\mathbf{P}\left(\left\|U^{\prime}\right\|>k\right)<C e^{-c k^{\gamma}}+N^{-\xi(1)}
$$

for some constants $C$ and $c$. In particular, there exists some constant $C$, independent of $N$ and $j$, such that

$$
\left\|E\left(U^{\prime}\right)\right\| \leq E\left(\left\|U^{\prime}\right\|\right)<C .
$$

Proof. (4.49) follows immediately from (4.48) (in order to handle the $N^{-\xi(1)}$ error, note that $U^{\prime}$ is bounded by $3 N R_{5}(N)$ ), and therefore we shall only prove (4.48).

We will define a coupling between a random variable $U$ which is approximately $\hat{\mathcal{D}}^{(j)}$ distributed and a random variable $\hat{U}$ which is approximately $\mathcal{D}(N \sqrt{j})$ distributed such that

$$
\mathbf{P}(\|U-\hat{U}\|>k)<C e^{-c k^{\gamma}}+N^{-\xi(1)} .
$$

To construct the coupling, we define an ensemble $\mathbb{L}=\{\mathbb{U}, \mathbb{T}\}$ where $\mathbb{U}$ is a positive integer, and $\mathbb{T}$ is a nearest neighbor path of length $\mathbb{U}$, taking values in $\mathbb{Z}^{d}$ and starting at 0 .

Let $\left\{\mathbb{L}_{n}=\left\{\mathbb{U}_{n}, \mathbb{T}_{n}\right\}\right\}_{n=1}^{\infty}$ be i.i.d. ensembles such that $\mathbb{U}_{1}$ is sampled according to the annealed distribution of $\tau_{2}-\tau_{1}$, and the path $\mathbb{T}_{1}$ is distributed according to the annealed distribution of $X_{\tau_{1}+\cdot}-X_{\tau_{1}}$, run up to time $\tau_{2}-\tau_{1}$ and conditioned on $\tau_{2}-\tau_{1}=\mathbb{U}_{1}$.

Additionally, define $\hat{\mathbb{L}}_{1}=\left\{\hat{\mathbb{U}}_{1}, \hat{\mathbb{T}}_{1}\right\}$ and $\hat{\mathbb{L}}_{2}=\left\{\hat{\mathbb{U}}_{2}, \hat{\mathbb{T}}_{2}\right\}$ to be two i.i.d. ensembles such that $\hat{\mathbb{U}}_{1}$ is sampled according to the annealed distribution of $\tau_{1}$ and $\hat{\mathbb{T}}_{1}$ is distributed according to the annealed distribution of $X$., run up to time $\tau_{1}$ and conditioned on $\tau_{1}=\mathbb{U}_{1}$. In addition, we require independence of $\hat{\mathbb{L}}_{1}$ and $\hat{\mathbb{L}}_{2}$ and $\left\{\mathbb{L}_{n}\right\}_{n=1}^{\infty}$.

In other words, $\hat{\mathbb{L}}_{1}$ and $\hat{\mathbb{L}}_{2}$ are distributed according to the annealed distribution of the first regeneration slab, and $\left\{\mathbb{L}_{n}\right\}$ are distributed according to the annealed distribution of regeneration slabs that are not the first one.

We now construct paths from the ensembles we defined. The choice of the distribution of the ensembles will guarantee that the paths are distributed according to the annealed RWRE distribution. The variables $U$ and $\hat{U}$ will be taken to be certain hitting locations of these paths, and the fact that $U$ and $\hat{U}$ will be built from the same ensembles will make it easy for us to estimate the difference $U-\hat{U}$.

Let $\Gamma_{n}=\hat{\mathbb{T}}_{1}\left(\hat{\mathbb{U}}_{1}\right)+\sum_{k=1}^{n} \mathbb{T}_{k}\left(\mathbb{U}_{k}\right)$, and let $T_{1}=\max \left(h:\left\langle e_{1}, \Gamma_{h}\right\rangle<N^{2} j\right)$. We take

$$
\hat{U}=\Gamma_{T_{1}}+\mathbb{T}_{T_{1}+1}\left(\min \left(i:\left\langle e_{1}, \mathbb{T}_{T_{1}+1}(i)+\Gamma_{T_{1}}\right\rangle=N^{2} j\right)\right) .
$$

Let $T_{2}=\max \left(h:\left\langle e_{1}, \Gamma_{h}\right\rangle<N^{2}(j-1)\right)$, and

$$
V_{1}=\Gamma_{T_{2}}+\mathbb{T}_{T_{2}+1}\left(\min \left(i:\left\langle e_{1}, \mathbb{T}_{T_{2}+1}(i)+\Gamma_{T_{2}}\right\rangle=N^{2}(j-1)\right)\right) .
$$

Let $\Gamma_{n}^{\prime}=\hat{\mathbb{T}}_{2}\left(\hat{\mathbb{U}}_{2}\right)+\Gamma_{T_{2}+n}-\Gamma_{T_{2}}$. Let $T_{3}=\max \left(h:\left\langle e_{1}, \Gamma_{h}^{\prime}\right\rangle<N^{2}\right)$, and

$$
V_{2}=\Gamma_{T_{3}}^{\prime}+\mathbb{T}_{T_{2}+T_{3}+1}\left(\min \left(i:\left\langle e_{1}, \mathbb{T}_{T_{2}+T_{3}+1}(i)+\Gamma_{T_{2}+T_{3}}\right\rangle=N^{2}\right)\right) .
$$

We now take $U=V_{1}+V_{2}$. 
By Lemmas 4.1 and 4.2(1), up to an error of $N^{-\xi(1)}$, the variables $U$ and $\hat{U}$ are distributed (respectively) according to $\hat{\mathcal{D}}^{(j)}$ and $\mathcal{D}(N \sqrt{j})$.

The difference $U-\hat{U}$ is bounded by the sums of the radii of the regeneration slabs $\hat{\mathbb{L}}_{2}, \mathbb{L}_{T_{2}}$, and $\mathbb{L}_{h}$ for $h$ between $T_{2}+T_{3}$ and $T_{1}$. Lemma 4.1 now gives us the desired bound.

We also use the following lemma, which is nothing but a second order Taylor expansion.

Lemma 4.18. Let $\mu$ be a finite signed measure on $\mathbb{Z}^{d}$, and let $f: \mathbb{Z}^{d} \rightarrow \mathbb{R}$. Assume that $m, k, J, L$ in $\mathbb{N}$ and $\varrho \in \mathbb{Z}^{d}$ are such that:

(1) For every $x$, $y$ such that $x-y \in\left\{ \pm e_{i}\right\}_{i=1}^{d}$, we have $|f(x)-f(y)|<m$.

(2) For every $x, y, z, w$ and $1 \leq i, j \leq d$ such that $x-y=z-w=e_{i}$ and $x-z=$ $y-w=e_{j}$, we have $|f(x)+f(w)-f(y)-f(z)|<k$ (note that if $i=j$ then this is the discrete pure second derivative, and if $i \neq j$ it is the discrete mixed second derivative).

(3) $\sum_{x} \mu(x)=0$.

(4) $\left\|\sum_{x} x \mu(x)\right\|_{1}<L$.

(5) $\sum_{x}\|x-\varrho\|_{1}^{2}|\mu(x)|<J$.

Then

$$
\left|\sum_{x} \mu(x) f(x)\right| \leq L m+\frac{1}{2} J k .
$$

Proof. Since $\sum_{x} \mu(x)=0$ we have $\sum_{x} \mu(x) f(x)=\sum_{x} \mu(x)(f(x)+c)$ for every $c$. Therefore, without loss of generality we may assume that $f(\varrho)=0$. Let $g: \mathbb{R}^{d} \rightarrow \mathbb{R}$ be the affine function such that $g(\varrho)=f(\varrho)=0$ and $g\left(\varrho+e_{i}\right)=f\left(\varrho+e_{i}\right)$ for $i=1, \ldots, d$. Then $|f(x)-g(x)|<\frac{1}{2} k\|x-\varrho\|_{1}^{2}$ for $x \in \mathbb{Z}^{d}$. Note also that since $\sum_{x} \mu(x)=0$, we get $\sum_{x}(x-\varrho) \mu(x)=\sum_{x} x \mu(x)$ and thus $\left\|\sum_{x}(x-\varrho) \mu(x)\right\|<L$. Therefore,

$$
\left|\sum_{x} \mu(x) f(x)-\sum_{x} \mu(x) g(x)\right| \leq \sum_{x}|\mu(x)||f(x)-g(x)| \leq \frac{1}{2} J k .
$$

In addition,

$$
\left|\sum_{x} \mu(x) g(x)\right|=\left|g\left(\sum_{x}(x-\varrho) \mu(x)\right)\right| \leq L m .
$$

The lemma follows.

Proof of Lemma 4.16. For $k=1, \ldots, n$, conditioned on $X_{1}, \ldots, X_{k-1}$, the distribution of $X_{k}$ is $(\lambda, K)$-close to $\mathcal{D}(N)$. Therefore there exist variables $\left\{Y_{k}\right\}_{k=1}^{n}$, playing the role of $Z_{0}$ in Definition 11, such that for every $k$, conditioned on $X_{1}, Y_{1}, \ldots, X_{k-1}, Y_{k-1}$, the following hold:

- $\sum_{x}\left|\mathbf{P}\left(Y_{k}=x\right)-\mathcal{D}(N)(x)\right| \leq \lambda$.

- $\mathbf{P}\left(\left\|Y_{k}-X_{k}\right\|<K\right)=1$.

- $E\left(Y_{k}\right)=E_{\mathcal{D}(N)}$.

- $\sum_{x}\left|\mathbf{P}\left(Y_{k}=x\right)-\mathcal{D}(N)(x)\right|\left\|x-E_{\mathcal{D}(N)}\right\|_{1}^{2} \leq \lambda N^{2}$. 
What we need to show is that there exists a random variable $Y^{\prime}$ such that:

(1) $\sum_{x}\left|\mathbf{P}\left(Y^{\prime}=x\right)-\mathcal{D}(\sqrt{n} N)(x)\right| \leq \lambda R_{h+1}(N)$.

(2) $\mathbf{P}\left(\left\|Y^{\prime}-S\right\|<2 n K R_{h+1}(N)\right)=1$.

(3) $E\left(Y^{\prime}\right)=E_{\mathcal{D}(\sqrt{n} N)}$.

(4) $\sum_{x}\left|\mathbf{P}\left(Y^{\prime}=x\right)-\mathcal{D}(\sqrt{n} N)(x)\right|\left\|x-E_{\mathcal{D}(\sqrt{n} N)}\right\|_{1}^{2} \leq \lambda n N^{2} R_{h+1}(N)$.

To this end, we let

$$
S^{(j)}=\sum_{k=j}^{n} Y_{k}
$$

First we will show, using descending induction, that conditioned on $X_{1}, \ldots, X_{j-1}$, we can represent $S^{(j)}$ as $S^{(j)}=Y^{(j)}+Z^{(j)}$ such that $\left\|Z^{(j)}\right\| \leq(n-j) R_{h}(N)$ a.s. and $Y^{(j)} \sim \mathcal{D}(N \sqrt{n-j+1})+D_{2}^{(j)}$ where $D_{2}^{(j)}$ is a signed measure such that $\left\|D_{2}^{(j)}\right\| \leq \lambda^{(j)}$ with $\lambda^{(n)}=\lambda$ and $\lambda^{(j)} \leq \lambda^{(j+1)}+\frac{2}{n-j} \lambda R_{5}(N)$ for $j<n$.

For $j=n$ the statement clearly holds, with $Z^{(n)}=0$. We now assume that the statement holds for $j+1$, and prove it for $j$.

Let $\mathbf{P}$ be the joint distribution of $Y_{j}$ and $Y^{(j+1)}$ conditioned on $X_{1}, \ldots, X_{j-1}$. Let $H=Y_{j}+Y^{(j+1)}$. For each $z$,

$$
\mathbf{P}(H=z)=\sum_{x} \mathbf{P}\left(Y_{j}=x\right) \mathbf{P}\left(Y^{(j+1)}=z-x \mid Y_{j}=x\right)
$$

Let $\mathcal{D}^{(j)}$ be the convolution of $\mathcal{D}(N \sqrt{n-j})$ and the $\mathbf{P}$ distribution of $Y_{j}$. Then

$$
\begin{aligned}
& \sum_{z}\left|\mathbf{P}(H=z)-\mathcal{D}^{(j)}(z)\right| \\
& \leq \sum_{z} \sum_{x} \mathbf{P}\left(Y_{j}=x\right)\left|\mathbf{P}\left(Y^{(j+1)}=z-x \mid Y_{j}=x\right)-\mathcal{D}(N \sqrt{n-j})(z-x)\right| \\
& =\sum_{x, y} \mathbf{P}\left(Y_{j}=x\right)\left|\mathbf{P}\left(Y^{(j+1)}=y \mid Y_{j}=x\right)-\mathcal{D}(N \sqrt{n-j})(y)\right| \\
& \leq \operatorname{ess} \sup \left\|D_{2}^{(j+1)}\right\| .
\end{aligned}
$$

As in Claim 4.17 let $\hat{\mathcal{D}}^{(j)}$ be the convolution of $\mathcal{D}(N)$ and $\mathcal{D}(N \sqrt{n-j})$. Then for given $z$, by Lemmas 4.18 and $4.2(5) \&(6)$,

$$
\begin{aligned}
\left|\hat{\mathcal{D}}^{(j)}(z)-\mathcal{D}^{(j)}(z)\right| & =\sum_{x} \mathcal{D}(N \sqrt{n-j})(x)\left(\mathbf{P}\left(Y_{j}=z-x\right)-\mathcal{D}(N)(z-x)\right) \\
& \leq \lambda N^{2} \cdot N^{-d-1}(n-j)^{(-d-1) / 2}=\lambda N^{1-d}(n-j)^{(-d-1) / 2} .
\end{aligned}
$$

Note that for $z$ such that $\left\|z-E_{\hat{\mathcal{D}}^{(j)}}\right\|_{1}>R_{5}(N) N(n-j)^{1 / 2}$, both $\hat{\mathcal{D}}^{(j)}(z)$ and $\mathcal{D}^{(j)}(z)$ are bounded by

$$
\exp \left(-\left(\frac{\left\|z-E_{\hat{\mathcal{D}}^{(j)}}\right\|_{1}}{R_{1}(N)}\right)^{2} / N^{2(d-1)}(n-j)^{d-1}\right) \leq e^{-R_{4}(N)} .
$$


From (4.50)-(4.52), we deduce that the distribution of $H$ can be represented as $\hat{\mathcal{D}}^{(j)}+$ $\bar{D}_{2}^{(j)}$ with

$$
\left\|\bar{D}_{2}^{(j)}\right\| \leq\left\|D_{2}^{(j+1)}\right\|+\lambda(N) R_{5}(N)(n-j)^{-1} .
$$

By Claim 4.17, and again conditioned on $X_{1}, Y_{1}, \ldots, X_{j-1}, Y_{j-1}$, there exists $Z^{\prime}(j)$ such that $\left.\mathbf{P}\left(Z^{\prime}(j)\right)>R_{h}(N)\right)<\exp \left(-R_{h-1}(N)\right)$, and the distribution of $H+Z^{\prime}(j)$ is $\mathcal{D}(N \sqrt{n-j+1})^{(j)}+\bar{D}_{2}^{(j)}$.

Let

$$
\bar{H}(j)=H+Z^{\prime}(j) \cdot \mathbf{1}_{\left\|Z^{\prime}(j)\right\|<R_{h}(N)} .
$$

Then the distribution of $\bar{H}(j)$ is $\mathcal{D}(N \sqrt{n-j+1})^{(j)}+\hat{D}_{2}^{(j)}$ with

$$
\left\|\hat{D}_{2}^{(j)}\right\| \leq\left\|\bar{D}_{2}^{(j)}\right\|+\exp \left(-R_{h-1}(N)\right) \leq\left\|D_{2}^{(j+1)}\right\|+2 \lambda(N) R_{5}(N)(n-j)^{-1} .
$$

We let

$$
Z^{(j)}=Z^{(j+1)}+Z^{\prime}(j) \cdot \mathbf{1}_{\left\|Z^{\prime}(j)\right\|<R_{h}(N)}
$$

and $Y^{(j)}=S^{(j)}-Z^{(j)}$. Then $\left\|Z^{(j)}\right\| \leq(n-j) R_{h}(N)$ and the distribution of $Y^{(j)}$ is $\left.\mathcal{D}(N \sqrt{n-j+1})+D_{2}^{(j)}\right)$ where $D_{2}^{(j)}$ is a signed measure such that $\left\|D_{2}^{(j)}\right\| \leq \lambda^{(j)}$ with

$$
\lambda^{(j)} \leq \lambda^{(j+1)}+\frac{2 R_{5}(N)}{n-j} \lambda .
$$

We calculate the expectation of $Y^{(1)}$ :

$$
E\left(Y^{(1)}\right)=E\left(S^{(1)}\right)-E\left(Z^{(1)}\right)=n E\left(Y_{1}\right)-E\left(Z^{(1)}\right)=n E_{\mathcal{D}(N)}-E\left(Z^{(1)}\right) .
$$

Therefore, again by Claim 4.17,

$$
\left\|E\left(Y^{(1)}\right)-E_{\mathcal{D}(\sqrt{n} N)}\right\| \leq C n+n R_{h}(N)<n R_{h+1}(N) .
$$

As in the proof of Corollary 4.6, we can find a variable $U$ which is independent of all of the variables we have seen so far, such that $\|U\| \leq n R_{h+1}(N)+1$ almost surely and $E(U)=E_{\mathcal{D}(\sqrt{n} N)}-E\left(Y^{(1)}\right)$.

We define $Y^{\prime}=Y^{(1)}+U$. By the same calculation as in (4.20), we find that $Y^{\prime}$ satisfies (1)-(3).

Thus, all that is left is to show that $Y^{\prime}$ also satisfies (4). To this end, let $D_{2}$ be the signed measure such that $Y^{\prime} \sim \mathcal{D}(\sqrt{n} N)+D_{2}$. We are interested in

$$
\sum_{x}\left|D_{2}(x)\right|\left\|x-E_{\mathcal{D}(\sqrt{n} N)}\right\|_{1}^{2} .
$$

As a first step, we estimate

$$
\operatorname{var}\left(D_{2}, i\right):=\sum_{z}\left\langle z, e_{i}\right\rangle^{2} D_{2}(z)
$$

for a unit vector $e_{i}$ with $i \neq 1$.

For $x, y, z \in \mathbb{Z}^{d}$, we write $\hat{x}, \hat{y}, \hat{z}$ for their projections on the $e_{i}$ axis.

Let $W$ be a random variable distributed according to $\mathcal{D}(\sqrt{n} N)$. By Claim 4.17, there exists another random variable $W^{\prime}$ such that $W^{\prime} \sim \mathcal{D}(N)^{\star n}$ (the $n$-fold convolution of $\mathcal{D}(N))$ and $\mathbf{P}\left(\left\|W-W^{\prime}\right\|>n k\right)<C n \exp \left(-c k^{-\gamma}\right)$ for every $k$. 
By the definition of $Y^{\prime}$, we know that $U^{\prime}=Y^{\prime}-S^{(1)}$ satisfies $\left\|U^{\prime}\right\| \leq 2 n R_{h+1}(N)$.

In addition note that $\operatorname{cov}\left(Y_{j}, Y_{k}\right)=0$ for $j \neq k$, and for every $j$,

$$
\begin{aligned}
\left|\operatorname{var}\left(\left\langle Y_{j}, e_{i}\right\rangle\right)-\operatorname{var}_{\mathcal{D}(N)}(\hat{x})\right| & =\sum_{x}\left(\hat{x}-E_{\mathcal{D}(N)}(\hat{z})\right)^{2}\left(\mathbf{P}\left(Y_{j}=x\right)-\mathcal{D}(N)(x)\right) \\
& \leq \sum_{x}\left(\hat{x}-E_{\mathcal{D}(N)}(\hat{z})\right)^{2}\left|\mathbf{P}\left(Y_{j}=x\right)-\mathcal{D}(N)(x)\right| \leq \lambda N^{2} .
\end{aligned}
$$

Therefore,

$$
\begin{array}{r}
\left|\operatorname{var}\left(\left\langle S^{(1)}, e_{i}\right\rangle\right)-\operatorname{var}\left(\left\langle W^{\prime}, e_{i}\right\rangle\right)\right|=\left|E\left(\left\langle S^{(1)}, e_{i}\right\rangle^{2}\right)-E\left(\left\langle W^{\prime}, e_{i}\right\rangle^{2}\right)\right| \\
\leq \sum_{j=1}^{n}\left|\operatorname{var}\left(\left\langle Y_{j}, e_{i}\right\rangle\right)-\operatorname{var}_{\mathcal{D}(N)}(\hat{x})\right| \leq \lambda n N^{2} .
\end{array}
$$

Now,

$$
\begin{aligned}
\mid \operatorname{var}\left(\left\langle Y^{\prime}, e_{i}\right\rangle\right) & -\operatorname{var}\left(\left\langle S^{(1)}, e_{i}\right\rangle\right)|=| \operatorname{var}\left\langle S^{(1)}+U^{\prime}, e_{i}\right\rangle-\operatorname{var}\left\langle S^{(1)}, e_{i}\right\rangle \mid \\
& \leq 2 \operatorname{ess} \sup \left(\left\|U^{\prime}\right\|\right) \sqrt{\operatorname{var}\left(S^{(1)}\right)}+\operatorname{ess} \sup \left(\left\|U^{\prime}\right\|\right)^{2} \\
& \leq 2 C n^{3 / 2} R_{h+1}(N) N+n^{2} R_{h+1}(N)^{2} \leq 3 C n^{3 / 2} R_{h+1}(N) N,
\end{aligned}
$$

and

$$
\begin{aligned}
& \left|\operatorname{var}\left(\left\langle W, e_{i}\right\rangle\right)-\operatorname{var}\left(\left\langle W^{\prime}, e_{i}\right\rangle\right)\right| \\
& \quad \leq N^{2} n^{2} \mathbf{P}\left(\left\|W-W^{\prime}\right\|>n R_{5}(N)\right)+2 n R_{5}(N) \sqrt{\operatorname{var}\left(W^{\prime}\right)}+2 n^{2} R_{5}(N)^{2} \\
& \quad \leq C n^{3 / 2} R_{5}(N) N .
\end{aligned}
$$

From (4.53)-(4.55) and the fact that $E\left(Y^{\prime}\right)=E(W)$, we get

$$
\begin{aligned}
\left|\operatorname{var}\left(D_{2}, i\right)\right| & =\left|\sum_{x}\left\langle x, e_{i}\right\rangle^{2}\left(\mathcal{D}(\sqrt{n} N)(x)-\mathbf{P}\left(Y^{\prime}=x\right)\right)\right| \\
& =\left|E\left(\left\langle W, e_{i}\right\rangle^{2}\right)-E\left(\left\langle Y^{\prime}, e_{i}\right\rangle^{2}\right)\right|=\left|\operatorname{var}\left(\left\langle W, e_{i}\right\rangle\right)-\operatorname{var}\left(\left\langle Y^{\prime}, e_{i}\right\rangle\right)\right| \\
& \leq \lambda n N^{2}+4 C n^{3 / 2} R_{h}(N) N \leq 2 \lambda n N^{2} .
\end{aligned}
$$

We now decompose the measure $D_{2}$ into its positive and negative parts, $D_{2}^{+}$and $D_{2}^{-}$. We need to bound

$$
\sum_{x}\left(\hat{x}-E_{\mathcal{D}(\sqrt{n} N)}\right)^{2}\left|D_{2}\right|=\sum_{x}\left(\hat{x}-E_{\mathcal{D}(\sqrt{n} N)}\right)^{2} D_{2}^{+}+\sum_{x}\left(\hat{x}-E_{\mathcal{D}(\sqrt{n} N)}\right)^{2} D_{2}^{-} .
$$

We know that

$$
\begin{aligned}
& \left|\sum_{x}\left(\hat{x}-E_{\mathcal{D}(\sqrt{n} N)}\right)^{2} D_{2}^{+}(x)-\sum_{x}\left(\hat{x}-E_{\mathcal{D}(\sqrt{n} N)}\right)^{2} D_{2}^{-}(x)\right| \\
& =\left|\sum_{x} \hat{x}^{2} D_{2}^{+}(x)-\sum_{x} \hat{x}^{2} D_{2}^{-}(x)\right|=\left|\operatorname{var}\left(D_{2}, i\right)\right| \leq 2 \lambda n N^{2} .
\end{aligned}
$$

In addition, note that $D_{2}^{-}(x) \leq \mathcal{D}(\sqrt{n} N)(x)$ for all $x$, and therefore

$$
D_{2, n}^{-}(x)<e^{-\left(x-E_{\mathcal{D}(\sqrt{n} N)}\right)^{2} / C n N^{2} R_{1}(N)} .
$$


Combining this with the fact that $\left\|D_{2}^{-}\right\| \leq\left\|D_{2}\right\| \leq \lambda R_{h}(N)$, we get

$$
\sum_{x}\left(\hat{x}-E_{\mathcal{D}(\sqrt{n} N)}\right)^{2} D_{2}^{-} \leq R_{2}(N) R_{h}(N) \lambda n N^{2} .
$$

Thus, by (4.57) and (4.58),

$$
\begin{aligned}
& \sum_{x}\left(\hat{x}-E_{\mathcal{D}(\sqrt{n} N)}\right)^{2} D_{2}^{+}+\sum_{x}\left(\hat{x}-E_{\mathcal{D}(\sqrt{n} N)}\right)^{2} D_{2}^{-} \\
& \quad \leq 2 \sum_{x}\left(\hat{x}-E_{\mathcal{D}(\sqrt{n} N)}\right)^{2} D_{2}^{-}+\left|\sum_{x}\left(\hat{x}-E_{\mathcal{D}(\sqrt{n} N)}\right)^{2} D_{2}^{+}-\sum_{x}\left(\hat{x}-E_{\mathcal{D}(\sqrt{n} N)}\right)^{2} D_{2}^{-}\right| \\
& \quad \leq C R_{h} R_{2}(N)(N) \lambda n N^{2} .
\end{aligned}
$$

Therefore,

$$
\begin{aligned}
\sum_{x} \| x & -E_{\mathcal{D}(\sqrt{n} N)}\left\|_{1}^{2}\left|D_{2}(x)\right| \leq(d-1) \sum_{x}\right\| x-E_{\mathcal{D}(\sqrt{n} N)} \|_{2}^{2}\left|D_{2}(x)\right| \\
& =(d-1) \sum_{i=2}^{d} \sum_{x}\left\langle x-E_{\mathcal{D}(\sqrt{n} N)}, e_{i}\right\rangle^{2}\left|D_{2}(x)\right| \leq(d-1)^{2} R_{h}(N) R_{2}(N) \lambda n N^{2} \\
& \leq R_{h+1}(N) \lambda n N^{2},
\end{aligned}
$$

proving (4).

\section{Reduction to quenched return probabilities}

\subsection{Basic calculations}

In this subsection we repeat a calculation from [9]. Our main goal is to control the probability of the event $\tau_{1}>u$. To this end, we take $L=\left\lceil(\log u)^{d / \gamma}\right\rceil$ and notice that

$$
\mathbb{P}\left(\tau_{1}>u\right) \leq \mathbb{P}\left(\tau_{1}>T_{L}\right)+\mathbb{P}\left(T_{L}>u\right) \leq e^{-(\log u)^{d}}+\mathbb{P}\left(T_{L}>u\right),
$$

where the last inequality follows from (4.1). Let $B_{L}:=[-L, L] \times\left[-L^{2}, L^{2}\right]^{d-1}$. Then, again by (4.1), $\mathbb{P}\left(T_{L} \neq T_{\partial B_{L}}\right) \leq e^{-(\log u)^{d}}$, and thus it is sufficient to show that

$$
\mathbb{P}\left(T_{\partial B_{L}}>u\right)<C e^{-c(\log u)^{\alpha}}
$$

for appropriate constants $C$ and $c$.

On the event $\left\{T_{\partial B_{L}}>u\right\}$, there exists a point $x \in B_{L}$ that is visited more than $u /\left|B_{L}\right|$ times before the walk leaves $B_{L}$. Therefore, it is sufficient to show that

$$
\mathbb{P}\left(\exists_{x \in B_{L}} T_{x}^{u /\left|B_{L}\right|}<T_{\partial B_{L}}\right)<C e^{-c(\log u)^{\alpha}},
$$

where $T_{x}^{k}$ is defined to be the $k^{\text {th }}$ hitting time of $x$. Let $G \subseteq \Omega$ be an event. Then

$$
\mathbb{P}\left(\exists_{x \in B_{L}} T_{x}^{u /\left|B_{L}\right|}<T_{\partial B_{L}}\right) \leq P\left(G^{c}\right)+\sup _{\omega \in G} P_{\omega}\left(\exists_{x \in B_{L}} T_{x}^{u /\left|B_{L}\right|}<T_{\partial B_{L}}\right),
$$


and

$$
\begin{aligned}
P_{\omega}\left(\exists_{x \in B_{L}} T_{x}^{u /\left|B_{L}\right|}<T_{\partial B_{L}}\right) \leq \sum_{x \in B_{L}} P_{\omega}^{0}\left(T_{x}^{u /\left|B_{L}\right|}<T_{\partial B_{L}}\right) \\
=\sum_{x \in B_{L}} P_{\omega}^{0}\left(T_{x}<T_{\partial B_{L}}\right) P_{\omega}^{x}\left(T_{x}^{u /\left|B_{L}\right|-1}<T_{\partial B_{L}}\right) \leq \sum_{x \in B_{L}} P_{\omega}^{x}\left(T_{x}^{u /\left|B_{L}\right|-1}<T_{\partial B_{L}}\right) .
\end{aligned}
$$

Note that due to the strong Markov property,

$$
P_{\omega}^{x}\left(T_{x}^{u /\left|B_{L}\right|-1}<T_{\partial B_{L}}\right)=\left[P_{\omega}^{x}\left(T_{x}<T_{\partial B_{L}}\right)\right]^{u /\left|B_{L}\right|-1},
$$

and therefore (5.1) will follow if we find an event $G$ such that $P\left(G^{c}\right)<\frac{1}{2} e^{-(\log u)^{\alpha}}$ and for some $\epsilon>0$, every $\omega \in G$ and every $x$,

$$
P_{\omega}^{x}\left(T_{\partial B_{L}}<T_{x}\right)>u^{\epsilon-1} .
$$

In turn, we may replace (5.2) by

$$
P_{\omega}^{x}\left(T_{\partial B_{2 L}(x)}<T_{x}\right)>u^{\epsilon-1},
$$

where $B_{2 L}(x)$ is the cube of the same dimensions as $B_{2 L}$, centered at $x$. The cube $B_{2 L}(x)$ is slightly more convenient than $B_{L}$ because now the condition is translation invariant with respect to the choice of $x$.

\subsection{Definition of the event $G$}

We now define the event $G$, and show that $P\left(G^{c}\right)<\frac{1}{2} e^{-(\log u)^{\alpha}}$. In Sections 6-8 we will show that (5.3) holds for every $\omega \in G$.

Let $\epsilon>0$ be such that

$$
2 d \epsilon<d-\alpha
$$

Fix $\psi>0$ such that

$$
\psi \leq \frac{\gamma \epsilon}{30 d}
$$

and $\chi>0$ such that

$$
\chi<\frac{\psi^{2}}{2} \cdot \frac{d-1}{2(d+1)} .
$$

We say that a basic block $\mathcal{P}(z, N)$ is good with respect to the environment $\omega$ if the assertion of Proposition 4.5 holds for every block of size at least $N^{\chi}$ that is contained in $\mathcal{P}(z, N)$, with $\theta=\psi / 2$. Otherwise, we say that $\mathcal{P}(z, N)$ is bad. We define our scales $N_{1}, \ldots, N_{\iota}$ as follows:

(1) $N_{1}:=\left\lceil L^{\psi}\right\rceil$.

(2) $\rho_{k}:=\chi / 2+\chi / 2^{k}$.

(3) $N_{k+1}:=N_{k} \cdot\left\lceil L^{\rho_{k}}\right\rceil$.

(4) $\iota$ is defined to be the largest $k$ such that $N_{k}^{2}<2 L$. 
For every $k=1, \ldots, \iota$, we let $B_{2 L}(k)$ be the set of all $z \in \mathcal{L}_{N_{k}}$ such that $\mathcal{P}\left(z, N_{k}\right) \cap B_{2 L}$ $\neq \emptyset$. We now define the event $G$ : We say that an environment $\omega$ is in $G$ if for every $k=1, \ldots, \iota$,

$$
\mid\left\{z \in B_{2 L}(k): \mathcal{P}\left(z, N_{k}\right) \text { is not good with respect to } \omega\right\} \mid<(\log u)^{\alpha+\epsilon} \text {. }
$$

Lemma 5.1. For $u$ large enough, $P(G) \geq 1-\frac{1}{2} e^{-(\log u)^{\alpha}}$.

Proof. Let

$$
J_{k}:=\mid\left\{z \in B_{2 L}(k): \mathcal{P}\left(z, N_{k}\right) \text { is not good with respect to } \omega\right\} \mid \text {. }
$$

First we note that

$$
P\left(G^{c}\right) \leq \sum_{k=1}^{\iota} P\left[J_{k} \geq(\log u)^{\alpha+\epsilon}\right],
$$

and $\iota$ is bounded. Now by Proposition 4.5 and Corollary 4.6, for given $k$ and $z \in B_{2 L}(k)$,

$$
p_{k}:=P\left(\mathcal{P}\left(z, N_{k}\right) \text { is not good }\right)=N_{k}^{-\xi(1)}=o\left(\left|B_{2 L}\right|^{-1}\right) .
$$

By Lemma 3.1, we can represent $J_{k}$ as $J_{k}=J_{k}^{(1)}+\cdots+J_{k}^{\left(9^{d}\right)}$, and

$$
J_{k}^{(h)} \sim \operatorname{Bin}\left(p_{k}, D_{k}\right)
$$

with $D_{k}<\left|B_{2 L}\right|$. Thus for $u$ large enough, $J_{k}^{(h)}$ is binomial with expected value less than 1 . Therefore, again assuming that $u$ is large enough,

$$
P\left[J_{k}^{(h)}>\frac{(\log u)^{\alpha+\epsilon}}{9^{d}}\right]<\exp \left(-\frac{(\log u)^{\alpha+\epsilon}}{9^{d}}\right) .
$$

Hence,

$$
P\left(G^{c}\right) \leq P\left(\bigcup_{k=1}^{\iota} \bigcup_{h=1}^{9^{d}} J_{k}^{(h)}\right) \leq 9^{d} \iota \exp \left(-\frac{(\log u)^{\alpha+\epsilon}}{9^{d}}\right) \leq \frac{1}{2} e^{-(\log u)^{\alpha}}
$$

\section{The auxiliary walk}

Fix an environment $\omega \in G$. In this section we define a new random walk $\left\{Y_{n}\right\}$ on the environment $\omega$, whose law is different from that of the quenched random walk $\left\{X_{n}\right\}$ on $\omega$. However, we show an obvious relation between the laws of $\left\{Y_{n}\right\}$ and $\left\{X_{n}\right\}$ that we will exploit in Sections 7 and 8 in order to prove (5.3).

We first give an informal description of $\left\{Y_{n}\right\}$ in Subsection 6.1, then define it properly in Subsection 6.2, and finally collect some useful facts about it in Subsection 6.3. 


\subsection{Informal description of $\left\{Y_{n}\right\}$}

$\left\{Y_{n}\right\}$ is a quenched random walk on $\omega$, which is forced to "behave well" in a number of different ways, which we list below.

(1) Once the walk $\left\{Y_{n}\right\}$ reaches the center of certain basic blocks, it is only allowed to exit them through their right boundaries.

(2) If the walk is in a bad block, then once it exists in the block, it is forced to make a number of steps on the right boundary of the block that will force the eventual exit distribution to be similar to the annealed distribution. We use Lemma 4.16 to control the number of forced steps needed. When the walk exits a good basic block, no such correction is necessary, because the distribution is already close enough to the annealed one.

(3) Upon leaving the origin the walk is forced to make a number of steps to the right. This together with (1) ensures that $\left\{Y_{n}\right\}$ leaves $B_{2 L}$ before returning to the origin.

The resulting $\left\{Y_{n}\right\}$ is a random walk that, most of the time, behaves locally similarly to the quenched random walk, but behaves globally similarly to the annealed random walk. We will quantify and then use those similarities in order to control the behavior of the quenched walk.

\subsection{Definition of $\left\{Y_{n}\right\}$}

The process $\left\{Y_{n}\right\}$ is a nearest neighbor random walk, which starts at 0 and stops when it reaches $\partial^{+} B_{2 L}$. Below we describe its law.

We first need some preliminary definitions. For all $j=1,2, \ldots$ and $k=1, \ldots, \iota$, we let $U_{k}(j)$ be the layer

$$
U_{k}(j)=H_{j N_{k}^{2}}=\left\{x:\left\langle x, e_{1}\right\rangle=j N_{k}^{2}\right\}
$$

We define $T_{k}^{Y}(j)=\inf \left\{n: Y_{n} \in U_{k}(j)\right\}$.

For all $x \in B_{2 L}$ and $k$, we define $z(x, k)$ as follows: if $\left\langle x, e_{1}\right\rangle$ is divisible by $N_{k}^{2}$, then $z(x, k)$ is a point $z \in \mathcal{L}_{N_{k}}$ such that $\left\langle x, e_{1}\right\rangle=\left\langle z, e_{1}\right\rangle$ and $x \in \tilde{\mathcal{P}}\left(z, N_{k}\right)$. If more than one such point exist, then we choose one according to some arbitrary rule. If $\left\langle x, e_{1}\right\rangle$ is not divisible by $N_{k}^{2}$, then we take $z(x, k)$ to be 0 .

For all $x \in B_{2 L}$ and $k$, we define $\mathcal{P}^{(k)}(x)=\mathcal{P}\left(z(x, k), N_{k}\right)$.

For every $x \in B_{2 L}$, we define

$$
k(x)=\max \left\{k \leq \iota:\left\langle x, e_{1}\right\rangle=\left\langle z(x, k), e_{1}\right\rangle \text { and } \mathcal{P}\left(z(x, k), N_{k}\right) \text { is good }\right\},
$$

and $k(x)=0$ if no such $k$ exists.

In addition, for a random variable $X$, a distribution $\mathcal{D}$ and a number $\lambda<1$, we define a $(\lambda, \mathcal{D})$-companion of $X$ as follows: Let $v$ be the distribution of $X$, and let $K$ be the smallest number such that $\nu$ is $(\lambda, K)$-close to $\mathcal{D}$. Let $\mu$ be an arbitrarily chosen coupling of three variables $Z_{0}, Z_{1}, Z_{2}$ demonstrating, as in Definition 11 , that $v$ is $(\lambda, k)$-close to $\mathcal{D}$. The roles of the variables $Z_{0}, Z_{1}, Z_{2}$ are exactly as in Definition 11 . In particular, 
$Z_{1} \sim \mathcal{D}$ and $Z_{2} \sim v$. We say that a variable $Y$ is a $(\lambda, \mathcal{D})$-companion of $X$ if the joint distribution of $X$ and $Y$ is the same as the $\mu$-joint distribution of $Z_{2}$ and $Z_{0}$. For all $X$, $\lambda$ and $\mathcal{D}$ we can construct such a companion: For every $x$, on the event $\{X=x\}$, we sample $Y$ according to the $\mu$-distribution of $Z_{0}$ conditioned on the event $\left\{Z_{2}=x\right\}$. Similarly, we can define the $(\lambda, \mathcal{D})$-companion of $X$ conditioned on a $\sigma$-algebra $\mathcal{F}$ : We work with the conditional distribution of $X$ given $\mathcal{F}$ instead of the (unconditional) distribution, and proceed as before. Note that $\|Y-X\|<K$ and that by Claim 4.7 the distribution of $Y$ is $(\lambda, 0)$-close to $\mathcal{D}$.

We now simultaneously define the walk $\left\{Y_{n}\right\}$, its accompanying sequence of times $\left\{\zeta_{m}\right\}$, and random variables $\beta_{k, j}$. The precise definition of the latter is postponed to the end of the subsection. However, we make the following comment on $\left\{\beta_{k, j}\right\}$ at this point: For every $j$ and $k$, a.s. $\left\langle\beta_{k, j}, e_{1}\right\rangle=0$.

For $j \leq N_{1}^{2}$, we define $Y_{j}=j e_{1}$. In addition, $\zeta_{0}=0$ and $\zeta_{1}=N_{1}^{2}$.

Given $\zeta_{0}, \ldots, \zeta_{n}$ and $\left\{Y_{\ell}: \ell=0, \ldots, \zeta_{n}\right\}$, we define $x^{\prime}=Y_{\zeta_{n}}$. Let $k^{\prime}$ be the largest $k$ such that $x^{\prime} \in U_{k}(j)$ for some $j$. Then we let $x=x^{\prime}+\sum_{k=1}^{k^{\prime}} \beta_{k, j(k)}$, where $j(k)$ is the value of $j$ such that $x^{\prime} \in U_{k}(j)$. We let $\kappa=\left\|x^{\prime}-x\right\|_{1}+2$ and choose $\left\{Y_{\zeta_{n}}, \ldots, Y_{\zeta_{n}+\kappa-2}\right\}$ to be a shortest path from $x^{\prime}$ to $x$. We then take $Y_{\zeta_{n}+\kappa-1}=x+e_{1}$ and $Y_{\zeta_{n}+\kappa}=x$. Let $\zeta_{n}^{\prime}=\zeta_{n}+\kappa$.

Let $k=k(x)$. If $k(x)>0$ then $\left\{Y_{\ell}: \ell=\zeta_{n}^{\prime}, \ldots, T_{\partial \mathcal{P}^{(k)}(x)}\right\}$ is chosen to be a random walk starting at $x$ on the random environment $\omega$ conditioned on the event $\left\{T_{\partial \mathcal{P}^{(k)}(x)}=T_{\partial^{+} \mathcal{P}^{(k)}(x)}\right\}$ and $\zeta_{n+1}=T_{\partial^{+} \mathcal{P}^{(k)}(x)}$. Conditioned on $\omega, \zeta_{n}^{\prime}$ and $x$, the path $\left\{Y_{\ell}: \ell=\zeta_{n}^{\prime}, \ldots, T_{\partial \mathcal{P}^{(k)}(x)}\right\}$ is chosen independently of the path prior to $\zeta_{n}^{\prime}$ and of $\left\{\beta_{k, j(k)}: k\right.$ and $j$ are such that $\left.j N_{k}^{2} \leq\left\langle x, e_{1}\right\rangle\right\}$. If $k=0$ then $\zeta_{n+1}=\zeta_{n}^{\prime}+N_{1}^{2}$ and for $\zeta_{n}^{\prime}<j \leq \zeta_{n+1}$, we take $Y_{j}=x+\left(j-\zeta_{n}^{\prime}\right) e_{1}$.

We define $x_{n}^{\prime}=Y_{\zeta_{n}}$ and $x_{n}=Y_{\zeta_{n}^{\prime}}$. Note that for every $n$, both $\left\langle x_{n}, e_{1}\right\rangle$ and $\left\langle x_{n}^{\prime}, e_{1}\right\rangle$ are divisible by $N_{1}^{2}$ (remember that $\left\langle\beta_{k, j}, e_{1}\right\rangle=0$ ).

All that is now left is to define $\beta_{k, j}$. First $\beta_{1,1}$ is simply defined to be the $\left(0, \mathcal{D}\left(N_{1}\right)\right)$ companion of the (deterministic) variable $Y_{N_{1}^{2}}$.

For other values of $k$ and $j$, we first list two conditions under which $\beta_{k, j}$ is zero.

- If there exist no $n$ such that $\zeta_{n}=T_{k}^{Y}(j-1)$ then $\beta_{k, j}=0$.

- Otherwise, let $n$ be such that $\zeta_{n}=T_{k}^{Y}(j-1)$, and let $x=Y_{\zeta_{n}^{\prime}}$. If $\mathcal{P}^{(k)}(x)$ is good, then $\beta_{k, j}=0$.

Now assume that neither of these conditions holds. For $k=1, \ldots, \iota$ let $\lambda_{k}=$ $L^{-\chi} R_{5+k}(L)$.

We define $\beta_{k, j}$ recursively—we use the values of $\left\{\beta_{k^{\prime}, j^{\prime}}: k^{\prime}<k, j^{\prime}=j N_{k}^{2} / N_{k^{\prime}}^{2}\right\}$ in the definition of $\beta_{k, j}$.

Let $x=Y_{\zeta_{n}^{\prime}}$, where as before $n$ is such that $\zeta_{n}=T_{k}^{Y}(j-1)$, and for $k^{\prime}<k$ let $j\left(k^{\prime}\right)$ be the unique value satisfying $U_{k^{\prime}}\left(j\left(k^{\prime}\right)\right)=U_{k}(j)$. Let

$$
X=Y_{T_{k}^{Y}(j)}-x+\sum_{k^{\prime}=1}^{k-1} \beta_{k^{\prime}, j\left(k^{\prime}\right)}
$$


Recall the definition of $\mathcal{D}(N)$ from page 154. Then $\mathcal{D}\left(N_{k}\right)$ is the annealed distribution of $X_{T_{k}(j)}-x$ for a walk starting at $x$, conditioned on exiting $\mathcal{P}\left(x, N_{k}\right)$ through the front.

We now take $\hat{Z}$ to be an (arbitrarily chosen) $\left(\lambda_{k}, \mathcal{D}\left(N_{k}\right)\right)$-companion of $X$, conditioned on $\left\{Y_{\ell}: \ell=1, \ldots, \zeta_{n}^{\prime}\right\}$ and $\omega$, and let $\beta_{k, j}=\hat{Z}-X$.

Thus we have defined the process $\left\{Y_{n}\right\}$.

Remark 1. Note that in our definition, if $\beta_{k, j} \neq 0$ then the distribution of $\hat{Z}=X+\beta_{k, j}$ is $\left(\lambda_{k}, 0\right)$-close to $\mathcal{D}\left(N_{k}\right)$.

\subsection{Basic properties of $\left\{Y_{n}\right\}$}

We prove a few facts regarding the process $\left\{Y_{n}\right\}$ which we will use in Sections 7 and 8.

Lemma 6.1. $\left\{Y_{n}\right\}$ reaches $\partial B_{L}$ before returning to the origin.

Proof. By the definition, $U_{1}(1)$ is reached before returning to the origin. Then for all $n$, if $x=Y_{\zeta_{n}}$, then $\mathcal{P}^{(k(x))}(x)$ is contained in the positive half-space, and $\left\{Y_{n}\right\}$ exits $\mathcal{P}^{(k(x))}(x)$ through $\partial^{+} \mathcal{P}^{(k)}(x)$. Therefore $\left\{Y_{n}\right\}$ cannot return to the origin.

Lemma 6.2. For every $k$ and $j$, with probability 1 ,

$$
\beta_{k, j}<L^{4 \psi} \text {. }
$$

Proof. For $k=1$, the size of the block $\mathcal{P}\left(0, N_{1}\right)$ is less than $L^{4 \psi}$, and therefore for every $j$, we have $\beta_{1, j}<L^{4 \psi}$.

Now assume that $k \geq 1$. In this case, we assume that there exists $n$ such that $\zeta_{n}=$ $T_{k}^{Y}(j-1)$, because otherwise $\beta_{k, j}=0$. Let $i$ be such that $U_{k}(j-1)=U_{k-1}(i)$.

Let $x=Y_{\zeta_{n}^{\prime}}$. If $\mathcal{P}^{(k)}(x)$ is good, then $\beta_{k, j}=0$. Therefore we may assume that $\mathcal{P}^{(k)}(x)$ is not good. In this case there exist $n_{0}=n, n_{1}, \ldots, n_{m}$ such that $m$ satisfies $U_{k}(j)=U_{k-1}(i+m)$ and for $h=0,1, \ldots, m$, we have $\zeta_{n_{h}}=T_{k-1}^{Y}(i+h)$.

For $1 \leq h<m$, let $X_{h}=Y_{\zeta_{n_{h}}^{\prime}}-Y_{\zeta_{n_{h-1}}^{\prime}}$, and let $X_{m}=Y_{\zeta_{n_{m}}^{\prime}}-Y_{\zeta_{n_{m-1}}^{\prime}}-\beta_{k, j}$. We now claim that for every $1 \leq h \leq m$, conditioned on $X_{1}, \ldots, X_{h-1}$, the distribution of $X_{h}$ is $\left(\lambda_{k-1}, N_{k-1}^{\psi / 2}\right)$-close to $\overline{\mathcal{D}}\left(N_{k-1}\right)$. Indeed, if $\mathcal{P}^{(k-1)}\left(Y_{\zeta_{n-1}^{\prime}}\right)$ is good, then this claim follows from Corollary 4.6. Otherwise, as in Remark 1, the distribution of $X_{h}$ is $\left(\lambda_{k-1}, 0\right)$-close to $\mathcal{D}\left(N_{k-1}\right)$ (and in particular $\left(\lambda_{k-1}, N_{k-1}^{\psi / 2}\right)$-close to $\mathcal{D}\left(N_{k-1}\right)$ ).

Therefore, by Lemma 4.16, the distribution of

$$
Y_{T_{k}^{Y}(j)}-x+\sum_{k^{\prime}=1}^{k-1} \beta_{k^{\prime}, j\left(k^{\prime}\right)}=\sum_{h=1}^{m} X_{h}
$$

is $\left(\lambda_{k}, R_{k+6}(L) N_{k-1}^{\psi / 2} N_{k}^{2} / N_{k-1}^{2}\right)$-close to $\mathcal{D}\left(N_{k}\right)$. Hence, with probability 1 ,

$$
\beta_{k, j} \leq N_{k-1}^{\psi / 2} \cdot \frac{R_{k+6}(L) N_{k}^{2}}{N_{k-1}^{2}} \leq L^{4 \psi}
$$


Lemma 6.3. For $j$ and $k$, if there exists $n$ such that $x_{n} \in U_{k}(j)$, then at least one of the following holds:

(1) There exists $j^{\prime}$ such that $U_{k}(j)=U_{l}\left(j^{\prime}\right)$.

(2) There exist $k^{\prime}$ and $j^{\prime}$ such that $U_{k}(j)=U_{k^{\prime}}\left(j^{\prime}\right)$ and $x_{n-1} \in U_{k^{\prime}}\left(j^{\prime}-1\right)$ and $x_{n-1}$ is contained in a block $\mathcal{P}\left(z, N_{k^{\prime}+1}\right)$ such that $z \in \mathcal{L}_{N_{k^{\prime}+1}}$ and $\mathcal{P}\left(z, N_{k^{\prime}+1}\right)$ is not good.

(3) $j \leq\left(N_{k+1} / N_{k}\right)^{2}$.

Proof. Assume that $x_{n} \in U_{k}(j)$. Let $k^{\prime}=k\left(x_{n-1}\right)$. If $k^{\prime}=0$ then case (2) holds. Assume $k^{\prime}>0$. Then the intersection of $U_{k}(j)$ and $\partial^{+} \mathcal{P}^{\left(k^{\prime}\right)}\left(x_{n-1}\right)$ is not empty. Therefore, by the definition of $k(x)$, there is some $j^{\prime}$ such that $U_{k}(j)=U_{k^{\prime}}\left(j^{\prime}\right)$ and $x_{n-1} \in U_{k^{\prime}}\left(j^{\prime}-1\right)$, and one of the following occurs:

- $k^{\prime}=\imath$.

- There exists $z \in \mathcal{L}_{N_{k^{\prime}+1}}$ such that $\left\langle z, e_{1}\right\rangle=\left\langle x_{n-1}, e_{1}\right\rangle$, and $\mathcal{P}^{\left(k^{\prime}+1\right)}\left(x_{n-1}\right)$ is not good.

- No $z \in \mathcal{L}_{N_{k^{\prime}+1}}$ exists with $\left\langle z, e_{1}\right\rangle=\left\langle x_{n-1}, e_{1}\right\rangle$.

In the first two cases, the lemma holds. Thus we assume that the last case holds.

Then there exists $n^{\prime}<n-1$ such that $\left\langle x_{n^{\prime}}, e_{1}\right\rangle=N_{k^{\prime}+1}^{2}\left\lfloor\left\langle x_{n-1}, e_{1}\right\rangle / N_{k^{\prime}+1}^{2}\right\rfloor$. Now, $x_{n-1}$ is in $\mathcal{P}^{\left(k^{\prime}+1\right)}\left(x_{n^{\prime}}\right)$. If $\mathcal{P}^{\left(k^{\prime}+1\right)}\left(x_{n^{\prime}}\right)$ is not good, then $x_{n-1}$ is contained in a block $\mathcal{P}\left(z, N_{k^{\prime}+1}\right)$ such that $z \in \mathcal{L}_{N_{k^{\prime}+1}}$ and $\mathcal{P}\left(z, N_{k^{\prime}+1}\right)$ is not good. If $\mathcal{P}^{\left(k^{\prime}+1\right)}\left(x_{n^{\prime}}\right)$ is good and $\left\langle x_{n^{\prime}}, e_{1}\right\rangle \neq 0$ then $\zeta_{n^{\prime}+1}$ is the exit time from $\mathcal{P}^{\left(k^{\prime}+1\right)}\left(x_{n^{\prime}}\right)$, contradicting the assumptions. If $\left\langle x_{n^{\prime}}, e_{1}\right\rangle=0$, then $j \leq\left(N_{k+1} / N_{k}\right)^{2}$.

We now let $M$ be the number of stopping times $\zeta_{n}$ in the definition of $\left\{Y_{n}\right\}$.

Lemma 6.4. Let $[Y]$ be the set of points visited by $\left\{Y_{n}\right\}$. For every $k=1, \ldots$, , let

$$
Q_{k}\left(\left\{Y_{n}\right\}\right)=\#\left\{z \in \mathcal{L}_{N_{k}}:[Y] \cap \mathcal{P}\left(z, N_{k}\right) \neq \emptyset \text { and } \mathcal{P}\left(z, N_{k}\right) \text { is bad }\right\} .
$$

Then

$$
M \leq \frac{2 L}{N_{\iota}^{2}}+L^{2 \chi} \sum_{k=1}^{\iota} Q_{k}\left(\left\{Y_{n}\right\}\right)+\iota L^{2 \chi} \leq L^{2 \chi} \cdot\left(\iota+2+\sum_{k=1}^{\iota} Q_{k}\left(\left\{Y_{n}\right\}\right)\right) .
$$

Proof. This follows from Lemma 6.3. There are at most $2 L / N_{l}^{2}$ stopping times that are caused by reaching the end of an $N_{\iota}$ block, $\iota L^{2 \chi}$ stopping times that are caused by the beginning and at most $L^{2 \chi} \sum_{k=1}^{\iota} Q_{k}\left(\left\{Y_{n}\right\}\right)$ stopping times that are caused by visiting blocks that are not good.

We now draw a connection between the walks $\left\{Y_{n}\right\}$ and $\left\{X_{n}\right\}$.

Lemma 6.5. Let $v=\left(v_{1}, \ldots, v_{N_{v}}\right)\left(N_{v}\right.$ is the length of the path $\left.v\right)$ be a nearestneighbor path starting at the origin, never returning to the origin, and ending at $\partial^{+} B_{L}$. For every $k=1, \ldots, \iota$, let

$$
Q_{k}(v)=\#\left\{z \in \mathcal{L}_{N_{k}}: v \cap \mathcal{P}\left(z, N_{k}\right) \neq \emptyset \text { and } \mathcal{P}\left(z, N_{k}\right) \text { is bad }\right\},
$$


and let $Q(v)=L^{2 \chi} \cdot\left(\iota+2+\sum_{k=1}^{\iota} Q_{k}(v)\right)$. Then

$$
\frac{P_{\omega}\left(X_{j}=v_{j} \text { for all } j<N_{v}\right)}{P_{\omega}\left(Y_{j}=v_{j} \text { for all } j<N_{v}\right)} \geq \frac{1}{2} \eta^{Q(v) \cdot\left(L^{4 \psi}+2\right)},
$$

where $\eta$ is the ellipticity constant, as in (1.1).

Proof. First note that due to uniform ellipticity,

$$
P_{\omega}\left(X_{j}=v_{j} \text { for all } j<N_{v}\right)>0
$$

for every $v$. Therefore without loss of generality we can restrict ourselves to considering only $v$ 's such that

$$
P_{\omega}\left(Y_{j}=v_{j} \text { for all } j<N_{v}\right)>0 .
$$

For such $v$, we define the sequences of times $\zeta_{n}$ and $\zeta_{n}^{\prime}$ in a fashion very similar to the definition in the construction of the $Y$-process: $\zeta_{0}=\zeta_{0}^{\prime}=0$ and $\zeta_{1}=N_{1}^{2}$. Given $\zeta_{0}, \ldots, \zeta_{n}$ and $\zeta_{0}^{\prime}, \ldots, \zeta_{n-1}^{\prime}$, let $x_{n}^{\prime}=v_{\zeta_{n}}$. Let $\zeta_{n}^{\prime}$ be the smallest $\ell>\zeta_{n}$ such that $\left\langle v_{\ell-1}, e_{1}\right\rangle>$ $\left\langle x_{n}^{\prime}, e_{1}\right\rangle$, and let $x_{n}=v_{\zeta_{n}^{\prime}}$. Let $k=k\left(x_{n}\right)$. If $k>0$, then we let $\zeta_{n+1}=T_{\partial^{+}} \mathcal{P}^{(k)}\left(x_{n}\right)(v)$.

Otherwise, $\zeta_{n+1}=\zeta_{n}^{\prime}+N_{1}^{2}$. Then

$$
\begin{aligned}
& P_{\omega}\left(Y_{j}=v_{j} \text { for all } j<N_{v}\right) \\
& \quad \leq \prod_{n: k\left(x_{n}\right)>0} P_{\omega}^{x_{n}}\left(X_{\ell}=v_{\ell+\zeta_{n}^{\prime}} ; \ell=1, \ldots, \zeta_{n+1}-\zeta_{n}^{\prime} \mid T_{\partial \mathcal{P}^{(k)}\left(x_{n}\right)}=T_{\partial+\mathcal{P}^{(k)}\left(x_{n}\right)}\right),
\end{aligned}
$$

and

$$
\begin{array}{r}
P_{\omega}\left(X_{j}=v_{j} \text { for all } j<N_{v}\right) \\
\geq \prod_{n: k\left(x_{n}\right)>0} P_{\omega}^{x_{n}}\left(X_{\ell}=v_{\ell+\zeta_{n}^{\prime}} ; \ell=1, \ldots, \zeta_{n+1}-\zeta_{n}^{\prime} \mid T_{\partial \mathcal{P}^{(k)}\left(x_{n}\right)}=T_{\partial^{+} \mathcal{P}^{(k)}\left(x_{n}\right)}\right) \\
\cdot \prod_{n: k\left(x_{n}\right)>0} P_{\omega}^{x_{n}}\left(T_{\partial \mathcal{P}^{(k)}(x)}=T_{\partial^{+} \mathcal{P}^{(k)}\left(x_{n}\right)}\right) \\
\cdot \prod_{n} \eta^{\left\|x_{n}^{\prime}-x_{n}\right\|_{1}+2} \cdot \prod_{n: k\left(x_{n}\right)=0} \eta^{L^{2 \psi}} .
\end{array}
$$

The first inequality follows from the fact that inside the good blocks, $\left\{Y_{n}\right\}$ performs quenched random walk on the environment $\omega$. For the second inequality, the first term and (6.4) count the probability of all steps in the good blocks. In addition, at each stopping time, the process $\left\{X_{n}\right\}$ has to walk from $x_{n}^{\prime}$ to $x_{n}$, and when $k\left(x_{n}\right)=0$ it also needs to traverse through an $N_{1}$ block. In (6.5) we bound the probability of all of these steps by ellipticity.

By Proposition 4.5, the product in (6.4) is no less than 1/2. By the definitions of $k(x)$ and $\beta_{k, j}$, by Lemma 6.2, and by uniform ellipticity with constant $\eta$, the product in (6.5) is bounded below by $\eta^{Q(v) \cdot\left(L^{4 \psi}+2\right)}$. Therefore,

$$
\frac{P_{\omega}\left(X_{j}=v_{j} \text { for all } j<N_{v}\right)}{P_{\omega}\left(Y_{j}=v_{j} \text { for all } j<N_{v}\right)} \geq \frac{1}{2} \eta^{Q(v) \cdot\left(L^{4 \psi}+2\right)} .
$$


For any $k$ and $j$, we define $T_{k}^{\prime Y}(j)$ as follows: If there exists $n$ such that $\zeta_{n}=T_{k}^{Y}(j)$, then $T_{k}^{\prime Y}(j)=\zeta_{n}^{\prime}$. Otherwise, $T_{k}^{\prime Y}(j)=T_{k}^{Y}(j)$.

Lemma 6.6. Conditioned on $\left\{Y_{\ell}: \ell \leq T_{k}^{\prime Y}(j-1)\right\}$, the distribution of $Y_{T_{k}^{\prime Y}(j)}$ $Y_{T_{k}^{\prime Y}(j-1)}$ is $\left(\lambda_{l}, 2 L^{4 \psi}\right)$-close to $\mathcal{D}\left(N_{k}\right)$.

Proof. We look at two different cases: If $\mathcal{P}^{(k)}\left(Y_{T_{k}^{\prime Y}(j-1)}\right)$ is good, then the conclusion follows from Corollary 4.6. Otherwise, it follows from the definition of $\beta_{k, j}$.

From Lemma 6.6, we get the following useful corollary.

Corollary 6.7. Assume that $u$ is large enough. Condition on $\left\{Y_{\ell}: \ell \leq T_{k}^{\prime Y}(j-1)\right\}$, and let $\bar{Y}=Y_{T_{k}^{\prime Y}(j-1)}+\mathbb{E}\left(X_{T_{N_{k}^{2}}}\right)$. For every $x \in U_{k}(j)$ such that $\|x-\bar{Y}\|<4 N_{k}$,

$$
P_{\omega}\left(\left\|Y_{T_{k}^{\prime Y}(j)}-x\right\|<N_{k} \mid Y_{\ell}, \ell \leq T_{k}^{\prime Y}(j-1)\right)>\rho
$$

for some constant $\rho>0$.

Proof. By Lemma 6.6, the quenched distribution of $Y_{T_{k}^{\prime Y}(j)}-Y_{T_{k}^{\prime Y}(j-1)}$ conditioned on the history of the walk is $\left(\lambda_{l}, 2 L^{4 \psi}\right)$-close to the annealed distribution $\mathcal{D}\left(N_{k}\right)$. Therefore,

$$
P_{\omega}\left(\left\|Y_{T_{k}^{\prime Y}(j)}-x\right\|<N_{k} \mid Y_{\ell}, \ell \leq T_{k}^{\prime Y}(j-1)\right)>\mathcal{D}\left(N_{k}\right)\left(y:\|y-x\|<N_{k} / 2\right)-\lambda_{l} .
$$

By Lemma 4.4, $\mathcal{D}\left(N_{k}\right)\left(y:\|y-x\|<N_{k} / 2\right)$ is bounded away from zero. On the other hand, $\lambda_{\iota}$ goes to zero as $L$ goes to infinity. The corollary follows.

Lemma 6.8. Conditioned on $\left\{Y_{\ell}: \ell \leq T_{k}^{\prime Y}(j-1)\right\}$, the (quenched) probability that $\left\{Y_{\ell}\right\}_{\ell \geq T_{k}^{\prime Y}(j-1)}$ exits $\mathcal{P}^{(k)}\left(Y_{T_{k}^{\prime Y}(j-1)}\right)$ through $\partial^{+} \mathcal{P}^{(k)}\left(Y_{T_{k}^{\prime Y}(j-1)}\right)$ is $1-L^{-\xi(1)}$.

Proof. We denote by $E$ the event whose probability we are trying to estimate. If $\mathcal{P}^{(k)}\left(Y_{T_{k}^{\prime Y}(j-1)}\right)$ is good, then the conclusion follows by the definition of a good block. Therefore we may assume that $\mathcal{P}^{(k)}\left(Y_{T_{k}^{\prime Y}(j-1)}\right)$ is a bad block. In this case we use induction on $k$. For $k=1$ the conclusion follows immediately from the definition of the auxiliary walk on bad $N_{1}$ blocks.

Now assume $k>1$. We assume that the conclusion holds for $Y_{T_{k-1}^{\prime Y}(h)}$ for every $h$ (if the block $\mathcal{P}^{(k-1)}\left(Y_{T_{k-1}^{\prime Y}(h-1)}\right)$ is good, then we already proved it; if the block is bad then this is the induction hypothesis).

Let $l$ be such that $l N_{k-1}^{2}=(j-1) N_{k}^{2}$, and let $m$ be such that $(l+m) N_{k-1}^{2}=j N_{k}^{2}$ For $h=1, \ldots, m$, let

$$
I_{h}=Y_{T_{k-1}^{\prime Y}(l+h)}-Y_{T_{k-1}^{\prime Y}(l+h-1)} .
$$

Let $A$ be the event that for every $h=1, \ldots, m$, the walk $\left\{Y_{\ell}\right\}$ leaves $\mathcal{P}^{(k-1)}\left(Y_{T_{k-1}^{\prime}(j-1)}\right)$ through its front. Then by the induction hypothesis, $P_{\omega}\left(A \mid Y_{\ell}, \ell=1, \ldots, T_{k}^{\prime Y}(j-1)\right)=$ $1-L^{-\xi(1)}$. 
Now,

$$
\begin{aligned}
& P_{\omega}\left(E^{c} \mid Y_{\ell}, \ell=1, \ldots, T_{k}^{\prime Y}(j-1)\right) \\
& \quad \leq P_{\omega}\left(A^{c} \mid Y_{\ell}, \ell=1, \ldots, T_{k}^{\prime Y}(j-1)\right)+P_{\omega}\left(E^{c} \mid A ; Y_{\ell}, \ell=1, \ldots, T_{k}^{\prime Y}(j-1)\right),
\end{aligned}
$$

and

$$
\begin{aligned}
& P_{\omega}\left(\left.E^{c}\right|_{Y_{\ell}, \ell=1, \ldots, T_{k}^{\prime Y}(j-1)}\right) \\
& \leq P_{\omega}\left(\exists_{1 \leq h \leq m}\left\|\sum_{i=1}^{h} I_{i}-h N_{k-1}^{2} \frac{\vartheta}{\left\langle\vartheta, e_{1}\right\rangle}\right\|>\left.\frac{1}{2} N_{k} R_{5}\left(N_{k}\right)\right|_{Y_{\ell}, \ell=1, \ldots, T_{k}^{\prime Y}(j-1)}\right) \\
& \leq \sum_{h=1}^{m} P_{\omega}\left(\left\|\sum_{i=1}^{h} I_{i}-h N_{k-1}^{2} \frac{\vartheta}{\left\langle\vartheta, e_{1}\right\rangle}\right\|>\left.\frac{1}{2} N_{k} R_{5}\left(N_{k}\right)\right|_{Y_{\ell}, \ell=1, \ldots, T_{k}^{\prime Y}(j-1)}\right) .
\end{aligned}
$$

It is sufficient to show that for every $h$, the probability in (6.7) is $L^{-\xi(1)}$.

Fix $h$. Conditioned on $A$, the variable $J_{i}=I_{i}-N_{k-1}^{2} \vartheta /\left\langle\vartheta, e_{1}\right\rangle$ is bounded by $2 N_{k-1} R_{5}\left(N_{k-1}\right)$. Furthermore, the quenched expectation of $J_{i}$ conditioned on $A, J_{1}, \ldots$, $J_{i-1}$ and $Y_{\ell}, \ell=1, \ldots, T_{k}^{\prime Y}(j-1)$, is bounded by $N_{k-1}^{\psi / 2}$ (see (5.5)).

Therefore, using the Azuma-Höffding inequality, we get

$$
\begin{aligned}
& P_{\omega}\left(\left.E^{c}\right|_{Y_{\ell}, \ell=1, \ldots, T_{k}^{\prime Y}(j-1)}\right) \\
& \leq C \exp \left(\frac{-N_{k}^{2} R_{5}\left(N_{k}\right)^{2}}{8 N_{k-1}^{2} R_{5}\left(N_{k-1}\right)^{2} \cdot\left(N_{k} / N_{k}-1\right)^{2}}\right)=C \exp \left(\frac{-R_{5}\left(N_{k}\right)^{2}}{8 R_{5}\left(N_{k-1}\right)^{2}}\right) \\
& \leq C_{1} \exp \left(-C_{2} \exp \left(\left[\log \left(\rho_{1}+\cdots+\rho_{k-1}+\rho_{k}\right)-\log \left(\rho_{1}+\cdots+\rho_{k-1}\right)\right][\log \log L]^{5}\right)\right) \\
& =L^{-\xi(1)},
\end{aligned}
$$

where the last inequality follows from the definition of $N_{k}$, the definition of $R_{k}(N)$, and a first order Taylor approximation.

\section{The random direction event}

In this section we consider an event $W^{(w)}$ which we call the random direction event. First we construct it, and then show that the probability that $W^{(w)}$ occurs is more than $u^{\epsilon-1 / 2}$. Next we show some estimates on the hitting probabilities of the walk conditioned on the occurrence of $W^{(w)}$. In the next section we will show that these estimates are sufficient for proving (5.3), and thus Theorem 1.5.

\subsection{Definition of $W^{(w)}$}

Let $M=\left[(\log u)^{1-\epsilon}\right]$, and for $k=1, \ldots, \iota$ let $\mathcal{E}_{k}=\mathbb{E}^{0}\left(X_{T_{\partial} \mathcal{P}\left(0, N_{k}\right)} \mid T_{\partial \mathcal{P}\left(0, N_{k}\right)}=\right.$ $T_{\partial}+\mathcal{P}\left(0, N_{k}\right)$ be the annealed expectation of the point of exit of $\mathcal{P}\left(0, N_{k}\right)$. Let $A_{1}=1$, 
and for every $k>1$, let $A_{k}$ be the smallest integer such that $A_{k} N_{k}^{2}>\left(M+A_{k-1}\right) N_{k-1}^{2}$. Note that $A_{k} \leq M$.

For $k=1, \ldots, \iota$ and $j>A_{k}$, we define $\mathbb{B}_{k}(j)$ to be the event that $\left\{Y_{n}\right\}$ leaves $\mathcal{P}^{(k)}\left(Y_{T_{k}^{\prime Y}(j-1)}\right)$ through $\partial^{+} \mathcal{P}^{(k)}\left(Y_{T_{k}^{\prime Y}(j-1)}\right)$.

Fix $w \in[-1,1]^{d-1}$. For $j>A_{k}$ we define the event $W_{k}^{(w)}(j)$ as follows:

$$
W_{k}^{(w)}(j)=\left\{\left\|Y_{T_{k}^{\prime Y}(j)}-Y_{T_{k}^{\prime Y}\left(A_{k}\right)}-\left(j-A_{k}\right) \mathcal{E}_{k}-w\left(j-A_{k}\right) N_{k}\right\|<N_{k}\right\} .
$$

Then

$$
W_{k}^{(w)}=\bigcap_{j=A_{k}+1}^{A_{k}+M}\left[W_{k}^{(w)}(j) \cap \mathbb{B}_{k}(j)\right],
$$

and $W^{(w)}$ is defined to be the intersection

$$
W^{(w)}=\bigcap_{k=1}^{\iota} W_{k}^{(w)} .
$$

7.2. The probability of $W^{(w)}$

In this subsection we bound from below the probability of the event $W^{(w)}$.

Lemma 7.1. (1) There exists some $\rho>0$ such that for $1 \leq k \leq \iota$ and $A_{k}<j \leq A_{k}+M$,

$$
\begin{aligned}
P_{\omega}\left(W_{k}^{(w)}(j) \mid W_{1}^{(w)}, \ldots, W_{k-1}^{(w)}, W_{k}^{(w)}\left(A_{k}+1\right), \ldots,\right. \\
\left.W_{k}^{(w)}(j-1), \mathbb{B}_{k}\left(A_{k}+1\right), \ldots, \mathbb{B}_{k}(j-1)\right)>\rho .
\end{aligned}
$$

(2) For $1 \leq k \leq \iota$ and $A_{k}<j \leq A_{k}+M$,

$$
\begin{aligned}
P_{\omega}\left(\mathbb{B}_{k}(j) \mid W_{1}^{(w)}, \ldots, W_{k-1}^{(w)}, W_{k}^{(w)}\left(A_{k}+1\right), \ldots,\right. \\
\left.W_{k}^{(w)}(j-1), \mathbb{B}_{k}\left(A_{k}+1\right), \ldots, \mathbb{B}_{k}(j-1)\right)=1-o(1) .
\end{aligned}
$$

Proof. For (1), conditioned on $W_{1}^{(w)} \cap \cdots \cap W_{k-1}^{(w)} \cap W_{k}^{(w)}\left(A_{k}+1\right) \cap \cdots \cap W_{k}^{(w)}(j-1)$, $\mathbb{B}_{k}\left(A_{k}+1\right), \ldots, \mathbb{B}_{k}(j-1)$, we get

$$
\left\|Y_{T_{k}^{\prime Y}(j-1)}-Y_{T_{k}^{\prime Y}\left(A_{k}\right)}-(j-1) \mathcal{E}_{k}-w(j-1) N_{k}\right\|<N_{k} .
$$

Therefore,

$$
\left\|Y_{T_{k}^{\prime Y}\left(A_{k}\right)}+j \mathcal{E}_{k}+w j N_{k}-\left(Y_{T_{k}^{\prime Y}(j-1)}+\mathcal{E}_{k}\right)\right\|<4 N_{k} .
$$

By Corollary 6.7 and the definition of $W_{k}(j)$, we get the conclusion of (1).

Part (2) follows from Lemma 6.8.

As a consequence of Lemma 7.1 and the choice of $M$, we get the following lemma:

Lemma 7.2. The probability of $W^{(w)}$ is bounded from below by $u^{\epsilon-1 / 2}$. 


\subsection{Hitting probability estimates}

In this subsection we bound from above the probability, conditioned on $W^{(w)}$, of a block to be hit. We begin with a simple claim.

Claim 7.3. Fix $k$ between 1 and $\iota$, and let

$$
A_{k}+M \leq j \leq\left(\frac{N_{k+1}}{N_{k}}\right)^{2}\left(A_{k+1}+M\right) .
$$

Let $z \in \mathcal{L}_{N_{k}} \cap U_{k}(j)$. Then

$$
\int_{[-1,1]^{d-1}} P_{\omega}\left(\left\{Y_{n}\right\} \cap \mathcal{P}\left(z, N_{k}\right) \neq \emptyset \mid W^{(w)}\right) d w \leq(\log u)^{(1-d)(1-2 \epsilon)} .
$$

Proof. First note that there exist $A_{k+1}<j^{\prime} \leq A_{k+1}+M$ and $z^{\prime} \in \mathcal{L}_{N_{k+1}} \cap U_{k+1}\left(j^{\prime}\right)$ such that $\mathcal{P}\left(z, N_{k}\right) \subseteq \mathcal{P}\left(z^{\prime}, N_{k+1}\right)$. Then by the definition of $W^{(w)}$ (and using the fact that $W^{(w)}$ implies $\left.\mathbb{B}_{k+1}\left(j^{\prime}\right)\right)$, the probability

$$
P_{\omega}\left(\left\{Y_{n}\right\} \cap \mathcal{P}\left(z, N_{k}\right) \neq \emptyset \mid W^{(w)}\right)
$$

is positive only if

$$
\left\|z^{\prime}-M \mathcal{E}_{k}-j^{\prime} \mathcal{E}_{k+1}-M w N_{k}-j^{\prime} w N_{k+1}\right\|<N_{k+1} R_{5}\left(N_{k+1}\right)
$$

and in particular $w$ needs to be in an area of side length which is no more than $N_{k+1} R_{5}\left(N_{k+1}\right) / M N_{k} \leq M^{-1} L^{\chi}$ and thus the integral in (7.1) is bounded by $(\log u)^{(1-d)(1-2 \epsilon)}$.

Lemma 7.4. Fix $k$ between 1 and , and let $j>A_{k}+M$. Let $z \in \mathcal{L}_{N_{k}} \cap U_{k}(j)$. Then

$$
\int_{[-1,1]^{d-1}} P_{\omega}\left(\left\{Y_{n}\right\} \cap \mathcal{P}\left(z, N_{k}\right) \neq \emptyset \mid W^{(w)}\right) d w \leq(\log u)^{(1-d)(1-2 \epsilon)} .
$$

Proof. For $j<\left(N_{k+1} / N_{k}\right)^{2}\left(A_{k+1}+M\right)$, the conclusion follows from Claim 7.3. If $j \geq\left(N_{k+1} / N_{k}\right)^{2}\left(A_{k+1}+M\right)$, then there exist $k^{\prime}>k$ and $z^{\prime} \in \mathcal{L}_{N_{k^{\prime}}}$ such that $z^{\prime} \in U_{k^{\prime}}\left(j^{\prime}\right)$ with

$$
A_{k^{\prime}}+M \leq j^{\prime} \leq\left(\frac{N_{k^{\prime}+1}}{N_{k}}\right)^{2}\left(A_{k^{\prime}+1}+M\right)
$$

and $\mathcal{P}\left(z, N_{k}\right) \subseteq \mathcal{P}\left(z^{\prime}, N_{k^{\prime}}\right)$. Then by Claim 7.3 applied to $k^{\prime}$ we get

$$
\begin{aligned}
\int_{[-1,1]^{d-1}} & P_{\omega}\left(\left\{Y_{n}\right\} \cap \mathcal{P}\left(z, N_{k}\right) \neq \emptyset \mid W^{(w)}\right) d w \\
\leq & \int_{[-1,1]^{d-1}} P_{\omega}\left(\left\{Y_{n}\right\} \cap \mathcal{P}\left(z^{\prime}, N_{k^{\prime}}\right) \neq \emptyset \mid W^{(w)}\right) d w \leq(\log u)^{(1-d)(1-2 \epsilon)} .
\end{aligned}
$$




\subsection{Expected number of bad blocks that are visited}

Fix $k$. Let

$$
\begin{aligned}
& \mathcal{D}(k)=\left\{z \in \mathcal{L}_{k} \cap B_{2 L} \mid \mathcal{P}\left(z, N_{k}\right) \text { is not good }\right\}, \\
& \mathcal{B}(k)=\#\left\{z \in \mathcal{D}(k) \mid\left\{Y_{\ell}\right\} \cap \mathcal{P}\left(z, N_{k}\right) \neq \emptyset\right\} .
\end{aligned}
$$

We are interested in the distribution of the variable $\mathcal{B}(k)$.

Lemma 7.5. Fix $k$ and $\omega \in G$. Then

$$
\int_{[-1,1]^{d-1}} E_{\omega}\left(\mathcal{B}(k) \mid W^{(w)}\right) d w \leq 3(\log u)^{1-\epsilon} .
$$

Proof. Let

$$
\begin{aligned}
& \mathcal{D}^{(1)}(k)=\mathcal{D}(k) \cap\left\{z:\left\langle z, e_{1}\right\rangle \leq N_{k}^{2}\left(A_{k}+M\right)\right\}, \\
& \mathcal{D}^{(2)}(k)=\mathcal{D}(k) \cap\left\{z:\left\langle z, e_{1}\right\rangle>N_{k}^{2}\left(A_{k}+M\right)\right\},
\end{aligned}
$$

and for $i=1,2$ let

$$
\mathcal{B}^{(i)}(k)=\left|\left\{z \in \mathcal{D}^{(i)}(k):\left\{Y_{\ell}\right\} \cap \mathcal{P}\left(z, N_{k}\right) \neq \emptyset\right\}\right| .
$$

Then $\left\{Y_{\ell}\right\}$ visits no more than $A_{k}+M$ elements of $\mathcal{D}^{(1)}(k)$, and thus $\mathcal{B}^{(1)}(k) \leq A_{k}+M \leq$ $2(\log u)^{1-\epsilon}$

Let $z \in \mathcal{D}^{(2)}(k)$. Then by Lemma 7.4,

$$
\int_{[-1,1]^{d-1}} P_{\omega}\left(\left\{Y_{n}\right\} \cap \mathcal{P}\left(z, N_{k}\right) \neq \emptyset \mid W^{(w)}\right) d w \leq(\log u)^{(1-d)(1-2 \epsilon)} .
$$

Therefore, using (5.7) and (5.4), we get

$$
\begin{aligned}
\int_{[-1,1]^{d-1}} E_{\omega}\left(\mathcal{B}^{(2)}(k) \mid W^{(w)}\right) d w & \leq(\log u)^{\alpha+\epsilon+(1-d)(1-2 \epsilon)} \\
& =(\log u)^{\alpha-d+1+(2 d-1) \epsilon} \leq(\log u)^{1-\epsilon} .
\end{aligned}
$$

Altogether, we get

$$
\begin{aligned}
\int_{[-1,1]^{d-1}} E_{\omega}\left(\mathcal{B}(k) \mid W^{(w)}\right) d w \leq & \int_{[-1,1]^{d-1}} E_{\omega}\left(\mathcal{B}^{(1)}(k) \mid W^{(w)}\right) d w \\
& +\int_{[-1,1]^{d-1}} E_{\omega}\left(\mathcal{B}^{(2)}(k) \mid W^{(w)}\right) d w \\
\leq & 3(\log u)^{1-\epsilon} .
\end{aligned}
$$

\section{Proof of main result}

In this section we prove Theorem 1.5 . 
Proof of Theorem 1.5. By Lemma 7.5,

$$
\int_{[-1,1]^{d-1}} E_{\omega}\left(\sum_{k=1}^{\iota} \mathcal{B}(k) \mid W^{(w)}\right) d w \leq 3 \iota(\log u)^{1-\epsilon} .
$$

Therefore, there exists $w$ such that

$$
E_{\omega}\left(\sum_{k=1}^{\iota} \mathcal{B}(k) \mid W^{(w)}\right) \leq 3 \iota(\log u)^{1-\epsilon} .
$$

We now fix $w$ to be such a value. Let

$$
\bar{W}=W^{(w)} \cap\left\{\sum_{k=1}^{\iota} \mathcal{B}(k) \leq 6 \iota(\log u)^{1-\epsilon}\right\} .
$$

Then by Markov's inequality, $P_{\omega}(\bar{W}) \geq 0.5 P_{\omega}\left(W^{(w)}\right) \geq \frac{1}{2} u^{\epsilon-1 / 2}$. Note that there is a set $V$ of paths such that

$$
\bar{W}=\left\{\left\{Y_{n}\right\} \in V\right\},
$$

and for every $v \in V$, by Lemma 6.5 and by (8.1) and the choice of $\chi$ and $\psi((5.5),(5.6))$,

$$
\frac{P_{\omega}\left(X_{j}=v_{j} \text { for all } j<N_{v}\right)}{P_{\omega}\left(Y_{j}=v_{j} \text { for all } j<N_{v}\right)} \geq \frac{1}{2} \eta^{\left(l+2+6 \iota(\log u)^{1-\epsilon}\right) L^{3 x+4 \psi}} \geq u^{\epsilon-1 / 2} .
$$

Therefore,

$$
P_{\omega}\left(\left\{X_{n}\right\} \in V\right) \geq u^{\epsilon-1 / 2} P_{\omega}\left(\left\{Y_{n}\right\} \in V\right) \geq u^{\epsilon-1} .
$$

Every path in $V$ reaches $\partial^{+} B_{2 L}$ before returning to 0 , and therefore we get (5.3), from which we deduce Proposition 2.2 and Theorem 1.5.

Acknowledgments. I wish to thank A.-S. Sznitman for introducing this problem to me, and to thank G. Kozma, T. Schmitz and O. Zeitouni for useful discussions. In addition I thank O. Zeitouni for suggesting that I use the methods of [6] in order to prove Corollary 1.6. A very careful and detailed referee report contributed significantly to the quality of the presentation, and I am grateful for that.

The research was partially supported by grant 2006477 of the Israel-U.S. Binational Science

Foundation, by grant 152/2007 of the German Israeli foundation and by ERC StG grant 239990.

\section{References}

[1] Berger, N., Zeitouni, O.: A quenched invariance principle for certain ballistic random walks in i.i.d. environments In: In and Out of Equilibrium 2, V. Sidoravicius and M. E. Vares (eds.), Progr. Probab. 60, Birkhäuser, 137-160 (2008) Z Zbl 1173.82324 MR 2477380

[2] Bolthausen, E., Sznitman, A.-S.: On the static and dynamic points of view for certain random walks in random environment. Methods Appl. Anal. 9, 345-375 (2002) Zbl 1079.60079 MR 2023130

[3] Dembo, A., Peres, Y., Zeitouni, O.: Tail estimates for one-dimensional random walk in random environment. Comm. Math. Phys. 181, 667-683 (1996) Zbl 0868.60058 MR 1414305 
[4] Drewitz, A., Ramírez, A. F.: Ballisticity conditions for random walk in random environment. Probab. Theory Related Fields 150, 61-75 (2011) Zbl pre05950525

[5] Durrett, R.: Probability: Theory and Examples. Duxbury Press (1996) MR 1609153

[6] Gantert, N., Zeitouni, O.: Quenched sub-exponential tail estimates for one-dimensional random walk in random environment. Comm. Math. Phys. 194, 177-190 (1998) Zbl 0982.60037 MR 1628294

[7] Peterson, J.: Limiting distributions and large deviations for random walks in random environments. PhD Thesis, Univ. of Minnesota (2008) MR 2711962

[8] Sznitman, A.-S.: Slowdown estimates and central limit theorem for random walks in random environment. J. Eur. Math. Soc. 2, 93-143 (2000) Zbl 0976.60097 MR 1763302

[9] Sznitman, A.-S.: On a class of transient random walks in random environment. Ann. Probab. 29, 724-765 (2001) Zbl 1017.60106 MR 1849176

[10] Sznitman, A.-S.: An effective criterion for ballistic behavior of random walks in random environment. Probab. Theory Related Fields 122, 509-544 (2002) Zbl 0995.60097 MR 1902189

[11] Sznitman, A.-S., Zerner, M.: A law of large numbers for random walks in random environment. Ann. Probab. 27, 1851-1869 (1999) Zbl 0965.60100 MR 1742891

[12] Varadhan, S. R. S.: Large deviations for random walks in a random environment. Comm. Pure Appl. Math. 56, 1222-1245 (2003) Zbl 1042.60071 MR 1989232

[13] Yilmaz, A.: Averaged large deviations for random walk in a random environment. Ann. Inst. H. Poincaré Probab. Statist. 46, 853-868 (2010) Z Zbl 1201.60098 MR 2682269

[14] Zerner, M. P. W.: A non-ballistic law of large numbers for random walks in i.i.d. random environment. Electron. Comm. Probab. 7, 191-197 (2002) Zbl 1008.60107 MR 1937904 